\title{
The Role of Hydromechanical Coupling in Fractured Rock Engineering
}

\section{Jonny Rutqvist $^{1}$ and Ove Stephansson ${ }^{2}$}

1. Lawrence Berkeley National Laboratory, Earth Sciences Division, MS 90-1116, Berkeley, CA 947 05, Phone \#+1-(510)-486-5432, Fax \#+1-(510)-486-5686, E-mail: jrutqvist@lbl.gov

2. Royal Institute of Technology, Department of Land and Water Resources Engineering, SE-100 44 Stockholm, Sweden and GeoForschungsZentrum, D-14473 Potsdam, Germany

Dec 1, 2002

Hydrogeology Journal (in press) 


\begin{abstract}
This paper provides a review of hydromechanical (HM) couplings in fractured rock, with special emphasis on HM interactions as a result of or directly connected with human activities. In the early 1960s, the coupling between hydraulic and mechanical processes in fractured rock started to receive wide attention. A series of events-including dam failures, landslides, and injection-induced earthquakes — were believed to result from HM interaction. Moreover, the advent of the computer technology in the 1970s made possible the integration of nonlinear processes such as stress-permeability coupling and rock mass failure into coupled HM analysis. Coupled HM analysis is currently being applied to many geological engineering practices. One key parameter in such analysis is a good estimate of the relationship between stress and permeability. Based on available laboratory and field data it was found that the permeability of fractured rock masses tends to be most sensitive to stress changes at shallow depth (low stress) and in areas of low in situ permeability. In highly permeable fractured rock sections, fluid flow may take place in clusters of connected fractures that are locked open as a result of previous shear dislocation or partial cementation of hard mineral filling. Such locked open fractures tend to be relatively insensitive to stress and may therefore be conductive at great depths. Because of out the great variability of HM properties in fractured rock, and the difficulties in using laboratory data for deriving in situ material properties, the HM properties of fractured rock masses are best characterized in situ.
\end{abstract}

\title{
Keywords:
}

fractured rocks, mechanical, hydromechanical coupling, stress, permeability 


\section{INTRODUCTION}

In earth sciences, the term "hydromechanical (HM) coupling" refers to the physical interaction between hydraulic and mechanical processes. HM interactions are common in geological media (e.g., soils and rocks) because such media contain pores and fractures, which can be fluid filled and deformable. In general, a fluid-saturated porous medium or rock fracture can deform either as a result of change in the external load or change in the internal pore-fluid pressure (Figure 1). An increased compressive external load (or stress) means that the porous medium as a whole will be compressed to both a smaller bulk volume and a smaller pore volume. If the external load is applied "rapidly", the reduced pore volume will tend to compress pore fluid and thereby increase pore fluid pressure, because the fluid has no time to escape. This is the so-called "undrained HM response". If, on the other hand, the external load is applied "slowly", the fluid has time to escape the compressing volume, in which case, the fluid pressure shows almost no increase at all. This is the so-called "drained HM response". Likewise, a reduction in fluid pressure or fluid mass can cause a settlement of the porous media, with accompanying reduction in bulk and pore volume.

In general, the HM couplings discussed above can be described as "direct" HM couplings, or couplings that occur through deformation and pore-fluid interactions. Direct HM couplings include two basic phenomena (Wang 2000):

(i) A solid-to-fluid coupling that occurs when change in applied stress produces a change in fluid pressure or fluid mass. 
(ii) A fluid-to-solid coupling that occurs when a change in fluid pressure or fluid mass produces a change in the volume of the porous medium.

These two cases of direct HM couplings are shown schematically in Figure 2 and are labeled (i) and (ii). In any of these two cases, the reduction in pore volume leads to a reduction of the cross-sectional area and a reduction in fluid flow capacity. Furthermore, reduction of pore volume may result in a stiffer material, as more contacts are created between neighboring grains. These changes imply that mechanical and hydraulic processes can affect each other through changes in material properties, which can be considered "indirect" HM couplings. Accordingly, two basic phenomena of indirect HM coupling may be considered:

(iii) A solid-to-fluid coupling that occurs when an applied stress produces a change in hydraulic properties

(iv) A fluid-to-solid coupling that occurs when a change in fluid pressure produces a change in mechanical properties

These phenomena are labeled (iii) and (iv) in Figure 2. Both direct and indirect coupled processes may be fully reversible. However, inelastic responses, including yielding, fracturing, or fault slip, cause irreversible changes in porous and fractured media. While direct HM couplings occur in all types of geological media, they tend to be most important in relatively soft and low-permeability rocks and soils. Indirect HM couplings tend to be most important in fractured rock or intact rock with flat inter-grain micropores, where changes in permeability caused by fracture or pore dilation can be dramatic. 
This paper focuses on coupled HM processes triggered by human activities (such as underground injection and underground construction), leaving out those induced by natural geological processes, which are thoroughly reviewed in an accompanying article of this special issue (Neuzil 2003). This paper is focused on the deeper water-saturated zone of the bedrock, leaving out issues related to HM behavior in shallow soils and clays, which are also treated in an accompanying paper in this issue (Alonso et al. 2003). Furthermore, this paper is focused on indirect HM coupling, especially the coupling between stress and permeability, which is most relevant in fractured rock. The presentation is structured in the sequence of observations, analysis, and applications. After providing a brief background in Section 2, the basic HM behavior of porous intact rock and fractured porous rock derived from laboratory experiments and theoretical studies is introduced in Section 3. Thereafter, in situ HM behavior of fractured rock masses is introduced. This includes current HM conditions in fractured rock masses (Section 4) and observations of in situ coupled HM interactions triggered by human activities in (Section 5). In Section 6, modeling of HM coupling is briefly reviewed. Finally, Section 7 presents current applications of HM analysis in a number of engineering practices.

\section{BACKGROUND}

Direct HM interactions in the earth's crust have been recognized since the late 1800s (Wang 2000). The earliest observations included the response of water levels in wells to ocean tidal loading and passing trains (solid-to-fluid couplings), as well as subsidence of the land surface caused by extraction of water, oil, and gas (a fluid-to-solid coupling). 
Also, Meinzer (1928) observed large yields of groundwater from the Dakota Sandstone, which could not be explained without the extra storage provided by HM changes in pore volume. Observations such as these lead to Terzaghi's (1923) classical contributions to the HM field, for problems related to settlement analysis, and Theis (1935) analysis of pumped wells.

Terzaghi defined effective (intergranular) stress, $\sigma_{z z}^{\prime}$, in a geological medium, as the total vertical stress, $\sigma_{z z}$, less the pore fluid pressure, $p$ :

$$
\sigma_{z z}^{\prime}=\sigma_{z z}-p
$$

Equation (1) is the most fundamental equation for describing coupling between hydraulic and mechanical processes in geological media. Terzaghi's effective stress law was initially applied to problems related to settlement analysis, dam and slope stability, and petroleum technology for well stimulation. Terzaghi also derived the first coupled equation for consolidation, which is the diffusion equation for excess (greater than hydrostatic) water pressure $p_{e x}$ :

$$
\frac{\partial p_{e x}}{\partial t}=c \frac{\partial^{2} p_{e x}}{\partial z^{2}}
$$

where $\mathrm{c}$ is a type of diffusivity term known as the consolidation coefficient.

Jacob (1940) developed the Theis concept of aquifer storage into a mathematical definition for the coefficient of storage or storativity, $S$ :

$$
S=\frac{1}{\rho_{f} A} \frac{\Delta m_{f}}{\Delta h}
$$


where $\rho_{f}$ is the density of water, $A$ is the horizontal cross-sectional area for a vertical column of aquifer, $\Delta m_{f}$ is the change in water mass in the column, and $\Delta h$ is the change in head. Assuming incompressible grains in a compressible porous media, Jacob (1940) derived the expression for $S$ of the type

$$
S=\rho_{f} g H\left(\frac{\phi}{K_{f}}+\frac{1}{K^{\prime}}\right)
$$

where $g$ is gravity, $\phi$ is porosity, $H$ is aquifer thickness, $K_{f}$ is the bulk modulus of pore fluid, and $K^{\prime}$ is the vertical compressibility of the aquifer. Jacob's diffusion equation for two-dimensional (in the plane of the aquifer) transient flow in a confined aquifer is:

$$
\frac{\partial p}{\partial t}=\frac{T}{S}\left(\frac{\partial^{2} p}{\partial x^{2}}+\frac{\partial^{2} p}{\partial y^{2}}\right)
$$

where $T$ is transmissivity and $t$ is time. Jacob (1950) derived the three-dimensional diffusion equation,

$$
\frac{\partial h}{\partial t}=\frac{\rho_{f} g k}{\mu_{f} S_{s}} \nabla^{2} h
$$

where $h$ is hydraulic head, which is the elevation head plus the pressure head (that is $h=z$ $+p / \rho_{f} g, \mu_{f}$ is fluid viscosity, $k$ is permeability, and $S_{s}$ is specific storage defined as:

$$
S_{s}=\rho_{f} g\left(\frac{\phi}{K_{f}}+\frac{1}{K^{\prime}}\right)
$$

Jacob's diffusion equations (e.g., Equations (5) and (6)) are "partially coupled" in the sense that two- or three-dimensional flow is coupled to a simplified one-dimensional mechanical model of vertical deformation. This is the basic concept behind most ground water flow equations and well-test solutions derived in geohydrology. 
Biot (1941) extended Terzaghi's concept of one-dimensional consolidation to a general theory of three-dimensional consolidation. Biot's equations for isotropic linear elastic porous media can be written in a "mixed stiffness form"(Wang 2000), as

$$
\begin{aligned}
& \sigma_{m}=K \varepsilon_{v}+\alpha p \\
& \xi=-\alpha \varepsilon_{v}+\frac{1}{M} p
\end{aligned}
$$

where $\sigma_{m}$ is the total mean stress (positive for compression), $K$ is the usual (drained) bulk modulus (Figure $1 b$ ), $\varepsilon_{v}$ is the volumetric strain (positive for contraction), $\alpha$ is the BiotWillis' coefficient (Biot and Willis (1957)), $\xi$ is the increment of fluid content (positive for "gain" of fluid), and $M$ is Biot's modulus. Equation (8) governs the elastic responses of the pore structure; Equation (9) governs pore fluid responses. The two equations are coupled through the volumetric strain and fluid pressure terms. Since the theory describes interaction between pore fluid and elastic responses, it has been called the theory of poroelasticity. The introduction of the Biot-Willis coefficient as a factor multiplied to fluid pressure in Equation (8) signifies a modification and generalization of Terzaghi's effective stress law to:

$$
\sigma_{m}^{\prime}=\sigma_{m}-\alpha p
$$

The coefficient $\alpha$, which usually ranges between 0 and 1 , has been measured in laboratory experiments (e.g., Nur and Byerlee 1971) for a range of geological materials. For soils, this coefficient is generally close to one, whereas in rocks it can be significantly less than one. 
The original Biot (1941) formulation of linear poroelasticity was subsequently reexamined several times by Biot $(1955,1956)$ himself, by Geertsma (1966) for settlement analysis, by Verruijt (1969) for aquifer behavior; and by Rice and Cleary (1976), for redefining the material constants. The theory has also been extended to more sophisticated material models and to incremental variations in nonlinear systems. However, the pioneering work by Terzaghi, Theis, and Biot form the basic framework for modern coupled HM analysis in geological media currently applied in many disciplines of earth sciences.

Human-triggered indirect HM interactions in the earth's crust were probably first recognized in the 1920s and 1930s, when seismic tremors were felt near oil-producing fields (Pratt and Johnson 1926) and water reservoirs (Carder 1945). These HM interactions, known as "induced seismicity," were the results of pressure induced irreversible failures, usually in the form of shear slip along discontinuities. The most fundamental criterion for fault slip is derived from the effective stress law and a Coulomb criterion, rewritten as:

$$
\sigma_{s c}=\sigma_{s c 0}+\mu_{s}\left(\sigma_{n}-p\right)
$$

where $\sigma_{s c}$ is the critical shear stress, $\sigma_{s c 0}$ is cohesion, $\mu_{s}$ is coefficient of shear friction, and $\sigma_{n}$ is the normal stress (Scholz 1990). This equation was also applied in the early 1900s to various engineering applications in soil mechanics, dam and slope stability, and petroleum technology. 
Intentional hydraulic fracturing was introduced into petroleum industry in the late 1940s to increase production through well stimulation (Clark 1949). The most fundamental criterion for tensile failure (or hydraulic fracturing) is that incipient fracture propagation will occur when the fluid pressure exceeds the least principal stress by an amount equal to or greater than the tensile strength of the rock:

$$
p_{c t} \geq \sigma+\sigma_{t}
$$

Hydraulic-fracturing theory, as described by Hubbert and Willis (1957), was suggested as a method to determine in situ stress in the 1960s (Fairhurst 1964). In classical hydraulicfracturing stress-measurement theory, the breakdown of the borehole wall and fracture initiation takes place when the least compressive tangential effective stress is equal to the tensile stress of the rock (Haimson and Fairhurst 1967). In parallel with the development of techniques for stress measurement, the technology for propagation and control of hydraulic fractures was developed in petroleum engineering (Perkins and Kern 1961; Geertsma and Deklerk 1969).

In the early 1960s, HM interactions in fractured rock got wider attention. A series of events—including dam failures, landslides, and injection-induced earthquakes—were suggested to be triggered by HM interaction. These events called for improved HM analysis, especially regarding dam stability, which at that time did not include special treatment of fissured rocks and rock fractures. As a result, some of the earliest works on coupled HM behavior in fractured rocks were related to dam foundations (Londe and Sabarly 1966; Louis and Maini 1970). This included application of the parallel plate flow concept to describe fracture flow (Snow 1965; Louis and Maini 1970), which is given by 


$$
\frac{Q^{f}}{\nabla h}=\frac{b^{3} \rho_{f} g}{w 12 \mu_{f}}
$$

where $Q^{f}$ is volume flow rate per unit plate (or fracture) width $(w), \nabla h$ is head gradient, and $b$ is the fracture aperture or the physical separation between two smooth parallel plates. In rock mechanics, the concept of "deformable" fractures was introduced, where fractures can respond to changes in effective normal stress and shear stress in an elastic manner, even if no failure takes place (Figure le and $f$ ). This is formulated according to Goodman (1970) as:

$$
\begin{aligned}
& \Delta u_{n}=k_{n} \Delta \sigma_{n}^{\prime} \\
& \Delta u_{s}=k_{s} \Delta \sigma_{s}
\end{aligned}
$$

In Equation (14) $\Delta u_{n}$ is the normal deformation of the fracture caused by a change in effective normal stress, $\Delta \sigma_{n}^{\prime}$, with the magnitude of opening or closure depending on the fracture normal stiffness $k_{n}$ (Figure 1e). Likewise, Equation (15) describes the shear displacement, $\Delta u_{s}$, which depends on the shear stiffness, $k_{s}$, and the change in shear stress, $\Delta \sigma_{s}$ (Figure 1f). Since pioneering works by Snow (1965), Louis and Maini (1970), and Goodman (1970), the coupled HM behavior of rock fractures has continued to be a hot topic, both in theoretical studies and practical applications, including the development of more sophisticated constitutive models. The empirical work on constitutive models by Barton and Bandis (Barton and Choubey 1977; Bandis et al. 1983; Barton et al. 1985) has been especially important for practical applications.

Since digital computer become widely available, in the 1970s, much development of HM coupling in fractured rock has been related to improving numerical modeling and its 
application to complex geological systems. Sandhu and Wilson (1969), Noorishad (1971), Ghaboussi and Wilson (1973), and Gambolati and Freeze (1973) were among the first to use numerical modeling for coupled HM analysis. These early finite-element models treated either linear hydro-elastic phenomena in porous media with fully implicit coupling (e.g. Sandhu and Wilson 1969) or discrete-fractured media using sequential explicit coupling between the hydraulic and mechanical analysis (e.g., Noorishad 1971). For fractured and porous media, fully coupled HM numerical models have been available since the early 1980s, when Noorishad et al. (1982) presented a coupled HM formulation and finite-element scheme that later evolved into the computer code ROCMAS. This formulation was based on an extension of Biot's theory of consolidation (Biot 1941) to include discrete fractures in addition to the porous matrix, and uses a fully implicit solution technique. Since then, many computer codes capable of modeling coupled HM processes in fractured porous media have been developed using various numerical methods, including finite-element, distinct-element and boundary-element methods (Jing and Hudson 2002). At the same time, more realistic constitutive models have been developed to describe coupled HM interaction in rock fractures, with ones by Barton et al. 1985 and Walsh (1981) most commonly applied. Efforts have also been made to incorporate HM coupling into effective medium theories (e.g. Oda (1982)). As a result of these developments, direct and indirect HM interactions have been integrated into coupled HM numerical analysis of complex geological media, which includes material heterogeneity, complex geometry, and material nonlinearity. 
During the last 20 years, most of the research and development on HM coupling in fractured rocks and most applications of HM coupled analysis have been conducted as part of oil and gas exploration, hot-dry rock geothermal energy investigations, and studies for nuclear waste disposal. Relatively new applications of HM coupling include deep injection of solid waste and geological sequestration of greenhouse gases. The results of these research efforts and some applications of coupled HM analysis will be discussed in more detail below.

\section{FUNDAMENTALS OF HM BEHAVIOR IN INTACT ROCK AND ROCK FRACTURES}

Rock masses can be considered to be composed of intact (unfractured) rock matrix and rock fractures. Although intact rock has fractures in the form of microcracks, which might be similar in behavior to macrofractures, it is still useful to study the fundamentals of intact rock and rock fractures separately. This section presents fundamental HM behavior of intact rock and rock fractures as has been derived from controlled laboratory and field tests.

\section{Fundamental HM behavior of intact rock}

The fundamental macroscopic HM behavior of intact rock can be described in terms of measurable macroscopic quantities for porous media (as a whole). Macroscopic properties for hydromechanical behavior of intact rock include Biot's $\alpha$, Biot's modulus $M$, and the relationship between stress (or strain) and permeability. Most published HM experiments have been conducted to determine Biot's $\alpha$ (e.g. Nur and Byerlee (1971), 
and Bernabe (1986)) or permeability variation with both confining pressure and fluid pressure (e.g., Brace et al. (1968); Bernabe (1986); Neuzil (1986); Kilmer et al. (1987)). A recent review of experiments on intact rock is presented in this Special Issue by Heiland (2003).

Figure 3 presents literature data of permeability versus effective confining stress from laboratory tests on shale, granite and low permeability sandstone. Shale, which has the lowest permeability, also has the most stress-sensitive permeability. The tight gas sandstone is very stress sensitive at low stress, but appears to attain a residual permeability at higher stress.

The differences in the stress-permeability relationship for different rock types in Figure 3 can be explained by differences in pore shapes. The effect of pore shape can be studied, treating pores as elliptical cracks in a linear elastic medium, according to the following equation:

$$
b_{c}=b_{c 0}\left[1-\frac{2\left(1-v^{2}\right)}{R E} P\right]
$$

where $b_{c 0}$ is the crack's aperture under zero pressure, $v$ is Poisson's ratio, $E$ is Young's modulus, $R$ is aspect ratio, and $P$ is confining pressure, meaning an isotropic compressive stress (Iwano 1995; Walsh 1965). This equation implies that cracks will be completely closed when the pressure reaches

$$
P=\frac{R E}{2\left(1-v^{2}\right)}
$$


Equation (17) shows that cracks subjected to a stress $P$ will close more easily if they are flat, (i.e., if they have a small aspect ratio, $R$ ). Cracks with small aspect ratios are generally found in less permeable rocks, such as shale and granite, which explains their relatively sensitive stress-permeability relationship. Also, in low-permeability gas sand, the basic cause of unusually stress sensitive permeability at low stress has been ascribed to high-aspect-ratio sheet pores that are commonly observed between grain boundaries (Brower and Morrow 1985). Isotropic pores in the sandstone that are more resistant to stress can explain the relatively insensitive permeability in these media at high stress.

Quite a few empirical models have been used to match observed permeability, $k$ versus confining pressure, $P$, and fluid pressure, $p$, in intact rock. Among them exponential functions, (e.g., Louis et al. (1977)):

$$
k=k_{o} \exp (-a l(P-p))
$$

power functions, (e.g., Kranz et al. (1979)):

$$
k=k_{o}[P-p]^{-a 2}
$$

and the logarithmic function proposed by Jones and Ovens (1980):

$$
\log k=-a 3 \log (P-p)+a 4 \text {. }
$$

In Equations $(18)-(20) k_{o}$ is permeability at some reference effective stress, which is 0 in the case of Equation (18), and $a 1, a 2, a 3$, and $a 4$ various fitting parameters to match experimental data. 
Triaxial tests are frequently used in petroleum engineering to simulate reservoir conditions and to record porosity values versus vertical effective stress (Van-Golf Racht 1982). A stress-permeability relation obtained from triaxial tests might be combined with a permeability-porosity relation to obtain the stress-porosity relationship (e.g., Davies and Davies 1999). A permeability-porosity relationship is provided by the theoretical Carman-Kozeny relationship (Scheidegger 1974), which for the case of packed uniform spheres can be written as

$$
k=\frac{d_{g}{ }^{2} \phi^{3}}{72 \tau_{C K}(1-\phi)^{2}}
$$

where $d_{g}$ is the diameter of spheres and $\tau_{C K}$ is tortuosity. However, empirical relationships of the type

$$
k=e^{a 5 \phi+a 6}
$$

are widely used to match experimental data where $a 5$ and $a 6$ are fitting constants (VanGolf Racht 1982).

The macroscopic Biot-Willis' coefficient, $\alpha$, can also be expressed in terms of grain and bulk properties:

$$
\alpha=1-\frac{K}{K_{s}}
$$

where $K$ is the drained bulk modulus of the medium and $K_{s}$ is the bulk modulus of the solid grains. Thus $\alpha \approx 1$ for a medium in which rock grains have a large bulk modulus (very stiff) compared with bulk modulus for the medium as a whole. Similarly, the inverse of Biot's modulus, $M$, can be expressed in micromechanical parameters as 


$$
\frac{1}{M}=\frac{\phi}{K_{f}}+\frac{\alpha-\phi}{K_{s}}
$$

Equation (24) is valid for an ideal porous medium characterized as a fully connected pore space in a microscopically homogenous and isotropic matrix (Detournay and Cheng 1993). Equation (24) indicates that $1 / M$ varies with effective stress, since $\phi$ and $\alpha$ both vary with stress. Wang (2000) lists values of $M$ and other poroelastic constants for various rock types.

Some laboratory experiments have been carried out to study permeability changes caused by deviatoric stresses (e.g., Zoback and Byerlee 1975; Wang and Park 2002). In these tests, the axial load on the core sample is increased, and the axial permeability is measured as a function of differential stress (axial stress less confining stress). Test results on granite by Takahashi et al. (1995), Lee and Chang (1995), and Souley et al. (2001) show that the permeability first decreases about one order of magnitude, until the axial stress has reached about half of the maximum stress (stress at rock failure). This reduction in permeability results from closure of existing pores and microfractures. At higher stress, that is, values over half of the rock strength, the permeability increases, with increasing axial stress causing the onset of unstable crack growth. The permeability increase near the peak stress can be dramatic and is related to macroscopic failure by the coalescence of microcracks (Souley et al. 2001). 


\section{Fundamental HM behavior of rock fractures}

In a fracture, fluid flow takes place in the void between two rock surfaces, that are irregularly shaped and partly in contact with each other. The HM behavior of rock fractures has been studied in experiments using various techniques, such as uniaxial normal compression tests, triaxial cell tests and various bi-axial compression/shear tests. Most experiments have been conducted to study HM behavior under normal closure; only a few have been conducted to study the effects of shear on permeability.

\section{Hydraulic fluid flow}

Hydraulic behavior in a rock fracture can be modeled analogously to that in a confined aquifer using a diffusion equation of the form

$$
\frac{\partial h}{\partial t}=\frac{T^{f}}{S^{f}} \nabla h
$$

where $T^{f}$ and $S^{f}$ are fracture transmissivity and storativity, respectively. A hydraulic aperture, $b_{h}$, can be defined as the parallel plate fracture aperture $b$ in Equation (13) that produces the same relationship between $Q$ and $\Delta h$. Hence, fracture transmissivity is given by

$$
T=\frac{b_{h}^{3} \rho_{f} g}{12 \mu_{f}}
$$

The hydraulic or effective aperture $b_{h}$ can therefore be back-calculated from fracture transmissivity, which can be determined in a flow test. In analogy with Jacob's equations for storage in a confined aquifer (Equations (3) and (4)), the coefficient of storage (or storativity) of a rock fracture can be estimated as (Rutqvist et al. 1998): 


$$
\begin{aligned}
& S=\frac{1}{\rho_{f} A} \frac{\Delta m_{f}}{\Delta h}=\frac{1}{A} \frac{\Delta\left(\rho_{f} g V_{f}\right)}{\Delta P} \\
& S=\rho_{f} g b\left(\frac{\phi}{K_{f}}+\frac{1}{K^{\prime}}\right)=\rho_{f} g\left(\frac{b_{v}}{K_{f}}+\frac{1}{k_{n}}\right)
\end{aligned}
$$

where $b_{v}$ is the void aperture defined as the volume accessible for water per unit area of the fracture, which is equal to the average physical fracture aperture $\bar{b}$ over $A$.

\section{Mechanical normal closure behavior}

When normal stress is applied on joints, the normal deformation is typically nonlinear as shown in Figure 4a. The rate of deformation is greatest at low values of normal stress, indicating that fracture stiffness increases as normal stress increases. A common feature of fracture deformation is a hysteresis effect during stress loading and unloading (not included in Figure 4a), which is caused by processes arising from surface mismatch, sampling disturbances and crushing of asperities (Barton et al. 1985). In Figure 4a, a size effect on fracture normal closure is indicated by the experimental results of Yoshinaka et al. (1993), which showed that the maximum closure, $\delta_{\max }$, increases with sample size. Several empirical models have been developed and applied for normal closure behavior. The first nonlinear joint model, is Goodman's (1974) hyperbolic form, which can be written as:

$$
\Delta u_{n}=\frac{\sigma_{n i}^{\prime}}{k_{n i}}\left(1-\frac{\sigma_{n i}^{\prime}}{\sigma_{n}^{\prime}}\right)
$$


where $\Delta u_{n}$ is joint normal displacement, and $k_{n i}$ and $\sigma_{n i}^{\prime}$ are normal stiffness and effective normal stress at an initial reference stage (Figure 4a). However, the most commonly applied joint model today is Bandis' hyperbolic function (Bandis et al. 1983):

$$
\delta=\frac{\sigma_{n}^{\prime}}{k_{n 0}+\sigma_{n}^{\prime} / \delta_{\max }}
$$

where $\delta$ is current normal closure, $\delta_{\max }$ is maximum normal closure, and $k_{n 0}$ is normal stiffness at the zero stress intercept (Figure $4 a$ ). The basic parameters $k_{n 0}$ and $\delta_{\max }$ can be estimated from Barton-Bandis basic joint parameters; Joint Roughness Coefficient $\left(J R C_{0}\right)$, and Joint Compressive Strength $\left(J C S_{0}\right)$ using empirical relationships (Barton et al. 1985).

The second most commonly used model is the logarithmic type, which has mostly been applied in hot-dry rock geothermal-reservoir engineering. In one version, the logarithmic model can be written as (Evans et al. 1992):

$$
\Delta u_{n}=-\left(d k_{n} / d \sigma_{n}^{\prime}\right)^{-1} \ln \frac{\sigma_{n}^{\prime}}{\sigma_{n 0}^{\prime}}
$$

The parameter $\left(d k_{n} / d \sigma_{n}^{\prime}\right)^{-1}$, which Evans et al. (1992) denoted "stiffness characteristics," can be estimated theoretically from Hertz's analysis of deformation for two fracture surfaces containing linear elastic hemispheres in contact.

Both of these models are attractive, because their behavior can be estimated from basic fracture surface data and they have been matched to a large number of laboratory data in the literature. In general, the hyperbolic equation (30) has been shown to match mated 
fractures better whereas the logarithmic equation (31) matches unmated fractures better. However, as shown by Wei and Hudson (1988), Evans et al. (1992), and Zhao and Brown (1992), the logarithmic function can also match laboratory data of mated fractures over an engineering stress range (i.e., less than about $10 \mathrm{MPa}$ ). At very high stress, on the other hand, the logarithmic model cannot be used to produce a residual aperture, since the fracture will close completely and ultimately produce a negative aperture.

\section{Mechanical shear behavior}

Mechanics of fracture shear behavior have been developed in the field of rock mechanics since the 1960s, pioneered by Patton (1966), who predicted the existence of scale effects. The influence of scale effects was quantified by Barton and Choubey (1977), and conclusive experiments were presented by Bandis et al. (1983). Figure $4 c$ illustrates a typical shear stress displacement of a clean, rough, dilatant fracture under constant normal stress. It is characterized by rapid increase in shear stress up to a peak, followed by a loss in load-carrying capacity. The shear displacement is accompanied with a shear dilation, as shown in the lower curves of Figure $4 c$. For a portion of the stress/deformation curve corresponding to elastic deformation of the fracture, there is minimum dilation. The onset of rapid dilation occurs when asperities begin to slide against each other. Rate of dilation (slope of $\Delta u_{n}$ curve in Figure 4c) increases and reaches a maximum at the peak shear stress (Barton et al. 1985). The figure also illustrates the effect of scale. For a larger sample, the peak shear stress is smaller and takes place after a larger shear displacement magnitude. As a consequence shear stiffness, representing the first steep elastic part, would be smaller with increased sample size. Furthermore, the onset of shear dilation is delayed in larger samples because a larger 
displacement is required to reach peak shear stress. The peak shear strength depends on normal stress, with a higher peak stress for a higher normal stress. This was examined by Byerlee (1978) for normal stresses up to $100 \mathrm{MPa}$, who showed the relationship

$$
\frac{\sigma_{s c}^{\text {Peak }}}{\sigma_{n}}=0.85
$$

Barton and Choubey (1977) studied shear behavior at engineering stress levels and developed the following relationship:

$$
\frac{\sigma_{s c}^{m o b}}{\sigma_{n}}=\tan \left[J R C_{m o b} \log \left(J C S / \sigma_{n}\right)+\Phi_{r}\right]
$$

where $\sigma_{s c}{ }^{m o b}$ is the mobilized shear strength, $J R C_{\text {mob }}$ is the mobilized Joint Roughness Coefficient, JCS is the Joint Compressive Strength, and $\Phi_{r}$ is the residual friction angle. The term "mobilized" denotes current values of $\sigma_{s}$ and $J R C$ at any current shear displacement, which can be before, at or after peak-shear stress (Barton et al. 1985). The dilation curve ( $\Delta u_{n}$ versus $\left.\Delta u_{s}\right)$ can be calculated by the following expression:

$$
\begin{aligned}
& \Delta u_{n}=\Delta u_{s} \tan d_{m o b} \\
& d_{m o b}=\frac{1}{m} J R C_{m o b} \log \left(J C S / \sigma_{n}\right)
\end{aligned}
$$

where $d_{m o b}$ is the mobilized dilation angle and $m$ is a damage coefficient, given values of 1 or 2 for shearing under low or high normal stress, respectively (Olsson and Barton, 2001). Equations (33) - (35) and the size dependency of $J R C$ are the basic empirical equations of the Barton-Bandis joint model for fracture shear. 


\section{Hydromechanical coupling under normal closure}

Londe and Sabarly (1966) were perhaps the first to perform HM experiments on rock fractures during their investigation of the Malpasset Dam failure in the early 1960s (described later in the text). Experimental results typically show a decrease in fracture transmissivity with normal stress (Figure 4b), but with an apparent residual transmissivity, $T_{r}$, at high stress when the fracture appears to be mechanically compressed. The residual transmissitivy indicates that the fluid flow at high stress might be dominated by tube-like flow channels, which have a low aspect ratio and therefore cannot be closed easily; see Equation (16). In a few of the experiments (e.g., Kranz et al. 1979 and Iwano 1995) a value of Biot's $\alpha$ for fracture flow have been determined to be less than one and to vary with effective normal stress, with a higher $\alpha$-value at low stress.

Figure $4 a$ and $c$ show a size effect for normal closure. Witherspoon et al. (1979) suggested a size effect on the experimentally determined hydraulic properties of rock fractures. This suggestion was based on data from experiments carried out on an ultralarge core (0.95 $\mathrm{m}$ in diameter), an in situ block test $\left(1 \mathrm{~m}^{2}\right)$ reported by Pratt et al. (1977), and smaller laboratory samples (0.15 $\mathrm{m}$ in diameter) reported by Iwai (1976). Their results presented in Figure 5 showed that at the maximum stress level that could be attained $(10-20 \mathrm{MPa})$, the minimum values of fracture hydraulic conductivity were not the same for each rock specimen, but increased with specimen size. Barton and Bakhtar (1982) noted similar behavior in an in situ block experiment finding that the effective aperture could not be decreased to less than 30 microns at the maximum normal stress of $7 \mathrm{MPa}$. The effect of size on stress-permeability coupling of fractures was confirmed in 
theoretical studies by Neuzil and Tracy (1981) and Swan (1983), who predicted increasing permeability with sample size. Neuzil and Tracy (1981) attributed this size effect to a truncation of the aperture frequency distribution implying that fewer of the largest, least frequent flow channels would be included in a smaller sample. A conflicting observation regarding the size effect reported by Raven and Gale (1985) may result from unrepresentative sampling of the fracture surface asperities (Raven and Gale 1985).

Witherspoon et al. (1980) developed a modified cubic law, which they validated against laboratory experiments on artificial tension fractures in samples of granite and marble. They considered a general flow law

$$
\frac{Q}{\Delta h}=\frac{C}{f}(b)^{n}
$$

where $f$ is a friction factor that accounts for the roughness of the fracture surface, $b$ is an apparent physical aperture and $C$ is a constant depending on the flow domain geometry and the properties of the fluid (e.g. $C=\rho_{f} g w / 12 \mu_{f}$ for parallel flow, see Equation (13)). If $n=3$, this is a cubic law or modified cubic law, and in such case the apparent physical aperture is related to the hydraulic aperture as:

$$
b=f^{1 / 3} b_{h}
$$

Witherpoon et al. (1980) interpreted the apparent physical aperture as the residual aperture, $b_{r}$, plus an apparent mechanical opening according to

$$
b=b_{r}+\left(\delta_{\text {max }}-\delta\right)
$$

Using a slightly different approach, Elliot et al. (1985) parameterized the apparent physical aperture as 


$$
b=b_{0}-\delta
$$

where $b_{0}$ is the apparent physical aperture when $\delta$ is zero. A third approach used by Rutqvist (1995a) parameterized the apparent physical aperture as

$$
b=b_{i}+\left(\delta-\delta_{i}\right)
$$

where $b_{i}$ and $\delta_{i}$ are the apparent physical aperture and normal closure at initial effective stress. In the latter case the current hydraulic aperture can be calculated conveniently as

$$
b_{h}=b_{h i}+\frac{\Delta \delta}{f^{1 / 3}}=b_{h i}+f^{\prime} \Delta u_{n}
$$

where $f^{\prime}=1 / f^{1 / 3}, \Delta u_{n}$ is the normal deformation of the fracture from initial conditions, and $b_{h i}$ is the hydraulic aperture at initial effective stress.

Barton et al. (1985) criticized the use of an apparent physical aperture and proposed an empirical relationship:

$$
b_{h}=\frac{b_{E}^{2}}{J R C^{2.5}} \quad ; b_{E} \geq b_{h}
$$

where $b_{E}$ is the "real" physical aperture (also called "mechanical aperture" and usually having the symbol $E$ in the literature). Here the "real" physical aperture corresponds to the arithmetic mean of the separation of the two fracture surfaces, which can be measured directly with a feeler gauge. Equation (42) implies a nonlinear relationship between hydraulic and mechanical aperture, and hence a nonlinear relationship between $\Delta b_{h}$ and $\Delta u_{n}$. This is different from the approaches by Witherspoon et al. (1980), Elliot et al. (1985), and Rutqvist (1995a), which all have a linear relationship between $\Delta b_{h}$ and $\Delta u_{n}$ (or $\Delta \delta$ ). Intuitively, Barton's equation (42) is more correct. However, as demonstrated by 
Wei and Hudson (1988) and Zhao and Brown (1992), over an engineering stress range of $\sigma_{n}^{\prime}<10 \mathrm{MPa}$, the linear relationship seems to match experimental data as well as Barton's equation.

There has been conflicting laboratory evidence regarding the validity of the cubic law and the effects of contact area for fluid flow in rock fractures. As mentioned above, Iwai (1976) and Witherspoon et al. (1980) validated the cubic law against laboratory experiments on tension fractures in granite and marble. They found that the cube of the aperture is proportional to the flow if a correction is made for a residual aperture at high stress. In contrast, a number of studies during the 1980s and 1990s have shown a dramatic deviation from the cubic law (for example Engelder and Scholz (1981), Raven and Gale (1985), and Pyrak-Nolte et al. (1987)).

Boitnott (1991) carefully reviewed the conflicting evidence on the cubic law. He pointed out that much of the contradictory observations result from different data sets having been analyzed differently; the term "cubic law" does not have a consistent definition in the published literature. He used the general formulation of the flow law in Equation (36) and re-evaluated the conflicting data from 16 references of published data. He found that the predictions by the cubic law (i.e., $n=3$ and $f=1$ in Equation (36)) commonly holds for a wide variety of fractures. A significant number of cases, where the cubic law does not hold involve cases in which the hydraulic aperture appears to be less sensitive to closure than predicted by the cubic law. These cases can typically be fitted with a modified cubic law (i.e. Equation (36) with $\mathrm{n}=3$ and $f \neq 1.0$ ). In many cases, the 
modified cubic law breaks down as closure is increased, with these cases typically exhibiting a progressive decrease in the sensitivity of hydraulic aperture to further closure. Boinott (1991) concluded that this breakdown frequently occurs when the hydraulic aperture is less than about $50 \mu \mathrm{m}$. During the 1990s, these observations have been confirmed in several studies (e.g., Iwano (1995); Zhao and Brown (1992)). This shows that residual aperture is an important parameter for the coupled stress-flow behavior of rock fractures.

A complete relationship between fracture transmissivity and effective normal stress can be derived by combining a fracture flow law with an equation for fracture normal closure. For example, Rutqvist (1995a) combines Goodman's (1974) model in Equation (29) with Equations (26) and (41) to derive:

$$
T=C\left[b_{h i}+\frac{\sigma_{n i}^{\prime}}{\kappa_{n i}}\left(1-\frac{\sigma_{n i}^{\prime}}{\sigma_{n}^{\prime}}\right)\right]^{3}
$$

where $\kappa_{n i}$ was denoted as the initial "hydraulic normal stiffness," since it reflects how hydraulic aperture changes with stress. The hydraulic stiffness is related to mechanical stiffness by

$$
\kappa_{n i}=\frac{k_{n i}}{f^{\prime}}
$$

which shows that the hydraulic stiffness is usually equal to or less than the mechanical stiffness. Similarly, Alm (1999) derived a relationship between transmissivity and normal stress by combining Equations (26) and (31), leading to 


$$
T=C\left[b_{h 0}-\left(\frac{d k_{n}}{d \sigma_{n}^{\prime}}\right)^{-1} \ln \frac{\sigma_{n}^{\prime}}{\sigma_{n 0}^{\prime}}\right]^{3}
$$

In addition to the above models, there are a number of empirical and theoretical models to describe the relationship between normal stress across a fracture and its transmissivity. Models for macroscopic hydromechanical behavior of fractures have been developed from theories of microscopic behavior of rough fractures. These include the beds-of-nail model by Gangi (1978), the aperture frequency model by Neuzil and Tracy (1981), the quasi two-dimensional aperture-void model by Tsang and Witherspoon (1981), the Hertzian contact models by Walsh (1981) and Swan (1983), and stochastic models, e.g., Brown and Scholz (1986) and Brown (1987). Walsh's (1981) model, which is the most well known, can be written as:

$$
T=T_{0}\left[1-\left(\frac{\sqrt{2} h_{e}}{b_{0}} \ln \frac{\sigma_{n}^{\prime}}{\sigma_{n 0}^{\prime}}\right)\right]^{3} \tau_{w}\left(\Delta \sigma_{n}^{\prime}\right)
$$

where $T_{0}$ and $b_{0}$ are joint transmissivity, and aperture at some reference effective stress, $\sigma^{\prime}{ }_{n o}, h_{e}$ is the standard deviation of the asperity height distribution and $\tau_{w}$ is a tortuosity factor that depends on the normal stress. This equation is derived using Hertzian theory, assuming that the fracture consists of two uncorrelated rough surfaces, each with random topography of exponential distribution. Walsh (1981) studied experimental data of Kranz et al. (1979) and found that the tortuosity factor can be neglected for fluid flow, since aperture is raised to the third power and overwhelms the tortuosity term, which is only raised to the first power. If the tortuosity factor is neglected, and $b_{0}$ is assumed equal to $b_{h 0}$, then Equation (46) reduces to Equation (45), which is also equivalent to the empirical model developed in petroleum engineering by Jones (1975). The equivalence of 
Equations (45) and (46) in this case becomes obvious if $\left(b_{h 0}\right)^{3}$ is broken out of the square bracket in Equation (45) with the resulting $\left(b_{h 0}\right)^{3} \mathrm{C}=T_{0}$, and $\left(d k_{n} / d \sigma_{n}{ }^{\prime}\right)^{-1}=\sqrt{ } 2 h_{e}$ Equivalent logarithmic models have also been applied by Zhao and Brown (1992), and Evans et al. (1992) for hydromechanical normal closure behavior and by Bandis et al. (1983) for mechanical normal closure behavior of unmated rock joints. Therefore, it can be said that the theoretical logarithmic normal closure model by Walsh (1981) has been widely applied and that a logarithmic function seems to fit laboratory data, at least at engineering stress levels.

\section{Hydromechanical behavior during shear}

Laboratory measurements of hydromechanical behavior during shear have been rare because of a lack of specialized test equipment. The first comprehensive experimental study of permeability changes caused by shear was conducted by Makurat et al. (1990), who developed a coupled shear-flow test apparatus that could apply bi-axial stress. They concluded that whether the conductivity increases or decreases with shear depends on both the joint and rock properties, as well as the exact nature of the stress applied. Makurat et al. (1990) determined that decreases in hydraulic conductivity during shearing were a result of gouge production, which tended to block flow paths. The experiments were modeled using a Barton-Bandis joint model, which predicted an increasing aperture with shear dilation, according to

$$
b_{E}=b_{E i}+\Delta u_{n}
$$

where $\Delta u_{n}$ is the dilation of the fracture obtained from Equation (34) and $b_{E i}$ is the initial aperture before shear. This equation overpredicted increases in fracture permeability 
during shear because it does not correct for the formation of gouge material in the fractures.

Recent tests on granite reported by Esaki et al. (1999) and Olsson and Barton (2001) show that the joint transmissivity starts to increase after about $1 \mathrm{~mm}$ of shear and then increases rapidly by about 1 to 2 orders of magnitude up to $5 \mathrm{~mm}$ shear. These tests also indicated that the mechanical dilation of the fracture is greater than changes in hydraulic aperture. This has been attributed to asperity damage under increasing shear deformation with accompanying gouge production. As a result, Olsson and Barton (2001) found that Equation (42) relating hydraulic and real physical aperture breaks down after peak shear displacement. They presented a new tentative relationship expressed as

$$
b_{h}=\sqrt{b_{E} / J R C_{m o b}}
$$

which is valid after $75 \%$ of the shear displacement at peak stress. This relationship matched their laboratory data quite well.

\section{IN SITU HM CONDITIONS IN FRACTURED ROCK MASSES}

This section describes the in situ HM conditions found in fractured rock masses. They are related to the current distribution of in situ stress and permeability in the Earth's crust. In turn, the current in situ stress and permeability distributions are results of past and ongoing geological processes. However, in an engineering time frame they may be taken as initial quasi-static conditions. A correlation between in situ stress and permeability can indicate a coupling between the modern stress field and permeability. For example, a

decreasing permeability with depth or a correlation between anisotropy of principal stress 
and permeability fields may indicate that permeability is strongly dependent on stress. If on the other hand, permeability is correlated to maximum shear stress direction, it will indicate that fractures are prone to further shear slip following any human induced disturbance (for example during fluid injection or underground excavation).

\section{Conceptual model of crystalline fractured rock}

Fractured rock masses consist of discontinuities of various scales, from decimeter-size single joints to kilometer-size major fault zones. Therefore, mechanical and hydraulic properties can be and typically are strongly heterogeneous. As an example of a fractured rock mass, Figure 6 shows a conceptual model for the U.S. Geological Survey's fractured rock research site near Mirror Lake, New Hampshire. The investigations at Mirror Lake suggest that the bedrock contains a small number of highly transmissive fractures within a larger network of less transmissive fractures. The highly transmissive fractures appear to form local clusters. Each fracture cluster occupies a near-horizontal, tabular-shaped volume, which is several meters thick and 10 to $40 \mathrm{~m}$ across (National Research Council, 1996). The domination of a few connected fractures has been observed at many fractured crystalline rock sites (e.g., Stripa (Olsson et al. 1992); Forsmark (Carlsson and Olsson 1977), Laxemar (Rutqvist et al. 1997), and Äspö in Sweden (Rhén et al. 1997)). A clustered or compartmentalized system of highly conductive fracture networks has been reported from Kamaishi Mine, Japan (Doe 1999) and Sellafield, U.K. (Sutton et al. 1996). At the Forsmark site for low- and intermediate radioactive nuclear waste disposal in Sweden, a detailed observation of inflows was classified into five main types, as shown in Figure 7. Carlsson and Olsson (1977) found that most water inflow was related 
to features labeled 1 and 4 , which are associated with fractures opened by large-scale fracture surface undulation and fracture intersections.

Figure 8 provides a three-dimensional view of a highly permeable fracture. Hakami (1995) conducted extensive fracture aperture measurements of a minor fault intersecting a drift at Äspö Hard Rock Laboratory, Sweden. The fault belongs to a group of steep dipping fractures zones, typically 10 to $30 \mathrm{~m}$ long, of a type found to be the most conductive in the area. It is located at a depth of about $200 \mathrm{~m}$ and the minimum principal stress is about $5 \mathrm{MPa}$. The fault has been reactivated several times and contains fracture filling materials such as calcite, chlorite, epidote, and quarz. Measurements showed that the average aperture of the fracture (outside contact zones) was about $2 \mathrm{~mm}$, with the coefficient of variation $130 \%$. The contact area, defined as the area with aperture less than $0.1 \mathrm{~mm}$, covers about $40 \%$ of the total area of the fracture. The conceptual model in Figure 8 shows that the fault is undulating and "locked open" by a shear slip of about 4 $\mathrm{cm}$. This appears to belong to Flow Feature 1 in Figure 7.

\section{In situ HM behaviour from injection tests}

In situ HM properties can be inferred from a so-called "hydraulic jacking test" (also called a step-rate test) conducted in single boreholes. The technique was first applied by Londe and Sabarly (1966) and Louis et al. (1977) to study pressure-sensitive permeability under dam foundations. Rutqvist (1995b) and Rutqvist et al. (1997) used hydraulic jacking tests, combined with coupled numerical modeling, for determining the in situ HM properties of fractures in crystalline rocks. Hydraulic jacking tests, were conducted by a 
step-wise increase of the fluid pressure. At each step, the well pressure was kept constant for a few minutes until the flow was steady (Figure 9a). The numerical analysis of these injection tests shows that the flow rate at each pressure step is strongly dependent on the fracture aperture and normal stiffness of the fracture in the vicinity of the borehole, where the pressure changes the most (Figure 9b). Figure $9 c$ shows field test results of a hydraulic jacking test on a fracture at $267 \mathrm{~m}$ depth, in a crystalline fractured rock at Laxemar in Southeastern Sweden (Rutqvist et al. 1997). At the first cycle of step-wise increasing pressures, the flow rate increases as a nonlinear function of pressure. A temporal peak-pressure is obtained at a flow rate of 1.3 liters/minute before the pressure begins to decrease with an increasing flow rate. A shear-slip analysis of the particular fracture, which was inclined to the principal in situ stresses, indicated that these irreversible fracture responses could be caused by a shear slip, as the fluid pressure reduced the shear strength of the fracture. The subsequent step-pressure cycle took a different path because of the change in hydromechanical properties resulting from shearing and fracturing.

The overall results from the hydraulic jacking tests conducted at Laxemar showed that the pressure sensitivity of the fractures are strongly dependent on the initial hydraulic permeability. The permeability of the most conductive fractures is relatively insensitive to injection pressure, whereas the permeability of the least conductive fractures can be strongly dependent on the injection pressure. From the borehole-televiewer image, the most conductive fractures appear to be open fractures that are incompletely cemented, 
indicating flow channels in a fracture that are "locked open" by shear dislocation or mineral filling (Rutqvist et al. 1997).

There are a few examples where hydraulic jacking tests have been conducted with simultaneous mechanical measurements of fracture deformations (Gale 1975; Jung 1989; Martin et al. 1990; Myer 1991). Jung (1989) measured fracture hydraulic and mechanical widths during hydraulic injection into a large hydraulic fracture (over $100 \mathrm{~m}$ in diameter) located at about $250 \mathrm{~m}$ depth in granite. The fracture has a dip of about $45^{\circ}$ relative to the horizontal plane and the normal stress across the fracture was estimated to 4.4 MPa. Figure 10 presents hydraulic and physical apertures determined at one intersecting boreholes a function of fluid pressure in the fracture. The figure shows that at the initial hydrostatic fluid pressure (corresponding to an effective normal stress of 1.9 MPa), the hydraulic conducting aperture is about $0.5 \mathrm{~mm}$ while the apparent physical aperture is about $1 \mathrm{~mm}$. At fracture extension pressure (corresponding to $0.3 \mathrm{MPa}$ above the effective normal stress), both hydraulic and physical aperture is estimated to be around $2.0 \mathrm{~mm}$ (Figure 10). The very large residual aperture indicated in Figure 10, seems to agree with a general size effect discussed Previously and may also have been caused by a fracture shear slip during the hydraulic injection.

\section{Possible correlation between permeability and depth (stress)}

Figure 11 presents a profile of short interval (3-meter packer separation) well tests performed at a typical granitic rock for the site investigation of the Swedish radioactive waste disposal program, located at Gideå, Sweden. The figure may show a general 
decrease in permeability with depth, however, the depth dependency is obscured by a very large permeability spatial variation (up to 6 orders of magnitude). A general observation from Figure 11, and from many fractured crystalline rock sites, is that the most pronounced depth dependency can be found in the upper 100 to $300 \mathrm{~m}$ of the bedrock. It has been suggested that this depends on near-surface fracturing owing to stress relief caused by erosion and isostatic rebound after glaciation, enhanced by weathering and solution of fracture minerals (Wladis et al. 1997). However, the more pronounced depth dependency in shallow areas can also be explained by the nonlinear normal stress-aperture relationship of single extension joints (Figure 4b). Such a decrease in hydraulic aperture with depth has been observed by Snow (1968), who used detailed pumping tests and fracture statistics to determine fracture apertures at numerous dam foundations. His results showed that the hydraulic aperture decreases from about $200 \mu \mathrm{m}$ at the ground surface to about $50 \mu \mathrm{m}$ at $60 \mathrm{~m}$ depth.

The permeability in Figure 11 varies from $5 \times 10^{-19}$ to $4 \times 10^{-13} \mathrm{~m}^{2}$. The minimum values on the left-hand side of Figure 11 represent the permeability of the rock matrix, indicating that no fracture intersected the test interval, or if fractures are intersecting, they are either completely cemented with minerals or isolated from a conducting fracture network. The maximum permeability values on the right-had side of Figure 11, represent the transmissivity of at least one intersecting fracture, which is highly conductive and connected to a larger network of conducting fractures. As discussed previously, these highly conductive fractures are likely "locked-open" either by bridges of hard mineral filling or by large shear dislocation. The fluid flow in these "locked-open" fractures take 
place in channels between bridges, and are likely to be relatively insensitive to stress. The phenomenon of "locked open" fractures is well known in hydrocarbon reservoirs, especially in carbonate rocks, where localized dissolution of the wall rock can create highly conductive pathways of several millimeters even at high in situ stress (Dyke 1995). In crystalline rocks, the "locked open" fracture category could occur in mineral-

filled or shear-dislocated fractures throughout the rock mass. However, large channels are more likely to occur in fault zones where large movements allow substantial shear dislocation and extension of oblique fractures (National Research Council 1996). It has also been observed that highly permeable fracture zones are relatively insensitive to stress compared to competent fractured host rock media (Carlsson and Olsson 1986; Rutqvist et al. 1997).

\section{Correlation between anisotropy of in situ stress and permeability}

An in situ stress-dependent permeability has been inferred from observed in situ stress and permeability anisotropies. One example is a study at an underground hydropower plant in Juctan, Sweden (Carlsson and Olsson 1979). The study, which was conducted at about $250 \mathrm{~m}$ depth in a mountain of granitic rock, showed that the anisotropic hydraulic conductivity of the fractured rock was correlated with the anisotropic in situ stress field. An anisotropy factor of $3\left(k_{H}=1.9 \times 10^{-9} \mathrm{~m} / \mathrm{s}\right.$ and $\left.k_{h}=0.6 \times 10^{-9} \mathrm{~m} / \mathrm{s}\right)$ was obtained for the hydraulic conductivity in a stress field that showed a local anisotropy factor of 4.3 $\left(\sigma_{H}=15 \mathrm{MPa}\right.$ and $\left.\sigma_{h}=3.5 \mathrm{MPa}\right)$. One reason to believe that this anisotropy of permeability was in fact stress induced is that the measured in situ stress field (which probably was affected by the nearby excavation) was extremely anisotropic. Most 
importantly, the minimum horizontal stress was very low, which implies that some fractures are in a low normal stress (low normal stiffness) regime, in which permeability can be highly stress dependent.

Another example is provided by a study of a fractured oil reservoir in the Ekofisk Field in the North Sea (Teufel and Farrel 1995). The top of the reservoir is at a depth of $2.9 \mathrm{~km}$, and reservoir thickness is up to 305 meters. The reservoir consists of two fractured chalk intervals separated by a relatively impermeable layer of unfractured chalk; see also Lewis et al. (2003) in this Special Issue. Fractures with two distinct orientations were logged from boreholes, with one set far more abundant than the other. Hydraulic testing showed that the direction of maximum principal permeability was aligned with the less abundant fracture set, and that its direction was parallel to the maximum compressive stress. An anistropy factor of $4.4\left(k_{H}=1.59 \times 10^{-13}\right.$ and $\left.k_{h}=3.6 \times 10^{-14} \mathrm{~m}^{2}\right)$ in permeability was obtained for a stress field with an anisotropy factor of about 1.8 (calculated using Equation (1) with $\sigma_{H}=40 \mathrm{MPa}, \sigma_{h}=33 \mathrm{MPa}$, and $p=24 \mathrm{MPa}$ ) for effective stresses. In this case the minimum compressible stress is about $9 \mathrm{MPa}$, which implies that fractures should be relatively compressed close to a residual aperture. Thus, it might be questioned whether the permeability anisotropy observed by Teufel and Farrel (1995) really results from a present-day stress field. Furthermore, it appears that the permeability has remained constant at Ekofisk despite a $22 \mathrm{MPa}$ reduction in pore pressure over 18 years of production (Dyke 1995), which indicates that the permeability at reservoir depth is not particularly stress dependent. 
Stowel et al. (2001) questioned whether modern-day stress controls the orientation of the most conductive fractures in deep reservoirs. They reviewed stress, fracture, and production data from low- to moderate-matrix-permeability reservoirs at three sites in the Western United States. Their comparison of stress directions and orientation of flowcontrolling fractures showed that open fractures in the subsurface are not necessarily parallel to the maximum principal compressive stress and that fractures parallel to the maximum principal stress are numerous. They referred to partially cemented "lockedopen" fractures as the most important flow features at depth. The findings by Stowel et al (2001) are not surprising, especially since their data came from depths of 2,400 to 6,400

m. As noted in the previous section, the depth dependency of permeability appeared only for the upper $100-300$ meters, whereas at greater depth fractures are closed to a residual permeability value. This residual permeability value is very high for "locked open" fractures, but their stiffness is high because of hard-mineral bridges. Hence, at these depths, bulk permeability cannot be expected to be especially stress sensitive.

\section{Possible correlation between maximum shear stress and permeability}

In the last decade, investigations of the active lithospheric-plate boundary in California have shown that fractures favorably oriented for shear slip, so-called critically stressed fractures, tend to be active groundwater flow paths (Barton et al. 1995, Barton et al. 1998 and Ferrill et al. 1999). The rationale for bulk permeability being dominated by critically stressed fractures is that most fractures in the bedrock are cemented because of water/rock chemical reactions. If shear slip occurs on a critically stressed fracture, it can raise the permeability of the fracture through several mechanisms, including brecciation, 
surface roughness, and breakdown of seals (Barton et al. 1995). Lately, similar correlations have been found at the KTB Scientific Drill Hole in Germany down to $7 \mathrm{~km}$ (Ito and Zoback 2000), and at Äspö, Sweden, in Precambrian rocks of the Baltic Shield deep in the Eurasian plate (Talbot and Sirat 2001). On the other hand, experience from injection experiments at the hot-dry rock geothermal sites in Soultz, France, and Rosemanowes, U.K., suggests that a pore-pressure increase of 5-6 MPa over ambient is needed to stimulate significant microseismicity (Evans et al. 1999). This indicates that under undisturbed stress and pressure conditions, the fractures would not be at the verge of shear failure.

\section{OBSERVATIONS OF HUMAN INDUCED HM INTERACTIONS IN}

\section{THE EARTH CRUST}

This section introduces field observations of HM interactions in fractured rock masses triggered by human activities. All these observations (e.g. landsubsidence due to fluid extraction, injection-induced shear failure and seismicity, and excavation-induced squeezing) are evidence of direct HM couplings accompanied by indirect couplings. Thus, the coupled responses are triggered by changes in stress or fluid pressure and are accompanied by changes in material properties (see Figure 2).

\section{Land subsidence due to fluid extraction}

Evidence of direct fluid-to-solid coupling is perhaps most obvious in land subsidence caused by underground fluid removal. In this case, land subsidence is caused by a reduction in fluid pressure that increases the effective stress, which in turn results in a 
contraction of the geological media. However, the land subsidence process can be quite complex, and the amount of subsidence during fluid removal depends on a number of factors (such as size and depth of the exploited formation, magnitude of the pressure decline, compressibility, and permeability of the rocks). One of the most dramatic examples of land subsidence has occurred in Mexico City caused by groundwater withdrawal and associated aquifer compaction. The maximum rate of subsidence approaches $60 \mathrm{~cm}$ per year, with total subsidence during the $20^{\text {th }}$ century as great as 10 meters. (Galloway et al. 1999). In the United States, more than $80 \%$ of the identified subsidence results from exploitation of underground water resources, with the agriculture-intensive San Joaquin Valley, California, being the largest (Galloway et al. 1999). As of the last geodetic survey (1970), subsidence in excess of one foot (0.3 m) affected more than 5,200 acres $\left(21 \mathrm{~km}^{2}\right)$, with the maximum subsidence more than $9 \mathrm{~m}$. Although rare, dramatic land subsidence has also been observed during oil- and gas exploration. One well-known example is the Wilmington Oil Field in California, which experienced a subsidence of almost $9 \mathrm{~m}$ from 1926 to 1967 (Figure 12). The amount of subsidence at the Wilmington Oil Field was substantial because of a significant reduction of the reservoir pressure over a large vertical interval. More recently, oil and gas extractions at the Ekofisk Field in the North Sea have caused a subsidence of the sea bottom of more than $10 \mathrm{~m}$ since the 1970s and have necessitated to repeated large volume seawater injections to jack-up the platforms. Lewis et al. (2003) in this issue presents the subsidence of Ekofisk Field in more detail. 


\section{Seismicity and failure associated with deep well fluid extraction/injection}

Seismicity caused by fluid extraction has been observed during oil production at numerous oil-fields since the 1920s (Segall 1989) and more recently during geothermal energy extraction (Segall et al. 1998). Extraction induced earthquakes are triggered by poroelastic stresses associated with reservoir contraction. Declining pore pressure causes reservoir rocks to contract slightly with associated reduction of the horizontal stress. Figure 13 presents field data showing such fluid pressure-stress coupling in the Ekofisk Field and for the McAllen range, Texas field. Because the reservoir is elastically coupled to the surrounding rocks, the contraction of the reservoir and the reduction of stress in the reservoir is balanced by increased stress in the neighboring crust. This stress results in subsidence, horizontal contraction above the reservoir, and in some instances, triggered seismicity (Segall 1989). Examples of faulting associated with fluid withdrawal are illustrated schematically in Figure 14. One of the most obvious examples of seismicity caused by fluid extraction is the Wilmington Oil Field where spectacular surface deformation was accompanied by seismicity. Six shallow earthquakes (M 2.4 to 3.3) occurred within the oil field between 1947 to 1955, when the rate of subsidence was greatest (Segall 1989). The earthquakes were generated by slip on bedding planes at a depth of 470 to $520 \mathrm{~m}$, which sheared off tens of oil wells over several square kilometers (Segall 1989). Although the earthquakes at Wilmington Oil Field caused equipment damage, their magnitude was moderate. However, oil production has also been attributed to more substantial seismic activity around the world (Cypser and Davis 1998). 
Seismicity caused by fluid injection has been observed at sites used for deep injection of hazardous waste, during reservoir stimulation and during forced circulation in geothermal fields. A classic example of injection-induced seismicity occurred at a deep (3,638 m) well for liquid-waste injection at the Rocky Mountain Arsenal near Denver, Colorado (Hsieh and Bredehoeft 1981). During the first months of operation in 1962, over 15,000 $\mathrm{m}^{3}$ were injected, with a wellhead pressure ranging from 0 to 7.2 MPa. In March 1962, Denver experienced its first earthquake in 80 years. The injection continued, off and on, until Evans (1966) publicly suggested a correlation between fluid injection and seismic activity. The correlation was observed through seismic monitoring, which showed that seismic activity generally increased during injection periods and declined during periods of no injection (Hsieh and Bredehoeft 1981). However, the three largest earthquakes occurred at the site more than one year after the injection was discontinued, in 1967, with magnitudes of M 4.8 and 5.3 (Cypser and Davis 1998). The continuous seismicity years after injection stopped was explained by postinjection propagation of built-up injection pressure throughout the reservoir, which could reach and release strain from tectonically stressed faults (Healy et al. 1968).

Since the Denver Basin earthquakes, many injection-induced quakes have been documented (Nicholson and Wesson 1992). However, of the thousands of injection wells in the world, only a few have induced notable earthquakes. Such notable examples are an experimental well in the Chevron Oil Field near Rangely, Colorado and sites at El Dorado, Arkansas. 
Intentional hydraulic stimulation and microseismic monitoring have also been applied at fractured geothermal (hot-dry rock) sites since the first site was developed in Fenton Hill, New Mexico, in the late 1970s. Results from the monitoring of hydraulic fracturing experiments have been reported by Aki et al. (1982) from $3.5 \mathrm{~km}$ deep large-volume injection at Fenton Hill (U.S.), by Pine and Bachelor (1984) from $2.5 \mathrm{~km}$ deep, largevolume injection done in Rosemanoes (U.K.), by Jupe et al. (1992) from $0.5 \mathrm{~km}$ deep injection at Fjällbacka (Sweden), and by Gaucher et at. (1998) from Soultz-souz-Forêts (France), and several other sites worldwide.

An illustrative example is presented in Figures 15 and 16 from the Hijiori hot-dry rock site in Japan. Figure 15 shows flow, pressure, and seismic activities associated with two different experiments: a one-day high-pressure hydraulic-fracturing experiment, performed in 1988, and a one month low pressure circulation experiment performed in 1989. In both, the seismic rate (Figure 15) appears to be strongly dependent on well-head pressure. However, the spatial distribution of the microseismic events in the two experiments is quite different (Figure 16). Microseismic events induced by the 1988 hydraulic fracturing migrated towards the east and distributed along a vertical plane with a strike nearly parallel to the direction of the maximum principal stress. This indicated that pre-existing fractures were reopening along a zone that developed in the direction of maximum principal stress, although micro-seismic events were caused by shear slip. Seismicity accompanying the 1989 circulation test with a low injection pressure was diffuse, forming a seismic "cloud." It was concluded that the seismic cloud 
accompanying the circulation test resulted from the permeation of water into joints, which slipped when the effective stress was reduced by the increased fluid pressure.

\section{Seismicity and failure associated with reservoir impoundment}

Substantial seismicity and large-scale failure of rock masses have on several occasions been linked to surface reservoir impoundment (Gupta 1992). The process is analogous to fluid injection, in that increased fluid pressure will reduce the effective stress and shear strength along existing discontinuities, leading to shear failure or fracturing. In addition, the added loads from the reservoir water increases stress in surrounding rock formations and load dam abutments through direct elastic and poroelastic effects. Large-scale failure of rock masses during reservoir impoundment can be devastating. Examples are landslides (e.g., Vaiont, Italy, 1963) and dam failures (e.g., Malpasset, France, 1959). A landslide or dam failure may be preceded by induced seismic activity that can be a warning signal for a larger failure. However, large failures may also take place abruptly without forewarning and can be catastrophic.

In rare instances reservoir impoundment has been linked to some of the largest and most damaging earthquakes induced by humans (Gupta 1992). The first real evidence relating increased seismicity to water storage came in 1936, when an increase of seismicity occurred after the creation of Lake Mead, held back by the Hoover Dam on the ArizonaNevada border (Carder 1945). Since then, numerous such cases have been reported, and seem to fall into two categories of mechanical earth-crust response (Simpson et al. 1988). In many cases, there is a rapid response, in which seismicity increases almost 
immediately after reservoir impoundment. In other cases, there is a delayed response, in which the main seismic activity does not occur until some years after the reservoir has been filled and the water level is maintained at a stable height. Cases of rapid response tend to produce swarms of small earthquakes, located at shallow depth, in the immediate vicinity of the reservoir. In the delayed response case, the earthquakes tend to be larger, at a greater depth, and are often located at some distance from the deep part of the reservoir. An example of delayed response, and one of the most devastating earthquakes induced by reservoir impoundment, occurred at a dam that was being built in Konya, India containing Shivajisagar Lake. Prior to the dam being built, the area recorded very low seismic activity. After being filled in 1962, seismograph records showed frequent earthquakes directly beneath the lake. However, large earthquakes did not occur until late 1967, when magnitude 5.5 and 6.2 events occurred. The largest earthquake, which caused considerable damage and claimed the lives of 200 people, occurred at a depth of $5 \mathrm{~km}$, some $10 \mathrm{~km}$ downstream from the dam. It occurred just after the water level had reached its maximum value (Scholz 1990).

The best-known example of dam failure is the collapse of the Malpasset Dam, in France in 1959 that killed at least 500 people. The Malpasset Dam failure took place in a sudden movement, like an explosion, with nothing abnormal detected on the dam a few hours before the failure (Londe 1987). Six years of research showed that under the load of the dam, permeability was reduced by a factor of approximately 0.01 . As a result an "underground hydraulic barrier" was created, in which the concentrated action of seepage forces contributed to failure by sliding of the dam foundation along a fault located under 
the structure (Figure 17). At the time of the design and even at the time of the failure, very little was known about the mechanism of water seepage through fractured media. Foundation design procedure did not include any study of uplift in the rock, except at the contact between concrete and foundation (Londe 1987). Furthermore, the variation in rock permeability as a function of applied stress was an unknown phenomenon before the elaborate and systematic analysis undertaken as a result of the accident (Habib 1987). It was during the investigation of the Malpasset Dam failure that the concept of fractured rock hydraulics and stress-permeability coupling in single fractures was initiated.

\section{Excavation induced squeezing}

Solid-to-fluid interaction can be directly observed in a porous medium as a pressure pulse, if the porous medium (soil and rock) is squeezed rapidly by a mechanical force. For example, a rapid contraction of porous media leads to a local pressure increase. Fluid pressure can then diminish as fluid flows out of the pressurized area. An observable pressure pulse will only occur if the loading is so rapid and the permeability is so small that the fluid has no time to escape. One example of this phenomenon is the response in water-well levels caused by ground-compression of passing trains, studied by Jacob (1940). Figure 18 presents another recent example, which was observed in fractured crystalline rocks at the Grimsel Test Site for geological disposal of radioactive waste, Switzerland. The figure shows fluid-pressure response in a drift wall during boring of the drift (2.28 $\mathrm{m}$ in diameter). The fluid pressure was monitored in a packed-off borehole section, about $10 \mathrm{~m}$ long, oriented parallel to the future drift, at a radial distance of about $3 \mathrm{~m}$ from the drift wall (Figure 18). The figure shows two distinct pressure peaks, which 
occurred during two consecutive eight hour working shifts as the face of the tunnelboring machine (TBM) passed parallel to the monitoring section. The pressure peaks can be explained by an increased mean stress at or near the location of the packed-off monitoring section. Such increase in mean stress is possible if the virgin stress around the drift is anisotropic. After the end of each shift, when the excavation was stopped, the fluid pressure declined because fluid slowly diffused away from the pressurized zone. Similar transient phenomena have also been observed during excavations in Opalinus clay at Mont Terri Rock Laboratory, Switzerland (Thury and Bossart 1999), and in fractured granite at the Underground Research Laboratory (URL), Canada (Read and Chandler 1999).

\section{Excavation induced permeability changes}

Stress-induced changes in permeability around excavations have been studied since the early 1980s. These studies have been related to research on the excavation disturbed zone (EDZ) around tunnels, which is an issue of concern for the safety assessment of nuclear waste repositories. The effect of the EDZ on hydraulic behavior around tunnels was first realized in an experiment at the Stripa Mine, Sweden, as a part of the Stripa Project (see Witherspoon (2000) for recent recap). The experiment was a large-scale permeability test, in which the radial head distribution showed a peculiar drop within $5 \mathrm{~m}$ of the drift wall. This head decrease could be explained by a "skin," or zone of reduced permeability around the drift. This skin was thought at the time to reflect closure of fractures caused by tangential stress concentration around the opening. It was later found that as the water pressure fell below $17 \mathrm{~m}$ of water head outgassing occurred, and thus the increased 
gradient, just outside the wall, could also be attributed to effects of two-phase conditions in the fractures.

After this first observation at the Stripa Project, EDZs have been investigated at many sites in various rock types. A large fraction of these studies have involved measurements of mechanical responses during mine-by experiments or seismic velocity evaluation of a damaged zone due to blasting. In other studies, the mechanical and hydraulic responses have not been measured directly, but a skin zone around the excavation has been inferred in order to explain hydraulic behavior (e.g. Buffer Mass Test and Site Characterization and Validation Test in Stripa by Börgesson et al. (1992) and Olsson et al. (1992)). These damaged-zone observations and the suspicion of a permeability skin motivated deliberately designed in situ tests to quantify permeability changes around underground openings. Such in situ tests have been conducted since the 1990s at the Äspö hard rock laboratory, Sweden (Emsley et al. 1997), Kamaishi and Tono Mines in Japan (Sugihara et al. 1999) and at the Underground Research Laboratory in Manitoba, Canada (Bäckblom and Martin 1999).

Findings of the above mentioned experiments lead to a few general observations regarding EDZs and excavation-induced-permeability changes (Bäckblom and Martin 1999). First, the EDZ is the rock zone where rock properties and conditions are changed because of processes induced by excavation. The EDZ includes a damaged zone of induced rock failure and fracturing stemming from excavation processes, an unsaturated zone, a zone with altered stress distribution around the drift, and a zone of reduced fluid 
pressure (Figure 19). In the case of excavation-induced damage, high transmissivity will probably develop close to the opening. For mechanical excavation (using no blasting) in a moderate stress environment, the damage zone may be limited to a few centimeters thickness where a limited change in porosity and permeability may take place. When drill-and-blast is used for excavation, the damage zone is more extensive and therefore increased permeability is likely, especially in the floor, where the permeability can increase by two to three orders of magnitude. In a high stressed, relatively intact rock conditions like at the URL in Canada, induced fracturing and rock loosening at the wall of excavations can cause dramatic (up to six orders of magnitude) permeability changes. For the rock mass outside the damage zone, the experiments at Äspö, Tono, Kamaishi Mine, and URL indicate very little excavation-induced changes in permeability. For example, at the ZEDEX experiment in Äspö, the permeability sometimes increased and sometimes decreased before and after excavation, and it was not possible to come to a firm conclusion about changes in properties (Bäckblom and Martin 1999).

A recent study in unsaturated fractured tuff at Yucca Mountain, Nevada, provides a comprehensive data set pertaining to excavation-induced permeability changes (Wang et al. 2001). The data include measurements of air permeability at four niches excavated by a mechanical (alpine mining) method. Permeability was measured before and after excavation in $30 \mathrm{~cm}$ packed-off sections along $10 \mathrm{~m}$ long boreholes located about 0.65 to $1 \mathrm{~m}$ above the drifts, supposedly outside the damaged zone (Figure 20a). The results showed that measured air permeability, which generally ranged from $1 \times 10^{-15}$ to $1 \times 10^{-}$ ${ }^{10} \mathrm{~m}^{2}$ (Figure 20b), which is at least 3 orders of magnitude higher than the matrix 
permeability measured from core sample tests. This shows that at least one hydraulic conductive fracture intersects each $0.3 \mathrm{~m}$ borehole section and that the fractures, which are practically dry at all times, form a well-connected network for air flow. The results in Figure $20 b$ show that the pre/post-permeability ratio ranges between a factor of 1 to 400 , with a change in arithmetic mean of about 60 . There is also a tendency for stronger permeability increases in those sections where the initial permeability is small. Preliminary analyses by Wang and Elsworth (1999) and Rutqvist and Tsang (2002) show that the permeability increase can be explained by opening of horizontal fractures unloaded by the drift. A relatively large change in permeability around the drifts at Yucca Mountain, in relation to the other sparsely fractured sites mentioned above, is possibly caused by a well-connected fracture network that can create consistent changes in bulk permeability.

\section{Pressure sensitive permeability during injection/withdrawal into fractured reservoirs}

Pressure-dependent permeability for injection pressures below lithostatic stress have been confirmed at hot-dry rock-sites (e.g, Fenton Hill US (Brown 1993), Rosemanowes UK (Richards et al. (1994)), Fjällbacka Sweden (Eliasson et al. 1990), Higashihachimantai, Japan (Hayashi and Abe 1989), Falkenberg, Germany (Jung 1989) and Soultz, France (Baria et al. 1999a). At a geothermal site, transmissitivity is one of the main factors gove rning production of heat, and consequently, the economics of the operation. The hydraulic properties of a hot-dry rock reservoir are usually evaluated using hydraulic jacking tests to assess pressure dependency of transmissivity. Unlike the hydraulic 
jacking test mentioned earlier, where one fracture is isolated and pressurized, injectivity tests in geothermal reservoirs are large-scale and involve jacking of a large volume of fractured rock. Secondly, a circulation test may be conducted between two deep wells to determine the "flow impedance," which is defined as the pressure difference between the injection and production well divided by the injection rate (Hayashi and Abe 1989). It has been shown that flow impedance can improve if a back-pressure is applied to the production well, as a result of pressure-dependent permeability (Eliasson et al.1990, Brown 1993).

Examples of how permeability can depend on reservoir pressure have been recorded in fractured sedimentary "tight sand" gas reservoirs. One prime example is the U.S. Department of Energy-supported Multiwell Experiment (MWX) at the Piceance Basin in Colorado. The site was developed to perform detailed experiments on all aspects of lowpermeability natural gas reservoir evaluation, stimulation, and production (National Research Council 1996). One dominating set of extensional fractures, some of which are incompletely cemented, are the primary production sources for these tight sands. Interference tests showed that permeability anisotropy was on the order of 1:100, owing to the unidirectional nature of the fracture system. Production tests showed that the natural fracture system is highly stress sensitive. By decreasing the reservoir pressure below a critical value (typically about $6.9 \mathrm{MPa}$ ), the well production could be almost totally stopped because the decrease in pressure created higher effective stresses that physically closed the fractures (National Research Council 1996). 
Pressure-independent permeability in fractured oil and gas reservoirs has been reported in rock having highly conductive "locked open" fractures. Dyke (1995) listed six naturally fractured hydrocarbon reservoirs, which have been producing for a considerable time, under large drawdown pressure, without any noticeable reduction in permeability. Dyke (1995) argued that highly permeable macrofractures appear to control permeability in many reservoirs. These are irregularly distributed, and because of their scarcity do not belong to any common statistical grouping. Dyke (1995) concluded that the most reliable way characterizing the response of a fracture subset is through back-analysis of in-situ reservoir behavior.

\section{MODELING OF HM COUPLING IN FRACTURED ROCKS}

The advancement of computer technology in recent decades has made it possible to analyze problems using fully coupled HM analysis (including nonlinear, inelastic processes and heterogeneous geological media). This is of great benefit for practical applications. Jing and Hudson (2002) provide a comprehensive review of numerical methods in rock mechanics, including finite element (FEM), boundary element (BEM) and distinct element (DEM) methods. For more details on FEM modeling related to consolidation of porous media, a textbook by Lewis and Schrefler (1998) is recommended. For analytical solutions of HM coupling, refer to Neuzil (2003) regarding geological processes, and Wang (2000), who presents useful analytical solutions for a large number of practical problems. This section briefly introduces a coupled HM numerical model that uses a FEM technique and discusses some conceptual approaches for using equivalent continuum medium to represent HM behavior in fractured rocks. 


\section{Governing hydromechanical equations for porous deformable medium}

The macroscopic hydromechanical behavior of intact porous rock can be described as governed by a static force equilibrium:

$$
\nabla \cdot \boldsymbol{\sigma}+\rho_{m} g=0
$$

and by Biot's constitutive equation for mechanical behavior, written in incremental form as

$$
\boldsymbol{\sigma}=\mathbf{D}: \boldsymbol{\varepsilon}-\alpha \mathbf{I} p
$$

where $\mathbf{I}$ is the identity tensor and $\alpha$ is the Biot-Willis coefficient. This equation is more general then Equation (8) in that the medium does not necessarily have to be isotropic elastic. To accommodate for non-linear or plastic mechanical behavior, Equation (50) can be written in incremental form as

$$
d \boldsymbol{\sigma}=\mathbf{D}: d \boldsymbol{\varepsilon}-\alpha \mathbf{I} d p
$$

where $\mathbf{D}$ is the tensor of incremental elastic constants. For a linear elastic isotropic medium, the $\mathbf{D}$ can be Expanded in Cartesian coordinates as:

$$
\mathbf{D}=\left[\begin{array}{cccccc}
\frac{2 G(1-v)}{1-2 v} & \frac{2 G v}{1-2 v} & \frac{2 G v}{1-2 v} & 0 & 0 & 0 \\
& \frac{2 G(1-v)}{1-2 v} & \frac{2 G v}{1-2 v} & 0 & 0 & 0 \\
& & \frac{2 G(1-v)}{1-2 v} & 0 & 0 & 0 \\
& & & 2 G & 0 & 0 \\
& & & & 2 G & 0 \\
& & & & & 2 G \text { metric } \\
& & & & &
\end{array}\right]
$$

Equation (49) and (51) is combined to obtain the stress-compatibility equation, which for a quasi-static coupled system can be written in a general incremental form as: 


$$
\nabla \cdot\left(\mathbf{D}: \frac{\partial \boldsymbol{\varepsilon}}{\partial t}-\mathbf{I} \alpha \frac{\partial p}{\partial t}\right)=\frac{\partial \mathbf{F}}{\partial t}
$$

This equation basically assures that the internal net force (left hand side) in all directions are in balance with external forces and gravity effects (right hand side) working in the same direction.

The governing fluid flow equation can be written as:

$$
\nabla \cdot\left(\frac{\mathbf{k}}{\mu_{f}} \nabla\left(p+\rho_{f} g z\right)\right)-\alpha \frac{\partial \varepsilon_{v}}{\partial t}-\frac{1}{M} \frac{\partial p}{\partial t}=Q
$$

which is a combination of Equation (9) and Darcy's law.

\section{Finite element formulation}

The following presents a numerical formulation for coupled hydromechanical analysis as it was developed in the HM version of the ROCMAS code (Noorishad et al. 1982, 1992).

The finite element formulation for the matrix rock is expressed in terms of strain vector rather than the strain tensor leading to the following two equations:

$$
\begin{aligned}
& \nabla \cdot\left(\mathbf{D} \frac{\partial\{\varepsilon\}}{\partial t}\right)-\nabla \cdot\left(\alpha \mathbf{I} \frac{\partial p}{\partial t}\right)=\frac{\partial \mathbf{F}}{\partial t} \\
& \nabla \cdot(\mathbf{k} \nabla p)-\alpha \frac{\partial \varepsilon_{V}}{\partial t}-\frac{1}{M} \frac{\partial p}{\partial t}=Q
\end{aligned}
$$


where $\{\varepsilon\}$ is a column vector containing normal and shear strains (e.g. in Cartesian coordinates $\{\varepsilon\}=\left\{\varepsilon_{\mathrm{xx}}, \varepsilon_{\mathrm{yy}}, \varepsilon_{\mathrm{zz}}, \varepsilon_{\mathrm{xy}}, \varepsilon_{\mathrm{yz}}, \varepsilon_{\mathrm{zx}}\right\}^{t r}$ where $t_{r}$ denotes transpose). In Equation (56) the effect of gravity flow is included in the source term $Q$.

Using standard finite element procedures Equations (55) and (56) are discretized in terms of unknown nodal displacements, pressure, forces and flow through appropriate shape (interpolation) functions as:

$$
\left[\begin{array}{cc}
0 & 0 \\
0 & \hat{\mathbf{k}}_{\mathbf{P P}}
\end{array}\right]\left\{\begin{array}{l}
\hat{\mathbf{u}} \\
\hat{\mathbf{p}}
\end{array}\right\}+\left[\begin{array}{ll}
\hat{\mathbf{C}}_{\mathbf{u u}} & \hat{\mathbf{C}}_{\mathbf{u P}} \\
\hat{\mathbf{C}}_{\mathbf{P u}} & \hat{\mathbf{C}}_{\mathbf{P P}}
\end{array}\right] \frac{\partial}{\partial t}\left\{\begin{array}{l}
\hat{\mathbf{u}} \\
\hat{\mathbf{p}}
\end{array}\right\}=\left\{\begin{array}{c}
\partial \hat{\mathbf{F}} / \partial t \\
\hat{\mathbf{Q}}
\end{array}\right\}
$$

where the coefficient matrices $\hat{\mathbf{C}}_{\mathbf{u u}}, \hat{\mathbf{k}}_{\mathbf{P P}}$ etc are coefficient matrices that contains material parameters such as permeability, Biot's constants, appropriate shape and transformation functions and element volume. For the exact expressions of these coefficient matrices refer to Noorishad (1982, 1992).

Equation (56) is then integrated between time $\mathrm{t} 1$ and $\mathrm{t} 2=\mathrm{t} 1+\Delta \mathrm{t}$ to obtain:

$$
\left[\begin{array}{cc}
0 & 0 \\
0 & \Delta t \hat{\overline{\mathbf{k}}}_{\mathbf{P P}}
\end{array}\right]\left\{\begin{array}{l}
\hat{\mathbf{u}} \\
\hat{\mathbf{p}}
\end{array}\right\}+\left[\begin{array}{ll}
\hat{\overline{\mathbf{C}}}_{\mathbf{u u}} & \hat{\mathbf{C}}_{\mathbf{u P}} \\
\hat{\mathbf{C}}_{\mathbf{P u}} & \hat{\mathbf{C}}_{\mathbf{P P}}
\end{array}\right]\left\{\begin{array}{c}
\hat{\mathbf{u}}_{t 2}-\hat{\mathbf{u}}_{t 1} \\
\hat{\mathbf{p}}_{t 2}-\hat{\mathbf{p}}_{t 1}
\end{array}\right\}=\left\{\begin{array}{c}
\hat{\mathbf{F}}_{t 2}-\hat{\mathbf{F}}_{t 1} \\
\Delta t \hat{\mathbf{Q}}(t)
\end{array}\right\}
$$

where

$$
\hat{\overline{\mathbf{p}}}=\varsigma \hat{\mathbf{p}}_{t 2}+(1-\varsigma) \hat{\mathbf{p}}_{t 1}
$$

and $\hat{\overline{\mathbf{C}}}_{\mathbf{u u}}$ and $\hat{\overline{\mathbf{k}}}_{\mathbf{P P}}$ are average values over the integration interval. In Equation (59) $\zeta$ is the integration parameter with a value between 0 and 1 . In differential form the final equation is: 


$$
\left[\begin{array}{cc}
\hat{\mathbf{C}}_{\mathbf{u u}} & \hat{\mathbf{C}}_{\mathbf{u P}} \\
\overline{\mathbf{C}}_{\mathbf{P u}} & -\zeta \Delta t \hat{\mathbf{k}}_{\mathbf{P P}}-\hat{\mathbf{C}}_{\mathbf{P P}}
\end{array}\right]\left\{\begin{array}{c}
\Delta \hat{\mathbf{u}}^{t} \\
\Delta \hat{\mathbf{p}}^{t}
\end{array}\right\}=\left\{\begin{array}{c}
\hat{\mathbf{F}}^{t} \\
\hat{\mathbf{k}}_{\mathbf{P P}} \hat{\mathbf{p}}^{t-1} \Delta t+\hat{\mathbf{Q}}(t) \Delta t
\end{array}\right\}
$$

The time marching solution technique for linear hydroelasticity is unconditionally stable for $\zeta \geq 0.5$, though an early-time solution may exhibit a decaying oscillatory behavior for arbitrary initial time step sizes. To reduce the oscillatory behavior, a predictor-corrector scheme is used in which a solution is sought at $t 1+\theta \Delta t$ and linearly interpolated to $t 1+$ $\Delta t=t 2$. For $\theta=1$ and $\zeta=0.5$ the Crank-Nicholson approximation is obtained. The solution of Equation (60) is obtained by a Newton-Raphson Scheme to allow for material nonlinearity in the stiffness and fluid conductivity matrices (Noorishad et al. 1992).

\section{Extension of formulation for fractured media}

Macroscopic hydromechanical behavior of a fracture under normal closure is governed by a condition of total stress balance between the upper and lower fracture surfaces, and Biot's equations. Biot's equation extended to mechanical behavior of a joint can be written as:

$$
\left\{\begin{array}{l}
d \sigma_{n} \\
d \sigma_{s}
\end{array}\right\}=\left[\begin{array}{cc}
k_{n} & 0 \\
0 & k_{s}
\end{array}\right]\left\{\begin{array}{l}
d u_{n} \\
d u_{s}
\end{array}\right\}-\alpha\left\{\begin{array}{l}
1 \\
0
\end{array}\right\} d p
$$

or which in a compacted form is

$$
d \mathbf{\sigma}^{f}=\mathbf{k}^{f} d \mathbf{u}^{f}-\alpha \mathbf{1}^{f} d p
$$

Note that in Equation (61) $k_{n} \Delta u_{n}$ is the effective normal stress and fluid pressure plays no role in the elastic incremental shear behavior. Similarly to Equation (52), a fracture stress-compatibility equation can be derived from Equation (62) and a cross-fracture total stress balance requirement as: 


$$
\nabla \cdot\left(\mathbf{k}^{f} \frac{\partial \mathbf{u}^{f}}{\partial t}-\alpha \mathbf{1} \frac{\partial p}{\partial t}\right)=\frac{\partial \mathbf{F}}{\partial t}^{f}
$$

Biot's fluid flow equation for a fracture can be written as

$$
\nabla \cdot\left(\frac{b_{h}{ }^{2}}{12 \mu} \nabla(P+\rho g z)\right)-\frac{\alpha}{b} \frac{\partial u_{n}}{\partial t}-\frac{1}{M} \frac{\partial p}{\partial t}=Q
$$

where fracture permeability is defined as

$$
k=\frac{b_{h}^{2}}{12}
$$

and the volumetric strain is approximated as

$$
\frac{\partial \varepsilon_{v}}{\partial t} \approx \frac{1}{b} \frac{\partial u_{n}}{\partial t}
$$

In fractured porous media, fractures may be represented explicitly in a discrete representation using special joint elements (e.g., Goodman 1976) while the matrix rock between fractures is represented by solid elements. The contribution from both solid elements and joint elements are superimposed in the element assembly process. In the case of a joint element, the coefficient matrices in Equation (60) includes the material parameters given in Equations (63) and (64) and appropriate interpolation functions for joint elements. However, for analysis of a large-scale fractured rock mass, meshing of discrete fractures is cumbersome and an equivalent-continuum representation of the fractured rock mass is often the most practical approach. Various approaches for equivalent continuum representation of fractured porous rocks are presented in the next section. 


\section{Equivalent continuum models of HM behavior in fractured rocks}

Equivalent-continuum models for HM behavior of fractured rock masses can be derived using the existing approaches for equivalent representation in hydrogeology or reservoir engineering and in rock mechanics. In rock mechanics, ubiquitously fractured models have been developed in which ideal sets of fractures are considered with their spacing, orientation, and deformation properties. One of the simplest approaches is to assume three orthogonal sets of fractures in a so-called "sugar cube" medium model. Using such an approach, simple equations for the fluid flow and elastic properties can be derived. For example, consider an ideal medium with three sets of parallel fractures in which all sets have the same spacing and the same hydraulic aperture. The permeability along the direction of each set is (Snow 1965)

$$
k=\frac{b_{h}{ }^{3}}{6 s}
$$

where $s$ is fracture spacing. From this equation, it is clear that the fracture aperture is much more important than the fracture spacing for permeability. Likewise, an equivalent compliance for coupling normal stress and normal strain in each direction can be derived as (Gerrard 1982)

$$
\frac{1}{E_{R M}}=\frac{1}{E_{R}}+\frac{1}{k_{n} s}
$$

where $E_{R M}$ and $E_{R}$ are the moduli of deformation for the rock mass and the rock matrix, respectively, and $k_{n}$ is the fracture normal stiffness. Models for orthogonal anisotropic solids (orthotropy) can be used to derive the coefficients in the D-matrix of Equation (50) according to Gerrard (1982). Coupling between fracture deformation and fluid flow can be provided using Biot's equations extended to fractures as in Equations (61) and (64). 
Liu et al. (1999) applied the conceptual model of three orthogonal fracture sets to calculate changes in permeability from normal and shear strains. The sensitivity of permeability to stress in their model is controlled by the initial permeability and the modulus of reduction ratio, $R_{m}=E_{R M} / E_{R}$, a parameter that can be related to the empirical rock mass quality index RMR (Liu et al. 1999).

For non-orthogonal fracture sets, coupled HM behavior can be modeled using a multilaminate model by Zienkiewics and Pande (1977), in which nonlinearities such as sliding and separation of joints can be considered. Using such a model, a permeability tensor may be derived according to Snow (1969).

If fractures are not grouped in sets, but rather randomly distributed, the equivalent properties may be calculated by the concept of the "fabric" or "crack" tensor proposed by Oda (1982). The crack tensor is a unique measure combining four significant aspects of hydraulic and mechanical behavior in fractured rocks: volume, fracture size, fracture orientation and fracture aperture. However, as in any continuum model, a sufficient number of fractures must be included in the volume in which the average properties are calculated to make the derived equivalent properties meaningful (Stietel et al. 1996).

In many cases in reservoir engineering, the storage of fluids is dominated by storage in the matrix, whereas permeability is dominated by fluid flow in fractures. In such a case, so called dual-porosity or overlapping-continua models are applicable. Dual-porosity models were first developed in reservoir engineering in the 1960s (e.g., Barenblatt et al. 
1960). Models incorporating both Biot's poroelastic theory and Bareblatt et al.'s dualporosity concept have been studied by several authors since the 1970s. Recent developments include Chen and Teufel (1997) and Bai et al. (1999).

\section{APPLICATIONS}

This section surveys and new applications where HM coupling is considered significant and HM analysis is performed. The three main fields in which coupled HM analysis is used today are oil and gas explorations, analyses of nuclear waste disposal, and geothermal energy (hot-dry rock) extraction. Other applications where HM coupling is important include coal mining and coal methane extraction, deep-well injection of liquid, and underground storage of natural gas. Relatively new applications include deep injection of solid waste and geogical sequestration of greenhouse gases $\left(\mathrm{CO}_{2}\right)$. This section provides a brief overview of each of these applications with regard to the role of HM coupling.

\section{Hydrogeology and well test analysis}

Coupled HM effects are generally not considered in conventional geohydrology and well test analysis except through the concept of specific storage. It is well known that the classical solutions by Jacob (1940) and the concept of coefficient of storage in Equation (4) above is based on a one-dimensional mechanical model and a two- or threedimensional flow geometry. The partially coupled Jacob diffusion equation can be derived from Biot's fully coupled equations. First, a complete flow equation for a fully 
coupled system can be derived by combining Darcy's law with Equations (9) and (24), leading to:

$$
\nabla \cdot\left(\frac{\mathbf{k}}{\mu_{f}} \nabla\left(p+\rho_{f} g z\right)\right)=\alpha \frac{\partial \varepsilon_{v}}{\partial t}+\left(\frac{\phi}{K_{f}}+\frac{(\phi-\alpha)}{K_{s}}\right) \frac{\partial p}{\partial t}
$$

This is a fully coupled equation, since fluid flow depends on mechanical deformation, through the volumetric strain. In an uncoupled (Jacob's) approach, the volumetric strain in Equation (69) is calculated with the assumption of no lateral strain and a constant total vertical stress, according to

$$
\alpha \frac{\partial \varepsilon_{v}}{\partial t}=\alpha \frac{\partial \varepsilon_{z}}{\partial t}=\frac{\alpha}{K_{v}} \frac{\partial \sigma_{z}^{\prime}}{\partial t}=\frac{\alpha^{2}}{K_{v}} \frac{\partial p}{\partial t}
$$

and the uncoupled flow equation can be written as

$$
\nabla \cdot\left(\frac{\mathbf{k}}{\mu_{f}} \nabla\left(p+\rho_{f} g z\right)\right)=\left(\frac{\phi}{K_{f}}+\frac{(\phi-\alpha)}{K_{s}}+\frac{\alpha^{2}}{K_{v}}\right) \frac{\partial p}{\partial t}
$$

where $K_{v}$ is uniaxial (vertical) deformation modulus. The coefficient

$$
S_{s 1}=\frac{\phi}{K_{f}}+\frac{(\phi-\alpha)}{K_{s}}+\frac{\alpha^{2}}{K_{v}}
$$

is the specific storage, which for incompressible grains $\left(\alpha=1\right.$, and $\left.K_{s}=\infty\right)$ reduces to the usual Jacob specific storage coefficient, $S_{s}$, defined in Equation (7). Then, by substituting $p=(h-z) \rho g$ into Equation (71), the exact Jacob diffusion Equation (6) can be derived.

Most well-test formulas are based on a partially coupled diffusion equation, with the assumption of a constant vertical stress for the derivation of specific storage and of a constant permeability that does not change with injection pressure. The assumption of 
constant permeability could present difficulties in fractured rocks, where the permeability can be very sensitive to injection pressure. Figure 21 presents a recent example from a test on fractures in granite located at $70 \mathrm{~m}$ depth. The injection pressure is $1.2 \mathrm{MPa}$, which is well below the total normal stress across the horizontal fracture, estimated at 2.0 MPa. The flow rate first stays roughly constant for about 15 minutes. Thereafter, the fracture starts to jack open, and the flow increases with time to reach a maximum injection rate after 60 minutes. Later, the flow rate decreases again probably because it has been completely filled with pressurized water as shown in the lower part of Figure 21. This exemplifies that permeability is not constant, but rather changes with pressure inside the fracture. However, the test is conducted at shallow depths and the fluid pressure is only $0.8 \mathrm{MPa}$ below the lithostatic stress. In most cases, a fluid pressure well below $20 \%$ of the lithostatic stress is sufficiently low to avoid an increasing permeability. A hydraulic jacking test as previously described can be conducted to investigate whether the injection pressure is sufficiently low to avoid significant changes in permeability.

Coupled HM analysis of injection tests can be used to constrain subsurface hydraulic properties. The mechanical responses of fractures dominate the storativity during a hydraulic injection test in low permeability fractured rocks. Local and regional stress balance in a rock mass and its mechanical stiffness provide a confinement that restricts mechanical movements within certain limits. This means that the mechanics of the rock and rock fractures provide practical bounds for fracture storativity and specific storage. As demonstrated by Rutqvist (1995c), this mechanism can be used to constrain fracture storativity when interpreting well tests. 


\section{Oil and gas extraction}

HM coupling is important for a multitude of problems related to oil and gas exploration and extraction, including poroelastic stress redistribution around a borehole, reservoir compaction and subsidence, as well as the mechanics of hydraulic fracturing (Geertsma 1957, 1966, 1973). Since the 1970s, hydraulic fracturing technology has been the most important topic for HM coupling in oil and gas exploration. Recently, other areas have attracted interest: borehole stability and shale mechanics, reservoir compaction and subsidence, enhanced oil recovery (Dusseault 1997), and stress-sensitive permeability changes during production (Davies and Davies 1999). Reservoir compaction is nowadays generally analyzed using partially or fully coupled numerical modeling approaches; see the paper by Lewis et al. (2003) in this Special Issue. In a partially coupled approach, the pressure pattern obtained from reservoir simulators is used in a stress-strain analysis to compute the compaction of the reservoir and the subsidence of the land surface (Gambolati and Freeze 1973 and Gambolati et al. 1991). However, fully coupled models based on Biot's theory of consolidation have also been used (e.g., Lewis and Schrefler 1998; Gutierrez et al. 1995). Recently, several fully coupled reservoir simulation models, with the capability of simulating stress dependent permeability, have been developed for oil and gas production simulations (e.g., Lewis and Sukirman 1993; Koutsabeloulis and Hope 1998; Gutierrez and Lewis 1998; Osoiro et al. 1998). Such models might be increasingly important as conventional oil supplies are depleted. Extraction of unconventional heavy oil requires thermal recovery methods or other techniques that involve massive stress changes coupled with fluid flow and evolution of mechanical 
properties (Dusseault 1997). Thus, analysis of coupled thermo-hydromechanical (THM) processes will be increasingly important for oil and gas exploration.

\section{Geothermal energy}

Several large projects, directed at establishing the technology for developing commercial engineered geothermal systems are now under way in various parts of the world. At the time of the first "hot dry rock" (HDR) project at Fenton Hill in the USA, discussion was mainly focused on a subsurface reservoir system consisting of one or more simple artificial penny-shaped fractures propagating in the direction of the maximum principal stress (Figure 22a). The term "dry" in HDR came from the fact that the intact basement granites where the tests were performed were of very low permeability. Once HDR reservoirs were recognized as essentially manmade variants of natural hydrothermal reservoirs the similarities between HDR hydrothermal resources and extractions from natural systems became apparent.

In the early 1980s, Pine and Batchelor (1984) confirmed that the creation of new fractures was not the dominant process during the injection of water into the rock mass at great depth. Far more important was the shearing of natural joints and in particular those aligned with the principal stresses of the local stress field as shown in Figure 22b. As previously discussed the joints fail in shear because the fluid injection reduces the normal stress across them and allows frictional slippage to occur before jacking. This was first demonstrated for the Cornwall HDR project in the Carnmenellis granite where injection was conducted at depths greater than $2 \mathrm{~km}$ below ground level. Microseismic events 
detected during the high flow rate stimulations indicated strike-slip shear consistent with the orientation of the natural joints and in situ stress conditions. The events were located between about $500 \mathrm{~m}$ above and $2 \mathrm{~km}$ below the point of hydraulic injection and they were used for the location of the HWR reservoir. Later stimulations using gel and water gave improved connectivity between the boreholes drilled into the reservoir and reduced circulation impedance.

In the 1980s the term "hot wet rock" (HWR) system was proposed for geothermal extraction systems in which hydraulic fracturing and injection were used for permeability enhancements and for sustaining production in naturally fractured hot rock masses that exhibit only minor flow. In Europe the HWR system is sometimes called the "Graben Concept" as it is associated with areas of graben structure where stresses tend to be low, which allows stimulation and circulation to be carried out at moderate pressures (Baria et al. 1999a). The concepts that have evolved in the last 25 years for HDR/HWR are shown in Figure 22. A summary of the most important HDR/HWR field experiments and their target temperatures, regional and local geology, and geochemistry was presented by Nakatsuka (1999), and the relevant stress and rock mechanics issues by Evans et al. (1999).

In the last eight years or so the concept of HDR/WDR has shifted again to take greater benefit of the geological environment. Hence, at the European Community project at Soultz-sous-Forets, the reservoir is located in a typical graben structure, where high temperature can be encountered at shallow depth at relatively low minimum stress 
gradients, in which faults or fault zones are already partially open for flow. At Soultz, a system consisting of two production wells and one injection well is planned, in which the three boreholes are likely to be aligned parallel with the direction of the maximum horizontal stress, with a smaller normal stress on fractures connecting the wells (Baria et al. 1999b). In Sweden, the concepts will be applied to areas with low heat flow. In the county of Scania in southernmost Sweden, at the edge of the Baltic Shield, a well is being drilled to target one of the major fault zones in Precambrian granites and gneisses at about $4 \mathrm{~km}$ depth. Warm water (estimated at $70^{\circ} \mathrm{C}$ ) will be extracted from the fault and later used for heating in the city of Lund. Another project at Björkö in central Sweden is located in a $10 \mathrm{~km}$-diameter meteorite impact crater of Precambrium age about $20 \mathrm{~km}$ west of Stockholm (Henkel 2002). The impact has caused severe fracturing of the bedrock, with fractures later healed from calcite, chlorite and laumontite precipitation. These are low-strength minerals, and the present concept is to stimulate and reopen existing fractures and create a highly permeable, low temperature geothermal reservoir. After heat exchange at the production hole, the warm water will be fed into the existing district heating system of Stockholm.

\section{Nuclear waste disposal}

In general, HM coupling is part of the coupled thermo-hydromechanico-chemical (THMC) processes that are being studies as part of the performance assessment for nuclear waste repositories. Coupled thermo-hydro-mechanical (THM) processes are important because the heat released from the spent fuel will heat the surrounding rock mass, creating thermal stresses. Depending on repository design and waste 
characteristics, the temperature may reach a maximum of 50 to $150{ }^{\circ} \mathrm{C}$, with the greatest thermo-mechanical impact occurring around 100 to 1,000 years after waste emplacement. There are two main areas in which HM coupling could have a significant impact on a repository performance: (1) the mechanical integrity of the repository and (2) possible changes in radionuclide transport properties. HM coupling affects the mechanical integrity and stability of the repository vaults through the effective stress law. This effect must be predicted throughout the repository lifetime because the fluid pressure (in saturated rock formations) will generally increase over time because of restoration of hydrostatic fluid pressure that was drained during the construction phase. Transport properties may change as a result of the excavation in the EDZ. Furthermore, during heating of the rock mass, thermal stresses can change fracture apertures through thermoelastic closure, opening or shear slip, leading to permeability changes that may or may not be irreversible after the period of heating.

Prediction of coupled THM processes associated with nuclear waste repositories thousands of years in the future requires robust models and these models must be supplied with realistic input data. These issues were first discussed in a series of workshops and international symposiums at the Lawrence Berkeley National Laboratory, U.S.A. in the early and mid-1980s (Tsang 1987). In 1991, an international collaboration project DECOVALEX (DEvelopment of COupled MOdels and their VALidation against EXperiments in nuclear waste isolation) were launched. The project, and its followups DECOVALEX II and III, have been sponsored by nuclear waste agencies in nine countries and have involved some 20 research organizations (Stephansson et al. 1996). 
Within the project, the different research teams are assigned to use their respective numerical models to predict coupled THM responses in well-defined hypothetical bench mark tests (BMTs) and test cases (TCs) of actual experiments. The tasks have involved coupled THM problems from the scale of laboratory samples to kilometers. A number of major field experiments have been TCs in DECOVALEX, e.g., Fanay Augères in France (Rejeb et al. 1996), Kamaishi Mine, Japan (Rutqvist et al. 2002), FEBEX at Grimsel in Switzerland, and the Drift Scale Test (DST) at Yucca Mountain, Nevada. HM coupling is part of all these experiments. However, direct measurements of permeability changes during heating have only been conducted in the recent Drift Scale heater Test at Yucca Mountain. This ongoing experiment indicates that permeability decreases up to one order of magnitude during heating because of thermal stress and associated closure of rock fractures. Such data was used by Rutqvist and Tsang (2002) to back-calculate in situ HM properties of the fractured rock at Yucca Mountain. These in situ calibrated properties were then used to predict coupled THM effects at a potential repository at Yucca Mountain and estimate the impact of HM coupling on the long-term performance of the repository (Rutqvist and Tsang 2002).

\section{Deep well injection of liquid waste}

The possibility of induced earthquakes was mentioned previously in connection with the waste injection operation at the Rocky Mountain Arsenal near Denver, in the early 1960s. However, while the injection-induced seismicity is of great concern, it is a rare phenomenon that does not pose a problem for the vast majority of active injection wells. Instead, it is the potential for contaminating otherwise usable groundwater that is of most 
concern (Apps and Tsang 1996). A breech in the confining interval present above the injection zone is one possible avenue of fluid migration from the injection zone. Such fluid migration can be enhanced if the confining layer is intersected by natural faults and fractures, or by an induced fracture. However, since the 1980s, more stringent regulation for deep-well injection in the U.S. requires comprehensive HM characterization of injection zones and confining layers before and during injection operations. Regulations in different states usually require the maximum injection pressure to be set below the fracture pressure of the injection zone, to ensure that the confining zone does not fracture. During an injection operation, injection pressure, injection volume, and flow rate must be continuously monitored, because any change in the relationship between the variables could indicate downhole problems. In addition, yearly fall-off pressure tests are required to investigate possible changes in the geometry of stimulated fractures in the injection zone (Brasier and Kobelsky 1996).

\section{Deep well injection of solid waste}

The use of hydraulic fracturing for disposal of drill cuttings and other wastes, or so-called Slurry Fracture Injection (SFI), has become more widely used in the last decade as an alternative to traditional landfill disposal for oilfield wastes. Large-scale operations have taken place in the Gulf of Mexico (Reed et al. 2001), Canada (Dusseault et al. 1996), Alaska (Schmidt et al. 1999), California (Hainey et al. 1997) and the North Sea. SFI is conducted by mixing a slurry of solids and fresh or produced water and injecting it at high pressure into suitable sand formations contained within layers of low-permeability shales. The low-permeability shales should act as a fracture barrier to blunt upward 
fracture growth and prevent vertical fluid communication. Whether the low-permeability formation adjacent to the injection zone will act as a fracture barrier is strongly dependent on the stress contrast between the layers (Nolte 1991). During a slurry injection, the carrying fluid bleeds off rapidly, leaving behind solid waste within the fracture. An injection operation over 24 hours frequently involves an injection episode and a relaxation episode. The relaxation period is necessary to relax fluid pressure and poroelastic stress buildup in the rock mass surrounding the fracture, which otherwise would close the fracture and reduce injectivity. Because the injection takes place at a pressure above fracturing pressure, a careful hydromechanical characterization of the injection interval and the caprock, as well as monitoring of the fracture geometry, is essential to ensure that the fracture does not break though the overlying cap. During operation, fracture extension can sometimes be tracked by monitoring of acoustic emission and surface tiltmeters (Withers et al. 1996).

\section{Coal mining and coal methane extraction}

Coal is generally characterized by a dual porosity, containing both micropore and macropore systems. The macropore system consists of a naturally occurring network of fractures called the cleat system, with apertures varying from 100 to $0.1 \mathrm{~nm}$ (Harpalani and Chen 1995). Because of the cleat structure, the permeability of coal has shown to be extremely sensitive to stress changes and gas pressure. In addition, the release of methane gas from coal induces shrinkage in the matrix, which also strongly affects the permeability of the cleat system. These HM coupled processes can have important consequences during underground mining of coal (Liu and Elsworth 1997, Xiao and Xu, 
2000) and during extraction of methane from coal seams. To recover a large percentage of methane gas in coalbeds, reservoir pressure must be reduced significantly (Harpalani and Schraufnagel 1990). Two distinct effects are associated with the reduction of pressure during methane extraction: (1) the release of gas and (2) an increase in effective stress. The increase in effective stress causes a decrease in the permeability of the coal owing to mechanical closure of flow paths. However, an opposing effect of coal-matrix shrinkage can occur, one that is attributed to a desorbtion phenomenon. This shrinkage widens the fractures that are primarily responsible for gas flow in coalbed reservoirs, and thus increases permeability. These effects occur simultaneously and, depending on in situ conditions, the permeability can decrease or increase during production. A coupled HM analysis of a coal-methane extraction operation seems to be essential for optimizing the production.

\subsection{Geological storage natural gas}

Underground gas storage in reservoirs and rock caverns is a mature technology that has been practiced for decades. In North America, it is a typical practice to operate gasstorage reservoirs at or below the original reservoir pressure, out of concerns about caprock integrity, fracturing, faulting and gas loss. As pointed out by Bruno et al. (1998), the maximum safe operating pressure depends on several geomechanical factors, including in situ stresses, stresses induced by local and global changes in the reservoir, and the mechanical and hydraulic properties of the reservoir and overburden. Nagelhout and Roest (1997) presented numerical modeling of a generic underground natural gas storage facility to investigate the possibilities of fault slip in some of the new gas storage 
facilities in The Netherlands. The result of Nagelhout and Roest's (1997) simulation showed that fault slip of up to $5 \mathrm{~cm}$ occurred during the initial depletion of fluid pressure from 300 bar to 115 bar. On the other hand, no inelastic fault slip occurred during the following simulated gas storage operations. However, the fluid pressure did not exceed the initial reservoir pressure by more than $14 \%$.

\section{Geological sequestration of $\mathrm{CO}_{2}$}

Recently, underground storage of carbon dioxide $\left(\mathrm{CO}_{2}\right)$ into permeable aquifers, such as deep saline aquifers, depleted oil and gas reservoirs, and coal seams, has been suggested as an important potential method for reducing the emission of greenhouse gases to the atmosphere (DOE 1999). The injection would take place at a depth below $800 \mathrm{~m}$ so that the $\mathrm{CO}_{2}$ would be within the temperature and pressure range of a supercritical fluid. As a supercritical fluid, $\mathrm{CO}_{2}$ behaves like a gas with low viscosity but with a liquid-like density of $200-900 \mathrm{~kg} / \mathrm{m}^{3}$, depending on pressure and temperature. Because supercritical $\mathrm{CO}_{2}$ is less dense than water, deep underground disposal requires a sufficiently impermeable seal (caprock) above an underground storage zone to trap the injected $\mathrm{CO}_{2}$. A caprock may be discontinuous and may contain imperfections such as faults and fractures of various sizes (from small meter-scale fractures to kilometer-scale faults). Furthermore, the hydraulic properties of a fault may change with injection-induced hydraulic pressure. Thus, the performance assessment of $\mathrm{CO}_{2}$ storage in an underground brine formation requires coupled HM analysis, including multiphase flow and failure analysis. A first study of coupled $\mathrm{HM}$ numerical modeling of the effects during $\mathrm{CO}_{2}$ injection into a brine formation has recently been conducted by Rutqvist and Tsang 
(2002). The simulation is conducted as a linked TOUGH2 - FLAC3D simulation, in which TOUGH2 is a reservoir simulator for multiphase flow and heat transport and FLAC3D is a rock mechanics code (Rutqvist et al. 2002).

\section{CONCLUDING REMARKS}

Based on laboratory and field data reviewed in this article, a few general observations can be made. First of all, indirect coupled processes (for example stress-dependent permeability) tend to be most pronounced in intact rocks containing flat microcracks, such as shale and granite, and in rocks containing macrofractures. The sensitivity of permeability to stress in fractured rock depends on hydraulic properties, such as fracture permeability and interconnectivity of the conducting fracture network, and on mechanical parameters, such as fracture normal stiffness and fracture shear strength. In general, the most important parameters for the stress-permeability relationship of a single connected fracture are:

- Initial permeability

- Initial fracture normal stiffness

- Fracture shear strength

All three of these parameters depend on the initial effective stress normal to the fracture. However, while initial fracture normal stiffness and fracture shear strength can be well correlated to the virgin in situ stress, no such clear correlation between permeability and the virgin stress exists. This is because permeability is sensitive to the effects of mineral filling (see Figure 11). At one extreme, mineral filling can completely block the fluid 
flow in a fracture. At the other extreme, hard mineral filling can keep the fracture open, creating large flow channels between rock bridges in the fracture plane.

Many field experiments have shown that, in general, fractures with a large initial permeability are less sensitive to changes in effective normal stress than fractures with a small initial permeability. Hydraulic borehole tests show that relatively large open-flow channels can exist at depths of several thousand meters. These fractures are so conductive that they are likely to be locked open either by large shear displacement and/or hard mineral filling. These highly permeable fractures will tend to be relatively stressinsensitive because their stiffness is relatively high. The fact that these features stay open indicates that they consist of low-aspect-ratio features which, according to Equation (16), could stay open in very high stress environments. Another extreme is fractures that are shallow and partially filled with soft-mineral filling. Shallow mineral filled fractures have a relatively low fracture normal stiffness, and at the same time a small initial hydraulic aperture because the fracture surfaces are coated with mineral filling. Such fractures can be extremely sensitive to changes in stress and fluid pressure.

A fracture with a large initial permeability is also likely to be less sensitive to shear displacements. There are several reasons for this. First, a large initial aperture is likely found in large (long) fractures (see Figure 5), which for a given shear displacement do not dilate as much as a small fractures (see Figure $4 c$ and $d$ ). Second, a large initial aperture is likely to be found in a fracture that has already been sheared. For example, the fracture depicted in Figure 8 has experienced a shear displacement of centimeters. It has 
already been sheared passed its peak shear strengths, which means that the shear dilation curves are flat and that any further shear may give relatively small changes in permeability (see Figure 4d). However, if the initial permeability is small, it can indicate that it is a mated joint without previous shear, or it could be a fault that is almost completely mineral filled. In the case of a mineral-filled fracture, any shearing could induce extreme changes in permeability through breakdown of seals.

Fractured rock permeability at depth is likely to be less sensitive to a disturbance (e.g. underground construction) than fractured rocks at shallow depths (as illustrated in Figure 23). This has been confirmed at large scale field tests in fractured rocks. For example, at the Yucca Mountain site, Nevada, U.S.A., the initial horizontal stresses are very low, on the order of $2 \mathrm{MPa}$. This implies that fractures are open much more than their residual values and that the initial fracture normal stiffness is relatively low. Results from field tests at Yucca Mountain indicate that rock mass permeability can be reduced one order of magnitude by stress increases during large-scale heater tests, before reaching a residual permeability value. Tests of excavation disturbed zones at other sites where the initial stress is higher indicates little change in permeability outside the damaged zone. One reason might be that permeability of fractures tends to be close to their residual values at depths, which implies that any further stress increase will not produce any further fracture closure (Figure 23). On the other hand, a reduced stress, for example from unloading normal to a drift wall, can produce an opening of fractures at any depth. 
Pressure-induced changes during injection are also likely to be more pronounced at shallow depth. One example is the injection test presented in Figure 21, showing an increasing fracture transmissivity with fracture fluid pressure. In this case an injection pressure of $1 \mathrm{MPa}$ above the initial pressure gives significant changes in fracture permeability. In the example of the hydraulic jacking test in Figure $9 c$, conducted at a depth of about $300 \mathrm{~m}$, an injection pressure of $1 \mathrm{MPa}$ over the initial gives no significant changes in permeability. Injection-induced fracture shear also tends to be more likely in shallow areas, because the in situ stress field can be more anisotropic, and at the same time the shear strength is small because of the relatively small effective stress.

Figure 23 shows a generally reduced stress dependency for permeability with depth. However, fracture permeabilities at great depth can be highly stress dependent if their initial values are low in relation to their initial normal stiffness. As seen in the conceptual model for fractured rocks in Figure 6, there are clusters with highly permeable fractures, which are connected by sections containing much less permeable fractures. These low permeability bridges of more competent rock might control the overall kilometer-scale flow through the rock mass. Since these bridges have a lower permeability, they are also likely to have a more stress-dependent permeability than the more permeable fracture clusters.

To be able to estimate the range of possible HM responses to a human-induced disturbance (e.g., massive injection or underground construction), an in situ testing program is recommended. If $\mathrm{HM}$ interactions are significant for a certain application (i.e., 
geological sequestration of $\mathrm{CO}_{2}$ ) then in situ hydromechanical characterization should be carried out routinely, just as hydraulic permeability tests are done. This includes a complete determination of the in situ stress field followed by in situ tests to back-analyze the stress-permeability relationship of the fractured media.

Although coupled numerical modeling has advanced greatly over the past 30 years, applying numerical modeling to coupled HM analysis can lead to great uncertainties. Many of these uncertainties stem from the inability to characterize heterogeneous fractured rock masses and to capture all critical material features in a numerical model. In fractured rock, it might be possible to predict the general responses, but on a local scale it can be difficult to predict responses other than probabilistically. In a sparsely fractured rock mass, one critical discrete fracture, connected to a network of conducting fractures, can have substantial effects on permeability depending on its location and orientation. Such a situation is difficult to predict, because the precise locations of such fractures are generally not known in advance.

\section{Acknowledgements}

Technical review and comments by Dr. Christopher E Neuzil, U.S. Geological Survey; Dr. Chin-Fu Tsang, Lawrence Berkeley National Laboratory and Tech Lic. KiBok Min, Royal Institute of Technology, Sweden are much appreciated. The following organizations are gratefully acknowledged for their financial support: the Director, Office of Science, Office of Basic Energy Sciences, Division of Chemical Sciences, Geosciences and Biological Sciences, of the U.S. Department of Energy, under contract 
No. DE-AC03-76-SF00098; the DECOVALEX Project through the Swedish Nuclear

Power Inspectorate; and the European Commission through the BENCHPAR project under Contract FIKW-CT-2000-00066. 


\section{NOTATIONS}

Most symbols are defined the first time they occur. The following list contains an explanation of the symbols that needs further explanation and symbols that are most frequently used. Boldface letters represent matrix or vector quantities, and scalars are printed in italic.

$$
\begin{aligned}
& A \quad=\quad \text { Area }\left[\mathrm{m}^{2}\right] \\
& b \quad=\quad \text { Physical fracture aperture }[\mathrm{m}] \\
& b_{E}=\quad \text { Barton et al.'s (1985) "real” physical aperture (Equation (42)) [m] } \\
& b_{h} \quad=\quad \text { Hydraulic conducting aperture Equation (26) [m] } \\
& C \quad=\quad \text { Flow geometry constant }\left(=\rho_{f} g w / 12 \mu_{f}\right. \text { for parallel flow, Equation (13)) [-] } \\
& \hat{\mathbf{C}}_{\mathbf{u u}}=\quad \text { FEM incremental stiffness matrix }[\mathrm{Pa}] \\
& \hat{\mathbf{C}}_{\mathbf{u P}}=\quad \text { FEM coupling matrix }[-] \\
& \hat{\mathbf{C}}_{\mathbf{P u}}=\hat{\mathbf{C}}_{\mathbf{u P}}^{t r} \\
& \hat{\mathbf{C}}_{\mathbf{P P}}=\quad \text { FEM fluid storage matrix } \\
& \text { D } \quad=\quad \text { Tensor of elastic constants }[\mathrm{Pa}] \\
& E \quad=\quad \text { Young's modulus }[\mathrm{Pa}] \\
& f \quad=\quad \text { A friction factor for fluid flow in a rough fracture (Equation (36)) [-] } \\
& \mathbf{F}=\text { Mechanical force vector }\left(=\left\{\mathrm{F}_{\mathrm{x}}, \mathrm{F}_{\mathrm{y}}, \mathrm{F}_{\mathrm{z}}\right\}^{\text {tr }} \text { in Cartesian coordinates }\right) \\
& \hat{\mathbf{F}}=\text { FEM nodal force vector } \\
& g=\quad \text { Acceleration of gravity }\left[\mathrm{m} / \mathrm{s}^{2}\right] \\
& G \quad=\quad \text { Shear modulus }[\mathrm{Pa}]
\end{aligned}
$$




\begin{tabular}{|c|c|c|}
\hline$h$ & $=$ & Total hydraulic head $\left(=p / \rho_{f} g+z\right)[\mathrm{m}]$ \\
\hline I & $=$ & Identity tensor (all components 0 except diagonals which are 1) [-] \\
\hline$J C S$ & $=$ & Joint Compressive Strength (measured by a hammer rebound test) [Pa] \\
\hline$J R C$ & $=$ & Joint Roughness Coefficient (a measure of fracture surface roughness) [-] \\
\hline$J R C_{m o b}$ & & Mobilized $J R C$ (a function of shear displacement) [-] \\
\hline$k$ & $=$ & Permeability $\left[\mathrm{m}^{2}\right]$ \\
\hline$k_{0}$ & $=$ & Permeability at a reference stress $\left[\mathrm{m}^{2}\right]$ \\
\hline$k_{n}$ & $=$ & Fracture normal stiffness (Figure le) $[\mathrm{Pa} / \mathrm{m}]$ \\
\hline$k_{n i}$ & $=$ & Fracture normal stiffness at an initial effective stress (Figure $4 a)[\mathrm{Pa} / \mathrm{m}]$ \\
\hline$k_{n 0}$ & $=$ & Fracture normal stiffness at zero normal stress (Figure $4 a)[\mathrm{Pa} / \mathrm{m}]$ \\
\hline$k_{s}$ & $=$ & Fracture shear stiffness (Figure 1f) $[\mathrm{Pa}]$ \\
\hline $\mathbf{k}$ & $=$ & Permeability tensor $\left[\mathrm{m}^{2}\right]$ \\
\hline$\overline{\mathbf{k}}_{\mathbf{P P}}$ & $=$ & FEM incremental conductance matrix \\
\hline$K$ & $=$ & Drained bulk modulus $[\mathrm{Pa}]$ \\
\hline$K_{f}$ & $=$ & Bulk modulus of pore fluid $[\mathrm{Pa}]$ \\
\hline$K^{\prime}$ & $=$ & Modulus of drained uniaxial (or vertical) porous medium deformation [Pa] \\
\hline$K_{s}$ & $=$ & Bulk modulus of solid grains $[\mathrm{Pa}]$ \\
\hline$M$ & $=$ & Biot's effective isothermal storage constant (Equation (9) and (24)) [Pa] \\
\hline$p$ & $=$ & Fluid pressure $[\mathrm{Pa}]$ \\
\hline$P$ & $=$ & Total isotropic pressure $\left(=\sigma_{x x}+\sigma_{y y}+\sigma_{z z}\right.$, compressive positive $)[\mathrm{Pa}]$ \\
\hline$P^{\prime}$ & $=$ & Effective isotropic pressure $\left(=\sigma_{x x}^{\prime}+\sigma_{y y}^{\prime}+\sigma_{z z}^{\prime}\right.$, compressive positive $)[\mathrm{Pa}]$ \\
\hline$\hat{\mathbf{p}}$ & $=$ & FEM vector of nodal fluid pressure $[\mathrm{Pa}]$ \\
\hline$Q$ & $=$ & Volume flow rate $\left[\mathrm{m}^{3} / \mathrm{s}\right]$ \\
\hline
\end{tabular}




$$
\begin{aligned}
& Q^{f}=\quad \text { Volume flow rate per unit fracture width }\left[\mathrm{m}^{3} / \mathrm{s}\right] \\
& \hat{\mathbf{Q}}=\text { Nodal flow rate vector }\left[\mathrm{m}^{3} / \mathrm{s}\right] \\
& R=\quad \text { Aspect ratio of an elliptical crack (= } b_{c 0} \text { /"crack-length", Equation (16)) [-] } \\
& s \quad=\quad \text { Fracture spacing }[\mathrm{m}] \\
& S, S^{f}=\quad \text { Jacob storage coefficient or storativity (Equation (3) and (25)) [-] } \\
& S_{s} \quad=\quad \text { Jacob specific storage (Equation (7)) }\left[\mathrm{m}^{-1}\right] \\
& t \quad=\quad \text { Time (seconds) } \\
& \operatorname{tr} \quad=\quad \text { See special symbols below } \\
& T=\text { Fluid transmissivity }\left[\mathrm{m}^{2} / \mathrm{s}\right] \\
& \left.T_{r}=\text { Residual transmissivity at high compressive stress (Figure } 4 b\right)\left[\mathrm{m}^{2} / \mathrm{s}\right] \\
& \mathbf{u} \quad=\quad \text { Displacement (vector) }[\mathrm{m}] \\
& \hat{\mathbf{u}}=\text { FEM nodal displacement vector } \\
& u_{n}=\text { Fracture normal displacement (Figure 4a) }[\mathrm{m}] \\
& u_{s}=\quad \text { Fracture shear displacement (Figure 4c) }[\mathrm{m}] \\
& w \quad=\quad \text { Width of a fracture plane }[\mathrm{m}] \\
& z \quad=\quad \text { z-coordinate or elevation }[\mathrm{m}]
\end{aligned}
$$

Greek symbols

$$
\begin{array}{llll}
\alpha & = & \text { Biot-Willis' coefficient (Equation }(10) \text { and }(23))[-] \\
\delta & = & \text { Fracture normal closure }(\text { Figure } 4 a)[\mathrm{m}] \\
\delta_{\max } & = & \text { Maximum fracture normal closure }(\text { Figure } 4 a)[\mathrm{m}] \\
\varepsilon & = & \text { Total strain tensor }[-] \\
\varepsilon_{v} & = & \text { Volumetric strain }\left(=\varepsilon_{\mathrm{xx}}+\varepsilon_{\mathrm{yy}}+\varepsilon_{\mathrm{zz}}, \text { positive for contraction }[-]\right.
\end{array}
$$




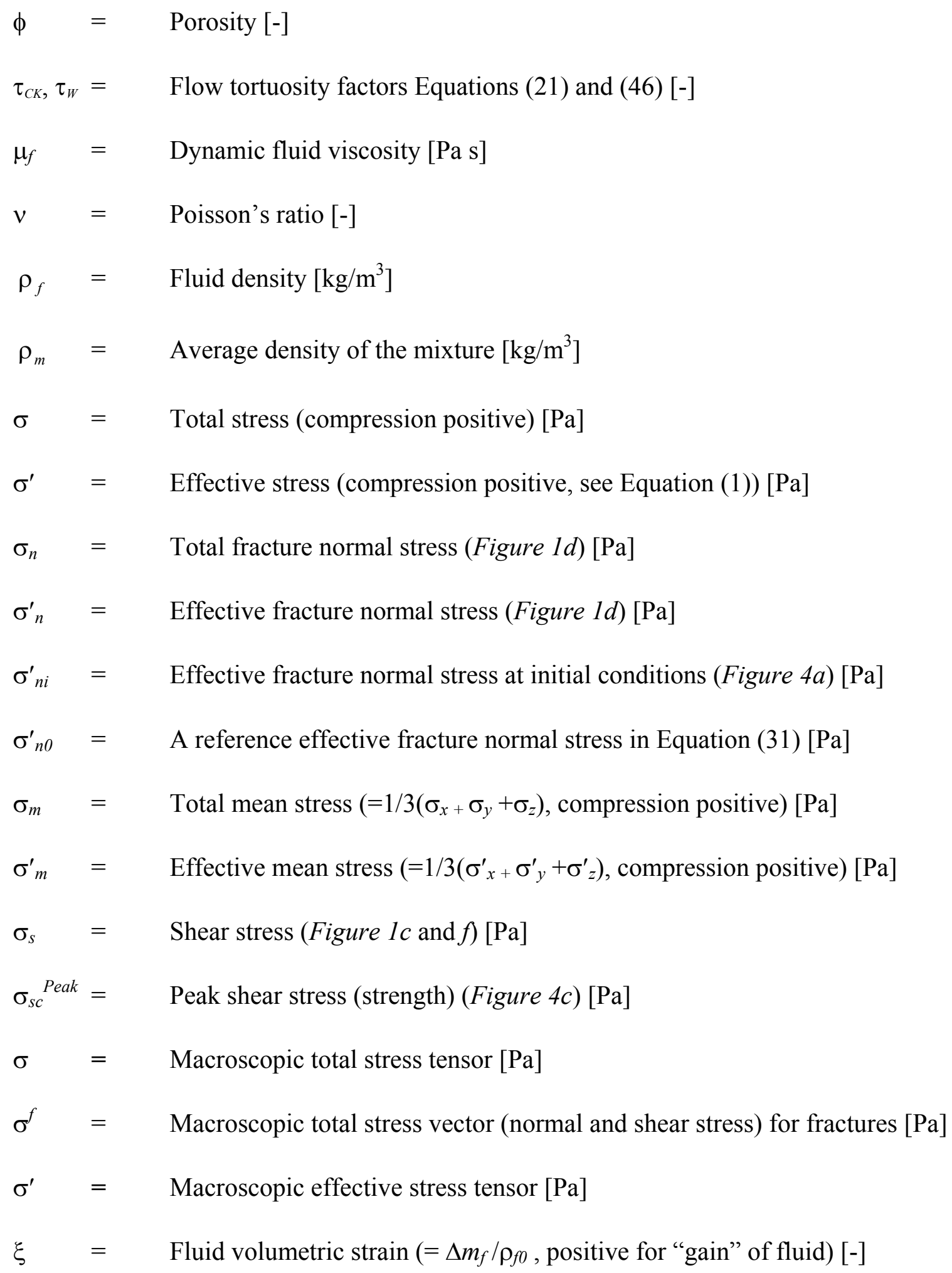



$\mathbf{A}^{\text {tr }}=\quad$ Transpose of a vector
$\nabla \cdot \mathbf{A}=\quad$ Divergence of a vector $(=\operatorname{div} \mathbf{A})$
$\nabla A=\quad$ Gradient of a scalar $(=\operatorname{grad} A)$
$\nabla \mathbf{A}=\quad$ Gradient of a vector
$\mathbf{A}: \mathbf{B}=$ Contracted product of two tensors 


\title{
REFERENCES
}

Aki K, Fehler M, Aamodt R L, Albright J N, Potter R M Pearson C M and Tester J W (1982) Interpreatation of sesimic data from hydraulic fracturing experiments at the Fenton, Hill New Mexico, Hot Dry Rock Geothermal site. J Geophys Res 87:936-951

Alm P (1999) Hydro-mechanical behavior of a pressurized single fracture: An in situ experiment. PhD, Chalmers University, Sweden

\begin{abstract}
Alonso EE, Gens A and Delahaye CH (2003) Influence of rainfall on the deformation and stability of a slope in overconsolidated clays. A case study. This volume
\end{abstract}

Apps J and Tsang C-F (1996) Preface. In: Apps J and Tsang C-F (eds) Deep Injection of Disposal of Hazardous Industrial Waste. Scientific and engineering aspects. Academic Press, San Diego, California

Bai M, Feng F, Elsworth D and Roegiers J-C (1999) Analysis of stress-dependent permeability in nonorthogonal flow and deformation. Rock Mech and Rock Engng $32: 195-219$

Bandis S, Lumsden A C and Barton N R (1983) Fundamentals of rock joint deformation. Int J Rock Mech Min Sci \& Geomech Abstr 20:249-268 
Barenblatt G I, Zheltov I P and Kochina (1960) Basic concepts in the theory of homogenous liquids in fissured rocks Prikl Mat Mekh 24:852-864

Baria R, Baumgärtner J, Rummel F, Pine RJ, Sato Y (1999a) HDR/HWR reservoirs: concepts, understanding and creation. Geothermics 28:533-552

Baria R, Baumgärtner J, Gérard A, Jung R and Garnish J (1999b) European HDR research programme at Soultz-sous-Forets (France) 1987-1996. Geothermics 28 $655-669$

Barton and Choubey (1977) The shear strength of rock joints in theory and practice. Rock Mech 10:1-54

Barton N R and Bakhtar K (1982) Rock joint description and modeling for the hydrothermomechanical design of nuclear waste repositories. Technical Report 83-10, TerraTek Engineering, Salt Lake City, Utah

Barton N R, Bandis S and Bakhtar K (1985) Strength, deformation and conductivity coupling of rock joints. Int J Rock Mech Min Sci \& Geomech Abstr 22:121-140 
Barton C A, Zoback M D and Moos D (1995) Fluid flow along potentially active faults in crystalline rock. Geology 23:683:686

Barton C A, Hickman S H, Morin R and Zooback M D (1998) Reservoir-scale fracture permeability in the Dixie Valley, Nevada, Geothermal fields. Society of Petroleum Engineers, SPE Paper no 47371

Bernabe Y (1986) The effective pressure law for permeability in Chelmford granite and Barre granite. Int J Rock Mech Min Sci \& Geomech Abstr 23:267-275

Biot M A (1941) A general theory of three dimensional consolidation. J Appl Phys 12:155-164

Biot M A (1955) Theory of elasticity and consolidation for a porous anisotropic solid. J Appl Phys 26:182-185

Biot M A (1956) General solutions of the equation of elasticity and consolidation for a porous material J Appl Phys 27:240-253

Biot M A and Willis D G (1957) The elastic coefficients of the theory of consolidation. J Appl Mech 24:594-601. 
Boitnott G N (1991) Mechanical and transport properties of joints. PhD, Columbia University, New York

Brace W F, Walsh J B and Frangos W T (1968) Permeability of granite under high pressure. J Geophys Res 73:2225-2236

Brasier F M and Kobelsky B J (1996). Injection of industrial wastes in the United States. In: Apps J and Tsang C-F (eds) Deep Injection of Disposal of Hazardous Industrial Waste. Scientific and engineering aspects. Academic Press, San Diego, California, pp. 18

Brower K R and Morrow N R (1985) Fluid flow in cracks as related to low permeability gas sands Soc Pet Engng J 25:191-201

Brown S R (1987) Fluid flow through rock joints: the effect of surface roughness. J Geophys Res 92:1337-1347

Brown S R and Scholz C H (1986) Closure of rock joints. J Geophys Res 91:4939-4948

Brown D W (1993) Recent flow testing of the HDR reservoir at Fenton Hill, New Mexico. Geothermal Resources Council Bulletin 22:208-214 
Bruno M S, Srinivasa MM, Danyluk PM, Hejl K A and Anduisa J P (1998) Fracture injection of solid oilfield wastes at the West Coyote field in California. In: EUROCK '98, Rock Mechanics in Petroleum Engineering, Society of Petroleum Engineers, SPE Paper no 47371

Byerlee J D (1978) Friction in rocks. J Pure Appl Geophys 73:615-626.

Bäckblom G and Martin C D (1999) Recent experiments in hard rock to study the excavation response: Implication of the performance of a nuclear waste geological repository. Tunneling and Underground Space Technology 14:377-394.

Börgesson L, Pusch R, Fedriksson A, Hökmark H, Karnland O and Sandén S (1992) Final reposrt of the zones disturbed by blasting and stress release. Stripa Project Technical Report 92-08, Swedish Nuclear Fuel and waste management company (SKB), Stockholm.

Carder D S (1945) Seismic investigation in the Boulder Dam area, 1940-1945, and influence of reservoir loading on earthquake activity. Bull Seismol Soc Am 35:175-192.

Carlsson A and Olsson T (1977) Hydraulic properties of the Swedish crystalline rocksHydraulic conductivity and its relationship to depth. Bull. of the Geological Institution of the University of Uppsala, Vol 7, pp 71-84. 
Carlsson and Olsson (1979) Hydraulic conductivity and its stress dependency. In: Proc NEA/IAEA workshop on low-flow, low-permeability measurements in largely impermeable rocks. pp. 249-260.

Carlsson A and Olsson T (1986) Large scale in-situ test on stress and water flow relationships in fractured rock. Swedish State Power Board, Technical Report.

Chen H-Y and Teufel L W (1997) Coupling fluid flow and geomechanics in dualporosity modeling of naturally fractured reservoirs. Society of Petroleum Engineers, SPE Paper no 47371

Clark J B (1949) A hydraulic process for increasing the productivity of wells. Petrl Trans AMIE 186:1-8

Cypser A C and Davis S D (1998) Induced seismicity and the potential for liability under U.S. law. Tectonophysics 289:239-255

Davies J P and Davies J K (1999) Stress-dependent permeability: characterization and modeling. Society of Petroleum Engineers, SPE Paper no 56813.

Detournay, E, Cheng, H-D (1993) Fundamentals of poroelasticity. In: Hudson JA (ed) Comprehensive Rock Engineering. Pergamon Press, Oxford Vol. 1: 395-412 
Doe T W (1999) Evaluating fracture network geometry from hydraulic field data at underground test facilities. In: Faybishenko B (ed) Proc of the International Symposium on Dynamics of fluids in fractured rocks Rocks. Lawrence Berkeley National Laboratory, Berkeley, California. pp 65-70.

DOE (1999) Carbon Sequestration Research and Development, D. Reichle and others (eds.), U.S. Department of Energy Report DOE/SC/FE-1, Washington, DC.

Dusseault M B, Bilak R A, Bruno M S and Rothenburg L (1996) Disposal of granular solid wastes in the western Canadian sedimentary basic by slurry injection. In: Apps J and Tsang C-F (eds) Deep Injection of Disposal of Hazardous Industrial Waste. Scientific and engineering aspects. Academic Press, San Diego, California, pp. 725-742

Dusseault M (1997) Geomechanics in oil and gas exploration and drilling. Keynote lecture at the $1^{\text {st }}$ Asian Rock Mechanics Symposium. Final program, pp. 100-114.

Dyke C (1995) How sensitive is natural fracture permeability at depth to variation of in effective stress? In: Myer L, Cook N G W, Goodman D and Tsang C-F (eds) Fracture and Jointed Rock Masses, pp. 81-88

Eliasson T M, Sundquist U and Wallroth T (1990) Ciculation experiments in 1989 at Fjällbacka HDR test site. Report Fj-10, Department of Geology, Chalmers University. 
Elliot G M, Brown E T, Boodt P I and Hudson J A (1985) Hydromechanical behavior of joint in the Carmenelis granite, SW England. In: Stephansson O (ed) Proc Int Symp Fundamentals of rock Joints, Börkliden, Sweden, pp. 249-258

Emsley S, Olsson O, Stenberg L, Alheid H-J and Fall S (1997) ZEDEX — a study of damage and disturbance from tunnel excavation by blasting and tunnel boring. Swedish Nuclear Fuel and Waste Management Co (SKB) Technical Report 97-30, Stockholm Engelder T and Scholz C H (1981) Fluid flow along very smooth joints at effective pressures up to 200 megapascals. In Carter N L (ed) Mechanical Behavior of Crustal Rocks, Monograph 24, Am Geophys Union (AGU), Washington D.C., pp. 147-152.

Esaki T, Du S, Mitani Y, Ikusada K and Jing L (1999) Development of a shear-flow test apparatus and determination of coupled properties for a single rock joint. Int J Rock Mech Min Sci \& Geomech Abstr 36:641-650

Evans D (1966) The Denver area earthquakes and the Rocky Mountain Arsenal Disposal Well. Mtn Geology 3:23-36

Evans K F, Kohl T, Hopkirk R J and Rybach L (1992) Modeling of energy production from Hot Dry Rock Systems. Project Report Eidgenössische Technische Hochschule (ETH). 
Evans KF, Cornet FH, Hashida T, Hayashi, K, Ito T, Matsuki, K, Wallroth T (1999) Stress and rock mechanics isssues of relevance to HDR/WDR engineered geothermal systems: review of developments during the past 15 years. Geothermics 28:455-474

Fairhurst C (1964) Measurements of in situ rock stresses with particular reference to hydraulic fracturing. Rock Mech Eng Geol 2:129-147.

Ferrill D, Winterle J, Wittmeyer G, Sims D, Colton S and Armstrong A (1999) Stressed rock strains groundwater at Yucca Mountain, Nevada. GSA Today, Geological Society of America, May 1999.

Gale J (1975) A numerical, field and laboratory study of flow in rock with deformable fractures PhD University of California, Berkeley.

Galloway D, Jones D R and Ingebritsen S E (eds) (1999) Land Subsidence in The United States. US Geological Survey Circulation 182, Reston, Virginia.

Gambolati G P and Freeze A (1973) Mathematical simulation of the subsidence of Venice. I. Theory. Water Resourc Res 9:721-733.

Gambolati G, Ricceri G, Bertoni W, Brighenti G and Vuillermin E (1991). Mathematical simulation of the subsidence of Ravenna. Water Resourc Res 27:2899-2918 
Gangi A F (1978) Variation of whole and fractured porous rock permeability with confining pressure. Int J Rock Mech Min Sci \& Geomech Abstr 15:249-257

Gaucher E, Cornet F H and Bernard P (1998) Induced seismicity analysis for structure identification and stress field determination. In: EUROCK '98, Rock Mechanics in Petroleum Engineering, Society of Petroleum Engineers, SPE Paper no 47324, pp. 545554

Geertsma J (1957) The effect of fluid-pressure decline on volumetric changes of porous rocks. Trans AIME 210:331-339

Geertsma J (1966) Problems of rock mechanics in petroleum production engineering. In: Proc $1^{\text {st }}$ International Congress on Rock Mechanics, Lisbon, Vol I, pp 585-594

Geertsma J and Deklerk F (1969) A rapid method of predicting width and extent of hydraulically induced fractures. J Pet Tech 21:1571-1581

Geertsma J (1973) Land subsidence above compacting oil and gas reservoirs. J Pet Tech, 25:734-744

Gerrard C M (1982) Elastic models of rock masses having one, two and three sets of joints. Int J Rock Mech Min Sci \& Geomech Abstr 19:15-23 
Ghaboussi J and Wison E L (1973) Flow of compressible fluids in porous elastic media. Int J Numer Methods Eng 5:419-442.

Goodman R E (1970) Deformability of joints. In: Symposium on Determination of the InSitu Modulus of Deformation of Rock, ASTM, S T P 477, pp. 174-196.

Goodman R E (1974) The mechanical properties of joints. In: Proc $3^{\text {rd }}$ International Congress of the International Society of Rock Mechanics, Vol I, pp 127-140.

Goodman R E (1976) Methods of geological engineering in discontinuous rock, West Publishing, New York.

Gupta H K (1992) Reservoir induced earthquakes. Elsevier, New York.

Gutierrez M, Barton N and Makurat A (1995) Compaction and subsidence in North Sea hydrocarbon fields. In: Yoshinaka R and Kikuchi K (eds) Proc of the international workshop on rock foundation, Tokyo, Japan, A.A. Balkema, Rotherdam. pp. 57-64.

Gutierrez M and Lewis R W (1998) The role of geomechanics in reservoir simulation. In: EUROCK '98, Rock Mechanics in Petroleum Engineering, Society of Petroleum Engineers, SPE Paper no 47392, pp. 439-448. 
Habib P (1987) The Malpasset dam failure. Engineering Geology 24:331-338

Haimson B C and Fairhurst C (1967) Initiation and extension of hydraulic fractures in rocks. Soc Pet Engng J Sept:310-318

Hainey B W, Keck R G, Smith M B, Lynch K W and Barth J W (1997) On-site disposal of oilfield waste solids in Wilmington Field, Long Beach Unite, CA. Society of Petroleum Engineers, SPE Paper no 47371.

Hakami E (1995) Aperture distribution of rock fractures. PhD, Royal Institute of Technology, Sweden.

Harpalani S and Schraufnagel R A (1990) Shrinkage of coal matrix with release of gas and its impact on permeability of coal. Fuel 199:551-556.

Harpalani S and Chen G (1995) Estimation of changes in fracture porosity of coal with gas emission. Fuel 74:1491-1498.

Hayashi K and Abé H (1989) Evaluation of hydraulic properties of the artificial subsurface system in Higashihachimantai geothermal model field. Journal of the Geothermal Research Society of Japan 11:203-215 
Healy J H, Rubey W W, Griggs D T and Raleigh C B (1968) The Denver earthquakes. Science 161:1301-1310

Heiland J (2003) Laboratory testing of coupled Hydro-mechanical processes during rock deformation. This volume.

Henkel H (2002) The Bjökö geothermal energy project. Norges geologiske undersökelse Bulletin 439: 45-50

Hsieh P A and Bredehoeft J D (1981) A reservoir analysis of the Denver earthquakes: a case of induced seismicity. . J Geophys Res 86:903-925

Hubbert M K and Willis D G (1957) Mechanics of hydraulic fracturing. J Pet Tech 9:153-168

Ito T and Zoback M D (2000) Fracture permeability and in situ stress to $7 \mathrm{~km}$ depth in the KTB scientific drillhole. Geophys Res Let 27:1045-1048

Iwai K (1976) Fundamental studies of fluid flow through a single fracture. PhD, University of California, Berkeley. 
Iwano M (1995) Hydromechanical characteristics of a single rock joint. $\mathrm{PhD}$, Massachusetts Institute of Technology.

Jacob C E (1940) On the flow of ground water in a elastic artesian aquifer. Trans Am Geophys Union 22:783-787

Jacob C E (1950) Flow of groundwater. In: Engineering hydraulics, John Wiley, New York, pp. 321-386.

Jing L and Hudson J A (2002) Numerical methods in rock mechanics. Int J Rock Mech Min Sci 39: 409-427

Jones F O jr (1975) A laboratory study of the effects of confining pressure on fracture flow and storage capacity in carbonate rocks. J Pet Tech, 27:21-27

Jones F O and Ovens W W (1980) A laboratory study of low permeability gas sands. J Pet Eng 32:1631-1640

Jung R (1989) Hydraulic in situ investigation of an artificial fracture in the Falkenberg granite. Int J Rock Mech Min Sci \& Geomech Abstr 26:301-308 
Jupe A J, Green A S P and Wallroth (1992) Induced microseismicity and reservoir growth at the Fjällbacka Hot Dry Rock Project, Sweden. Int J Rock Mech Min Sci \& Geomech Abstr 29:343-354

Kilmer N H, Morrow N R and Pitman J K (1987) Pressure sensitivity of low permeability sandstones. J Pet Sci and Eng 1:65:81.

Koutsabeloulis N C and Hope S A (1998) “Couped" stress/fluid/thermal multi-phase reservoir simulation studies incorporating rock mechanics. In: EUROCK '98, Rock Mechanics in Petroleum Engineering, Society of Petroleum Engineers, SPE Paper no 47392, pp. 449-454.

Kranz R L, Frankel A D, Engelder T and Scholz C H (1979) The permeability of whole and jointed barre granite. Int J Rock Mech Min Sci \& Geomech Abstr 16:225-234.

Lee C-I and Chang K-M (1995) Analysis of permeability change and groundwater flow around underground oil storage cavern in Korea. In: Fujii T (ed) Proc $8^{\text {th }}$ Int Congr Rock Mechanics, pp. 779-782.

Lewis R W and Sukirman Y (1993) Finite element modeling of three-phase flow in deforming saturated oil reservoirs. In J Num Anal Meth Geomech 17:577-598 
Lewis R W and Schrefler BA (1998) The Finite Element Method in the Static and Dynamic Deformation and Consolidation of Porous Media, 2nd Edition. John Wiley \& Sons

Lewis RW, Pao WKS and Makurat A (2003) Fully coupled modeling of seabed subsidence and reservoir compaction of North Sea oil fields. This issue.

Liu J and Elsworth D (1997) Three-dimensional effects of hydraulic conductivity enhancement and desaturation around mined panels. Int. J. Rock Mech. Min. Sci $34: 1139-1152$

Liu J, Elsworth D and Brady B H (1999) Linking stress-dependent effective porosity and hydraulic conductivity fields to RMR. Int. J. Rock Mech. Min. Sci 36:581-596

Londe P and Sabarly F (1966) La distribution des perméabilitésdans la fondation des barrages voûtes en fonction du champ de contrainte. In: Proc $1^{\text {st }}$ International Congress on Rock Mechanics, Lisbon, Vol II, pp. 517-522.

Londe P (1987) The Malpasset dam failure. Engineering Geology 24:295-329.

Louis C and Maini Y (1970) Determination of in situ hydraulic parameters in jointed rock. In: Proc $2^{\text {nd }}$ International Congress on Rock Mechanics, Vol I, pp 235-245. 
Louis C, Dessenne J_L and Feuga B (1977) Interaction between water flow phenomena and the mechanical behavior of soil or rock masses. In: Gudehus G (ed) Finite elements in geomechanics. John Wiley \& Sons, pp. 479-511.

Makurat A, Barton N and Rad N S (1990) Joint conductivity variation due to normal and shear deformation. In: Barton N and Stephansson (eds) Rock Joints pp 535-540

Martin C D, Davison C C and Kozak E T (1990) Characterizing normal stiffness and hydraulic conductivity of a major shear zone in granite. In: Barton N and Stephansson (eds) Rock Joints pp 549-556

Meinzer O E (1928) Compressibility and elasticity of artesian aquifers. Econ Geol 23:263-291

Myer L R (1991) Hydromechanical and seismic properties of fractures. In: Proc $7^{\text {th }}$ Int Congr Rock Mechanics, pp. 397-404

Nagelhout A C G and Roest J P A (1997) Investigating fault slip in a model of underground gas storage facility. Int J Rock Mech Min Sci \& Geomech Abstr 34:Paper no 212 
Nakatsuka K (1999) Field characterization for HDR/WDR: a review. Geothermics 28:519-531

National Research Council (1996) Rock Fractures and Fluid Flow, National Academy Press, Washington D.C.

Neuzil C E and Tracy J V(1981) Flow through fractures. Water Resourc Res 17:191-199

Neuzil C E (1986) Ground water flow in low-permeability environments. Water Resourc Res 22:1163-1195

Neuzil C E (2003) Hydromechanical coupling in geologic processes. This volume.

Nicholson C and Wesson R L (1992) Triggered earthquakes and deep well activities. Pure Appl Geophys 139:561-578

Nolte K G (1991) Fracturing-pressure analysis for nonideal behavior. J Pet Tech Feb 1991: $210-218$

Noorishad J (1971) Finite element analysis of rock mass behavior under coupled action of body forces, fluid flow, and external loads. PhD, University of California, Berkeley. 
Noorishad J, Ayatollahi M S, Witherspoon P A (1982) A finite element method for coupled stress and fluid flow analysis of fractured rocks. Int. J. Rock Mech. Min. Sci. \& Geomech. Abstr. 19:185-193

Noorishad J, Tsang C-F and Witherspoon P A (1992) Theoretical and field studies of coupled hydromechanical behavior of fracture rocks-1 Development and verification of a numerical simulator. Int. J. Rock Mech. Min. Sci. \& Geomech. Abstr. 29:401-409

Nur A and Byerlee J D (1971) An exact effective stress law for elastic deformation of rock with fluids. J Geophys Res 76:6414-6419

Oda M (1982) Fabric tensor for discontinuous geological materials. Soils and Foundations 22:96-108

Olsson O (1992) Site characterization and validation-final report: Stripa Project, Swedish Nuclear Fuel and Waste Management Co, TR 92-22, Stockholm

Olsson R and Barton N (2001) An improved model for hydromechanical coupling during shearing of rock joints. Int J Rock Mech Min Sci 38:317-329

Osoiro J G, Chen H-Y, Teufel L W and Schaffer S (1998) A two-domain, 3D, fully coupled fluid flow/geomechanical simulation model for reservoirs with stress sensitive 
mechanical and fluid-flow properties. In: EUROCK '98, Rock Mechanics in Petroleum Engineering, Society of Petroleum Engineers, SPE Paper no 47397, pp. 455-464.

Patton F D (1966) Multiple modes of shear failure in rock. In: Proc $1^{\text {st }}$ International Congress on Rock Mechanics, Lisbon, Vol I, pp 509-513

Perkins T K and Kern L R (1961) Widths of hydraulic fractures. J Pet Tech Sept 1961:937-949.

Pine RJ, Batchelor, AS (1984) Downward migration of shearing in jointed rock during hydraulic injections. Int J Rock Mech Min Sci 21:249-263

Pratt W E and Johnson D W (1926) Local subsidence of the Goose Creek oil field. J Geology 34:577-590

Pratt H R, Swolfs H S, Brace W F, Black A D and Handin J W (1977) Elastic and transport properties of an in situ jointed granite. Int. J. Rock Mech. Min. Sci. \& Geomech. Abstr. 14: 35-45

Pyrak-Nolte L, Myer L J, Cook N G W and Witherspoon P A (1987) Hydraulic and mechanical properties of natural fractures in low permeability rock. In: Proc $6^{\text {th }}$ Congress of the International Society of Rock Mechanics, Montreal, Vol 1, pp. 225-231. 
Raven K G and Gale J E (1985) Water flow in a natural rock fracture as a function of stress and sample size. Int. J. Rock Mech. Min. Sci. \& Geomech. Abstr. 22: 251-261

Read R S and Chandler N A (1999) Excavation damage and stability studies at the URL. In: Amadei B, Kranz R L, Scott G A and Smeallie P H (eds) Proc $37^{\text {th }}$ US Rock Mechanics Symposium Vol 2, pp. 861-868

Reed A C, Mathews J L, Bruno M S and Olmstead SE (2001) Chevron safely disposes one million barrels of NORM in Louisiana through slurry fracture injection. Society of Petroleum Engineers, SPE Paper no 47371

Rejeb A (1996) Mathematical simulation of coupled THM processes of Fanay-Augéres fiedl test by distinct element and discrete finite element methods. In Stephansson O, Jing L and Tsang C-F (eds) Coupled thermo-hydro-mechanical processes of fractured media, Elsevier, pp. 341-369

Rhén I, Gustafson G, Stanford R and Wikberg P (1997) Äspö HRL - Geoscientific Evaluation 1997-5: Models based on site characterization 1986-1995. Swedish Nuclear Fuel and Waste Management Company (SKB), Technical Report 97-06.

Rice J R and Cleary M P (1976) Some basic stress diffusion solutions of fluid saturation elastic porous media with compressible constituents. Rev Geophys Space Phys 14:227241 
Richards H G, Parker R H, Green A S P, Jones R H, Nicholls J D M, Nichol D A C, Randall M M, Stewart R C and Willis-Richards J (1994) The performance characteristics of the experimental Hot Dry Rock Geothermal Reservoir at Rosemanowes, Cornwall (1985-1988). Geothermics 23:73-109

Rutqvist J (1995a) Coupled stress-flow properties of rock joints from hydraulic field testing. PhD, Royal Institute of Technology, Sweden

Rutqvist J (1995b) Determination of hydraulic normal stiffness of fractures in hard rock from hydraulic well testing. Int J Rock Mech Min Sci \& Geomech Abstr 32: 513-523

Rutqvist (1995c) Hydraulic pulse testing of single fractures in porous and deformable rocks. Q J Eng Geol 29:181-192

Rutqvist J, Tsang C-F, Ekman D and Stephansson O (1997) Evaluation of in situ hydromechanical properties of rock fractures at Laxemar in Sweden. In Proc $1^{\text {st }}$ Asian Rock Mechanics Symposium ARMS 97, Seoul, Korea, pp. 619-624

Rutqvist J, Tsang C-F and Stephansson O (1998) Determination of fracture storativity in hard rocks using high pressure testing. Water Resourc Res 34:2551-2560 
Rutqvist J and Tsang C-F (2002) Analysis of thermal-hydrologic-mechanical behavior near an emplacement drift at Yucca Mountain. Journal of Contaminant Hydrology (in press).

Rutqvist J, Wu Y-S, Tsang C-F and Bodvarsson G (2002) A modeling approach for analysis of coupled multiphase fluid flow, heat transfer, and deformation in fractured porous rock. Int. J. Rock Mech. Min. Sci. 39:429-442

Saeb S and Amadei B (1992) Modeling Rock Joints Under Shear and Normal Loading. Int. J. Rock Mech. Min. Sci. 29:267-278

Sandhu R S and Wilson E L (1969) Finite-element analysis of seepage in elastic media. J Engineering Mech Div ASCE 95:641-652

Sasaki S (1998) Characteristics of microseismic events induced during hydraulic fracturing experiments at the Hijiori hot dry rock geothermal energy site, Yamagata, Japan. Tectonophysics 289:171-188.

Scheidegger A E (1974) The Physics of Flow Through Porous Media. University of Toronto Press, Toronto.

Scholz C H (1990) The Mechanics of Earthquakes and Faulting. Cambridge University Press, New York. 
Schmidt J H, Friar W L, Bill M L and Cooper G D (1999) Large scale injection of North Slope Drilling Cuttings. Society of Petroleum Engineers, SPE Paper no 52738

Schmitt DR, Zoback MD (1989) Poroelastic effects in the determination of the maximum horizontal principal stress in hydraulic fracturing tests - a proposed breakdown equation employing a modified effective stress relation for tensile failure. Int. J. Rock Mech. Min. Sci. \& Geomech. Abstr. 26: 499-506

Schrauf T W and Evans D D (1986) Laboratory studies of gas flow through a single natual fracture. Water Resourc Res 22:1038-1050

Segall P (1989) Earthquakes triggered by fluid extraction. Geology 17:942-946.

Segall P and Fitzgerald S D (1998) A note on induced stress changes in hydrocarbon and geothermal reservoirs, Tectonophysics 289:17-128.

Simpson D W, Leith W S, Scholz C H (1988) Two types of reservoir-induced seismicity. Bull Seismol Sco Am 78:2025-2040

Snow D T (1965) A Parallel Plate Model of Fractured Permeable Media. PhD, University of California, Berkeley. 
Snow D T (1968) Rock fracture spacings, openings, and porosities. J Soil Mech Found Div ASCE 73-91

Snow D T (1969) Anisotropic permeability of fractured media. . Water Resourc Res $5: 1273-1289$

Souley M, Homand F, Pepa S and Hoxha D. (2001) Damage-induced permeability changes in granite: a case example at the URL in Canada. Int. J. Rock Mech. Min. Sci. 38: 297-310.

Stephansson O, Jing L and Tsang C F (eds) (1996) Coupled Thermo-hydro-mechanical processes of fractured media. Elsevier, Amsterdam.

Stietel A, Millard A, Treille E, Vuillod E, Thoravel A and Ababou R (1996) Continuum representation of coupled hydromechanical processes of fractured media. In Stephansson O, Jing L and Tsang C-F (eds) Coupled thermo-hydro-mechanical processes of fractured media, Elsevier, pp. 135-164.

Stowel J F W, Laubch S E and Olson J E (2001) Effect of modern state of stress on flowcontrolling fractures: a misleading paradigm in need of revision. In: Elsworth D, Tinucci J P and Heasley K A (eds) Proc $38^{\text {th }}$ US Rock Mechanics Symposium Vol I, pp. 691-697. 
Sugihara K, Matsu H and Sato T (1999) In situ experiments on rock stress conditions and excavation disturbance in JNC's geoscientific research program in Japan. In: Saeb S and Francke C (eds) Rock Mechanics of Nuclear Waste Repositories, Veil, Colorado. pp. 159-183.

Sutton S, Soley R, Jeffery C, Chackrabarty C and McLeod R (1996) Cross hole hydraulic testing between deep boreholes 2 and 4 at Sellafield. European Commission, Nuclear Sciences Technology Topical Report EUR 16967, Brussels.

Swan G (1983) Determination of stiffness and other properties from roughness measurements. Rock Mech and Rock Engng 16:19-38

Takahashi M, Koide H and Xue Z-Q (1995) Correlation between permeability and deformational characteristics on the Shirahama sandstone and Inada granite. In: Fujii T (ed) Proc Eighth Int Congr Rock Mechanics, pp. 417-420.

Talbot C J and Sirat M (2001) Stress control of hydraulic conductivity in fracturedsaturated Swedish bedrock. Engineering Geology 61:145-153

Terzaghi, K. (1923) Die Berechnung der Durchlässigkeitziffer des Tonesaus dem Verlauf der hydrodynamischen Spannungserscheinungen, Akad. Der Wissenschaften in Wien, Sitzungsberichte, Mathematisch-naturwissenschafttliche Klasse, Part IIa 142(3/4), 125138. 
Teufel L W and Farrel H E (1995) Interrelationship between in situ stress, natural fractures, and reservoir permeability anisotropy-A case study of the Ekofisk Field, North Sea. In: Myer L, Cook N G W, Goodman D and Tsang C-F (eds) Fracture and Jointed Rock Masses, pp. 573-578.

Theis C V (1935) The relationship between the lowering of the piesometric surface and rate and duration of the discharge of a well using groundwater storage. Trans Am Geophys Union 2:519-524.

Thury M and Bossart P (1999) The Mont Terri rock laboratory, a new international research project in a Mesozoic shale formation, in Switzerland. Engineering Geology $52: 347-359$

Tsang Y W and Witherspoon P A (1981) Hydromechanical behavior of a deformable rock fracture subject to normal stress. J Geophys Res 86:9287-9298

Tsang Y W (1984) The effect of tortuosity on fluid flow through a single fracture. Water Resourc Res 20:1209-1215

Tsang C-F (1987) Introduction to coupled processes. In Tsang C-F (ed) Coupled Processes Associated with Nuclear Waste Repositories, pp. 1-6. 
Van-Golf Racht T D (1982) Fundamentals of fractured reservoir engineering. Elsevier, Amsterdam.

Verruijt (1969) Elastic storage of aquifers. In: DeWiest R J M (ed) Flow through Porous Media, Academic Press, New York, pp. 331-376.

Walsh J B (1965) The effect of cracks on the compressibility of rock. J Geophys Res 70:381-389.

Walsh J B (1981) Effects of pore pressure and confining pressure on fracture permeability. Int. J. Rock Mech. Min. Sci. \& Geomech. Abstr. 18:429-435

Wang J S Y and Elsworth D (1999) Permeability changes induced by excavation in fractured tuff. In: Amadei B, Kranz R L, Scott G A and Smeallie P H (eds) Proc $37^{\text {th }}$ US Rock Mechanics Symposium Vol 2, pp. 751-758.

Wang H.F (2000) Theory of linear poroeasticity, Princeton Univ. Press, 287p.

Wang J, Cook P, Trautz R, Flexser S. Hu, Q, Salve R, Hudson D, Conrad M, Tsang Y, Williams K, Soll and W, Turin J (2001) In-Situ Field Testing of Processes ANL-NBS-HS000005 REV01. Las Vegas, Nevada: Bechtel SAIC Company. 
Wang J-A and H D Park (2002) Fluid permeability of sedimentary rocks in a complete stress-strain process. Engineering Geology 63:291-300.

Wei Z-Q and Hudson J A (1988) Permeability of jointed rock masses. In: Romana M (ed) Rock Mechanics and Power Plants, Balkema Rotterdam, pp. 613-626.

Withers R J, Perkins T K and Keck R G (1996) A field demonstration of hydraulic fracturing for solid waste disposal. In: Apps J and Tsang C-F (eds) Deep Injection of Disposal of Hazardous Industrial Waste. Scientific and engineering aspects. Academic Press, San Diego, California pp. 705-724

Witherspoon P A, Amick C H, Gale J E and Iwai K (1979) Observations of a potential size effect in experimental determination of the hydraulic properties of fractures. Water Resourc Res 15:1142-1146.

Witherspoon P A, Wang J S Y, Iwai K and Gale J E (1980) Validity of cubic law for fluid flow in a deformable rock fracture. Water Resourc Res 16:1016-1024

Witherspoon P A (2000) The Stripa project. Int. J. Rock Mech. Min. Sci. 37:385-396

Wladis D, Jönsson P and Wallroth T (1997) Regional characterization of hydraulic properties or rock using well test data. Swedish Nuclear Fuel and Waste Management Company (SKB), Technical Report 97-29. 
Xiao HT, Xu HY (2000) In situ permeability measurements to establish the influence of slice mining on floor rock. Int. J. Rock Mech. Min. Sci. 37:855-860

Yoshinaka R, Yoshida J, Arai H and Arisaka S (1993) Scale effects on shear and deformability of rock joints. In: Pinto da Chunha A (ed) Scale Effects in Rock Masses 93, pp. $143-149$

Zhao J and Brown E T (1992) Hydro-thermo-mechanical properties of joints in the Crarnmenellis granite. Q J Eng Geol 25:279-290

Zienkiewics O C and Pande G N (1977) Time-dependent multilaminate model of rocks-a numerical study of deformation and failure of rock masses. Num Anal Meth Geomech 1:219-247.

Zoback M D and Byerlee J D (1975) The effect of microcrack dilatancy on the permeability of westerly granite. J Geophys Res 80:752-755. 


\section{LIST OF FIGURE CAPTIONS}

Figure 1. Schematic overview of a fractured geological medium composed of an intact porous rock matrix and macrofractures

Figure 2. Hydromechanical couplings in geogical media. (i) and (ii) are direct couplings through pore volume interactions while (iii) and (iv) are indirect couplings through changes in material properties

Figure 3. Literature data of permeability versus effective confining pressure for intact rock of Pierre shale (average from Neuzil 1986), Westerly granite (Brace et al. 1968) and MWX tight sand gas (Kilmer et al. 1987)

Figure 4. Typical mechanical and hydromechanical fracture responses under normal closure ( $a$ and $b$ ) and shear (c and d). Effects of sample size is indicated with the laboratory sample response (dashed lines) compared to with in situ fracture response (1 $\mathrm{m}^{2}$ size)

Figure 5. Fracture hydraulic conductivity versus normal stress for various sample sizes of artificial and natural tension fractures (from Witherspoon et at. 1977)

Figure 6. (a) Vertical cross section and conceptual model of the US Geological Survey's fractured rock research site near Mirror Lake, New Hampshire. Four clusters of highly 
permeable fractures labeled A, B, C and D occur in the less permeable fractured rocks. Borehole packers are shown in black (from National Research Council 1996)

Figure 7. Inflow features in the drift system at the storage site for low- and intermediatelevel nuclear waste, Forsmark, Sweden (From Carlsson and Olsson, 1977). The depicted inflow features can be described as: (1) outflow in open channels in a major fracture, (2) evenly distributed flow along subhorizontal tension joints, (3) flow focused to the termination of a steep fracture at a subhorizontal fracture, (4) enhanced flow at fracture intersections and (5) diffuse flow through unfractured rock.

Figure 8. Conceptual three-dimensional view of a highly water conductive minor shear fault located at Äspö Hard Rock Laboratory, Sweden (Hakami 1995). The aperture variation is simplified in the model into open areas (white; $b>0.1 \mathrm{~mm}$ ) and contact areas (shaded; $\mathrm{b} \leq 0.1 \mathrm{~mm}$ ). The elliptical shaped contact areas reflects the anisotropy in correlation distances obtained from aperture measurements with $\mathrm{d} 1$ and $\mathrm{d} 2$ typically 8 and $16 \mathrm{~cm}$, respectively

Figure 9. In situ determination of normal stress versus transmissivity relationship using a combination of pulse, constant head and hydraulic jacking tests. (a) Schematic representation of pressure and flow versus time, (b) the radius of influence in each test and (c) results of hydraulic jacking test (Rutqvist et al. 1997) 
Figure 10. In situ measurements of hydraulic aperture and apparent physical aperture during hydraulic jacking tests into an artificial fracture in granite at a depth of about 250 meters (data from data of Jung 1989)

Figure 11. Permeability measured in short interval well tests in fractured crystalline rocks at Gideå, Sweden (data points from Wladis et al. 1997). Effects of shear dislocation and mineral precipitation/dissolution processes obscure the dependency of permeability on depth (stress). The permeability values on the left hand side represents intact rock granite, or flow feature 5 in Figure 7, while the permeability values on the right hand side represents highly conductive fractures, possible flow feature 1 in Figure 7

Figure 12. Schematics of land subsidence at the Wilmington Oil Field in California. (a) Horizontal strain causing horizontal faulting and (b) vertical and horizontal displacement (from Segall 1989)

Figure 13. Stress change in reservoir caused by a decrease in reservoir pore pressure. The minimum horizontal stress was determined by hydraulic fracturing (from Segall 1998)

Figure 14. Schematics of faulting associated with underground extraction of oil- and gas (Segall 1989)

Figure 15. Injection flow rate, injection pressure and seismic activities during fluid injection at Hijiori Hot dry rock site in Japan. (a) A one day high pressure hydraulic 
fracturing operation and (b) a one month low pressure circulation experiment (modified from Sasaki 1998)

Figure 16. Spatial distribution of the micro-seismic events at Hijiori Hot dry rock site in Japan. (a) Events during a one day high pressure hydraulic fracturing operation. (b) Events during a one month low pressure circulation experiment (modified form Sasaki (1998))

Figure 17. Londe's hypothesis for the 1959 failure at the Malpassat Dam, France. The figure shows how an underground hydraulic barrier was created under load of the dam due to extremely stress-sensitive permeability (modified from Londe (1987)) 
Figure 18. Excavation induced pressure changes in rocks during tunnel boring at the Grimsel Test Site in Switzerland. The pressure is measured in a 10 meter long packed-off section of a borehole located about 3 meters from the wall of the tunnel (see sketch of tunnel and monitoring section below the pressure curve)

Figure 19. Schematic of the Excavation Disturbed Zone (EDZ) around a drift in fractured rock

Figure 20. Pre- to post excavation permeability tests conducted in fractured unsaturated tuff at Yucca Mountain, Nevada. (a) Geometry of tunnel and test boreholes and (b) Ratio of Pre- and post excavation permeability plotted against pre-excavation permeability (from Wang et al 2002)

Figure 21. Flow and pressure response during a constant pressure injection into a subhorizontal fracture located at 70 meters depth. The injection pressure is $1.2 \mathrm{MPa}$ and the vertical stress normal to the fracture is estimated to $2.0 \mathrm{MPa}$. Note that the injection rate increases during the first 60 minutes despite a reducing pressure gradient. This can be explained by an increased permeability as a result of increased fluid pressure within the fracture (data from Alm 1999)

Figure 22. Development of the HDR reservoir concepts in the last 25 years. (a) Penny shaped fracture, (b) shear on natural joints and (c) graben concept. After Baria et al. (1999a) 
Figure 23. Schematic picture showing possible permeability changes at shallow and deep locations in fractured bedrock. The solid lines represent the depth- (or stress)permeability function for intact rock, clean tension joint and highly conductive and locked open fractures 


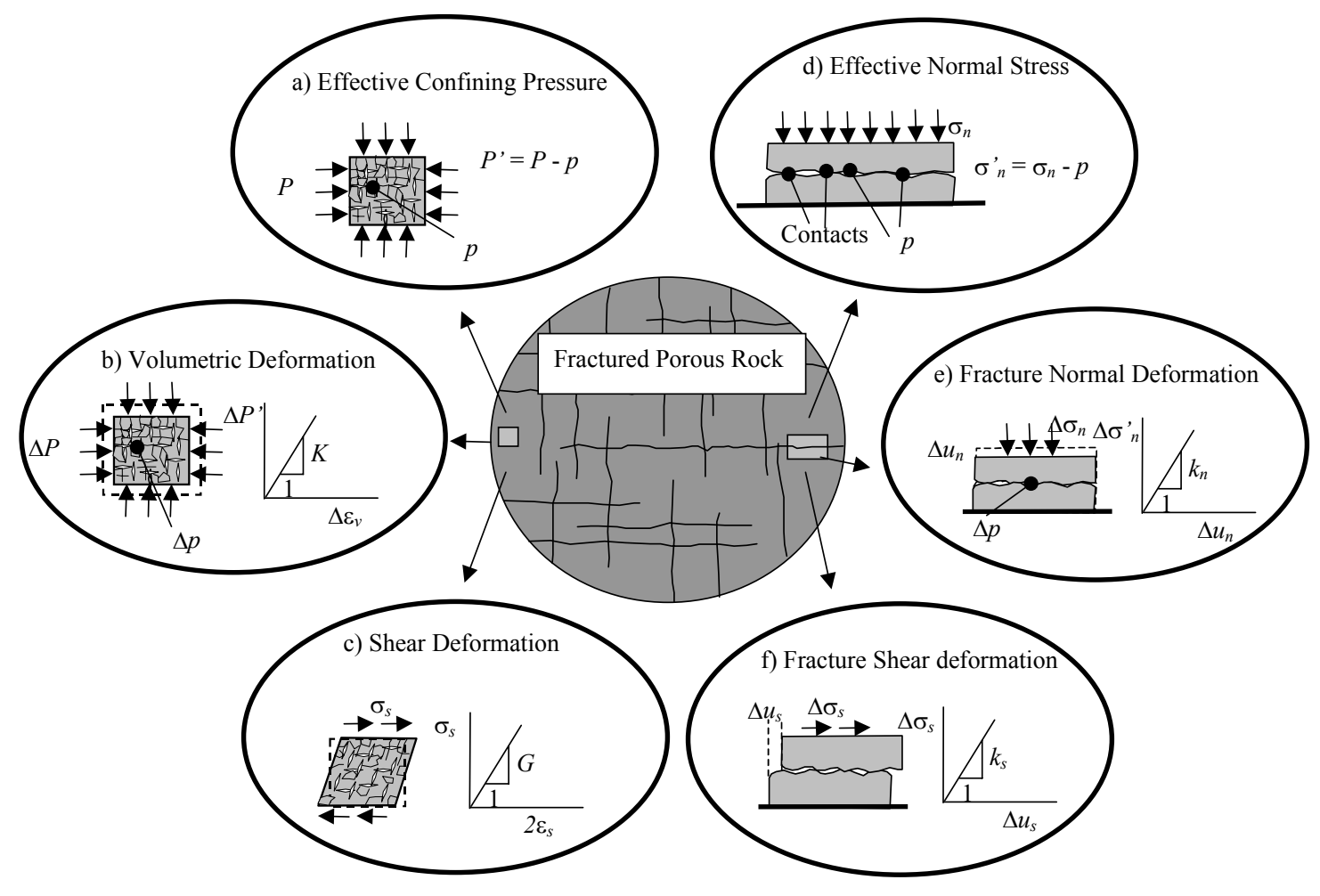

Figure 1. Schematic overview of a fractured geological medium composed of an intact porous rock matrix and macrofractures 


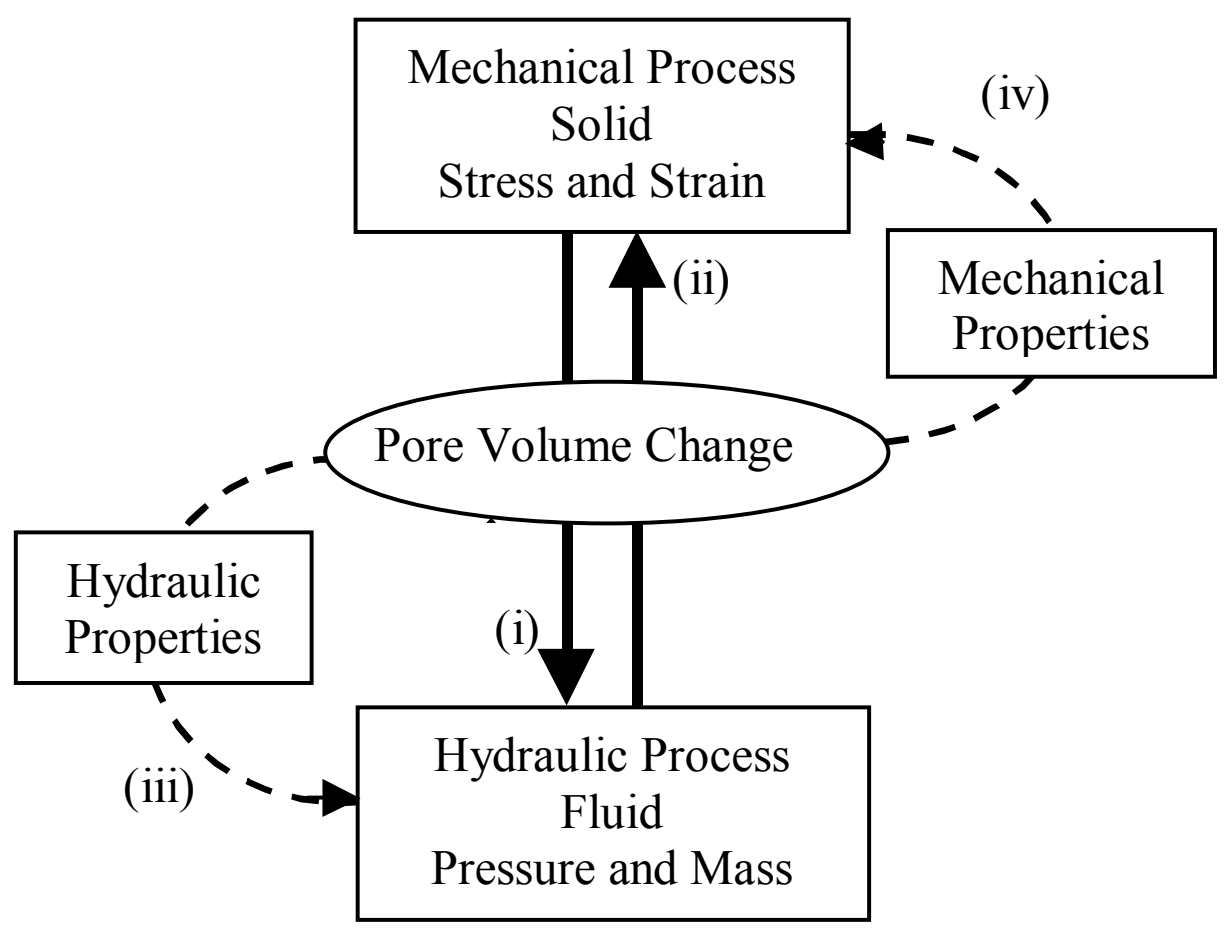

Figure 2. Hydromechanical couplings in geogical media. (i) and (ii) are direct couplings through pore volume interactions while (iii) and (iv) are indirect couplings through changes in material properties 


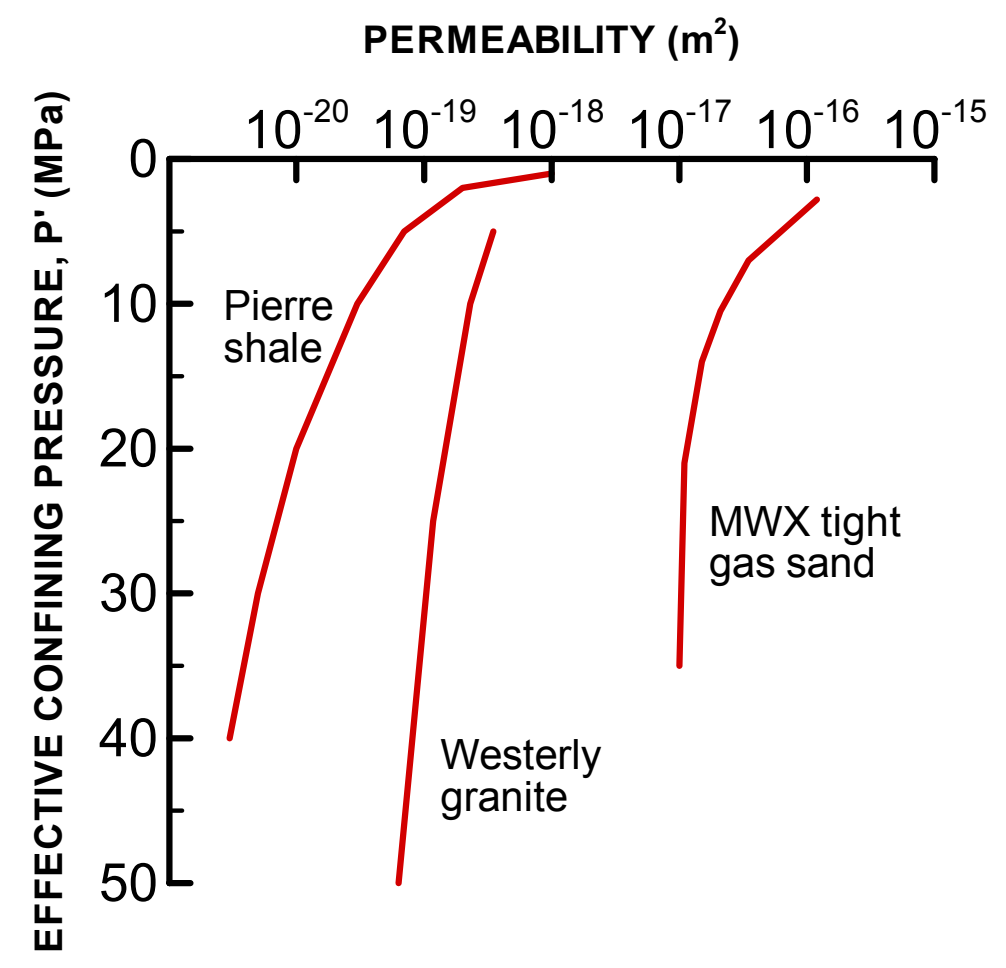

Figure 3. Literature data of permeability versus effective confining pressure for intact rock of Pierre shale (average from Neuzil 1986), Westerly granite (Brace et al. 1968) and MWX tight sand gas (Kilmer et al. 1987) 


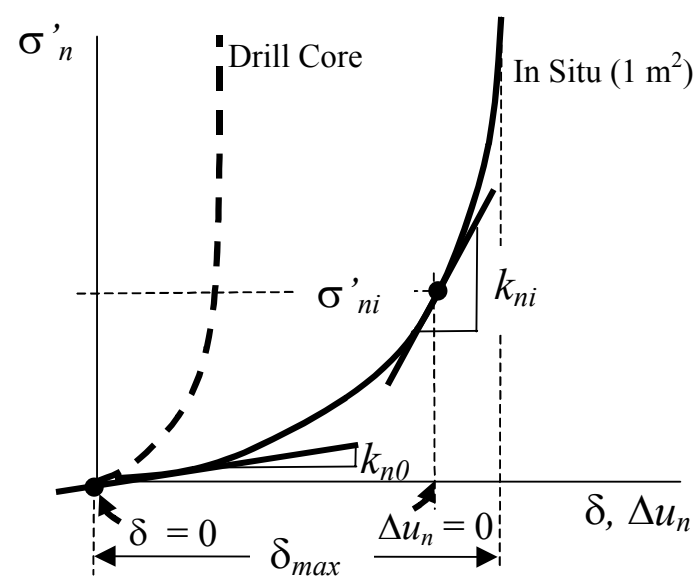

(a)

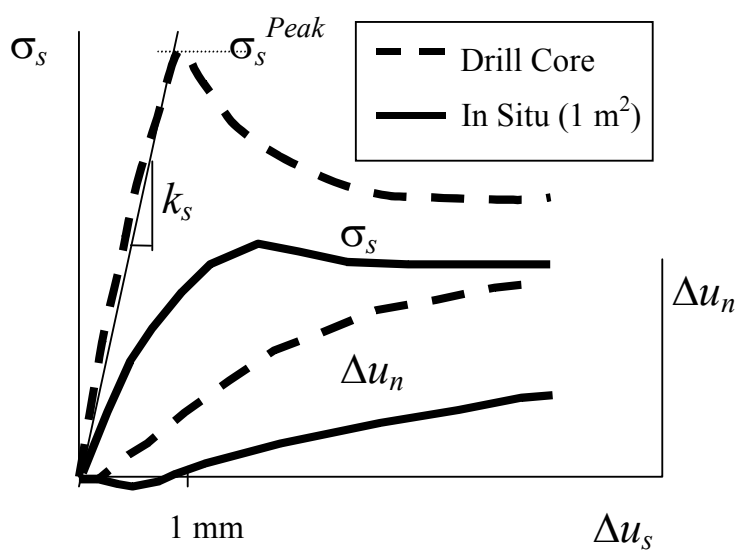

(c)

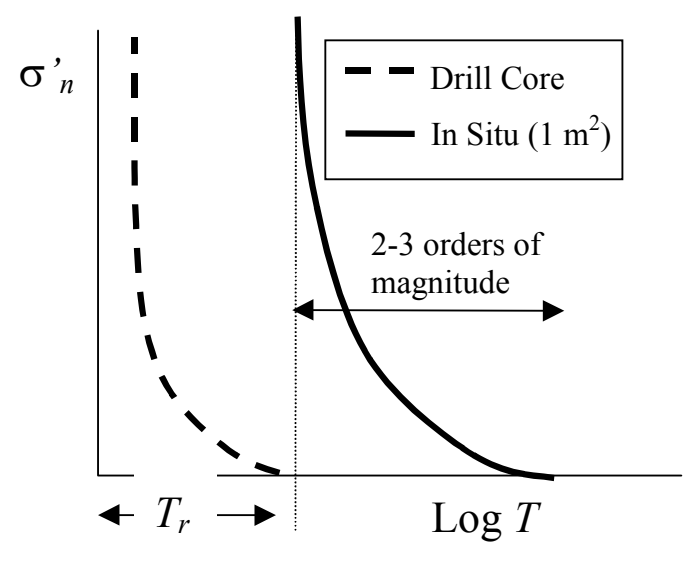

(b)

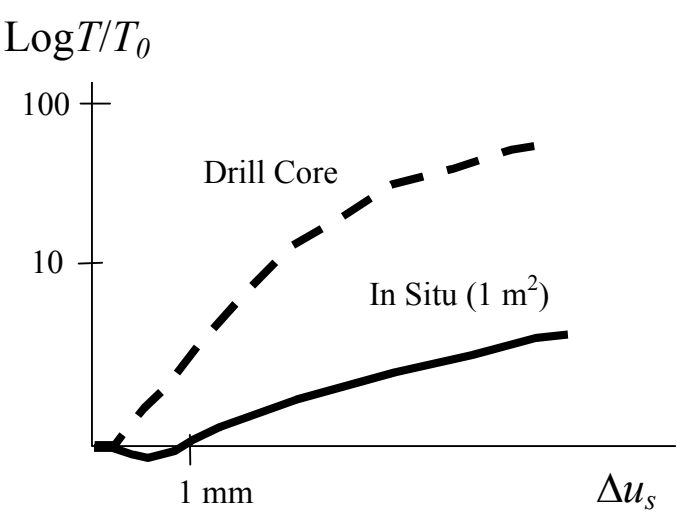

(d)

Figure 4. Typical mechanical and hydromechanical fracture responses under normal closure ( $a$ and $b$ ) and shear ( $c$ and d). Effects of sample size is indicated with the laboratory sample response (dashed lines) compared to with in situ fracture response (1 $\mathrm{m}^{2}$ size) 


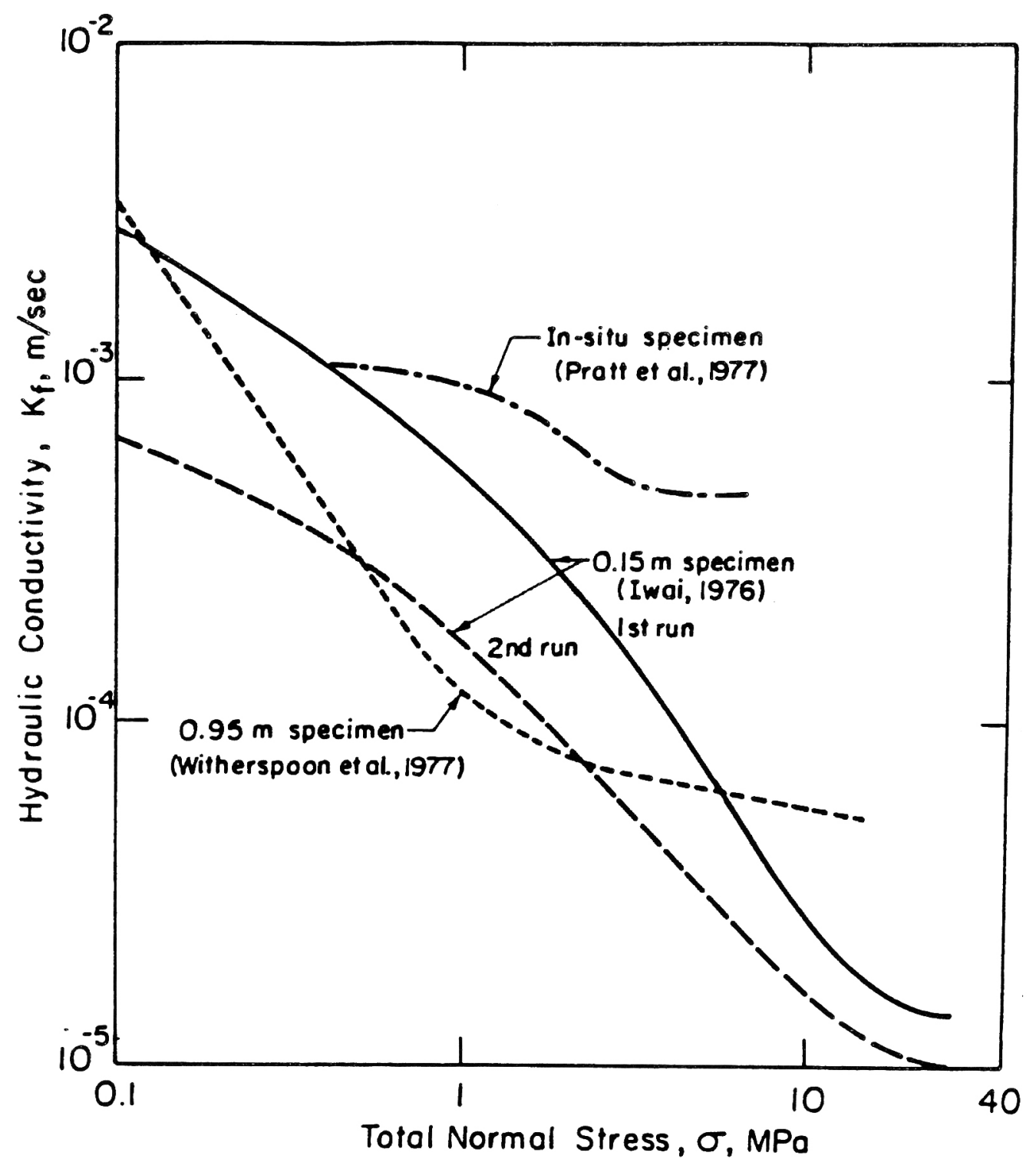

Figure 5. Fracture hydraulic conductivity versus normal stress for various sample sizes of artificial and natural tension fractures (from Witherspoon et at., 1977) 


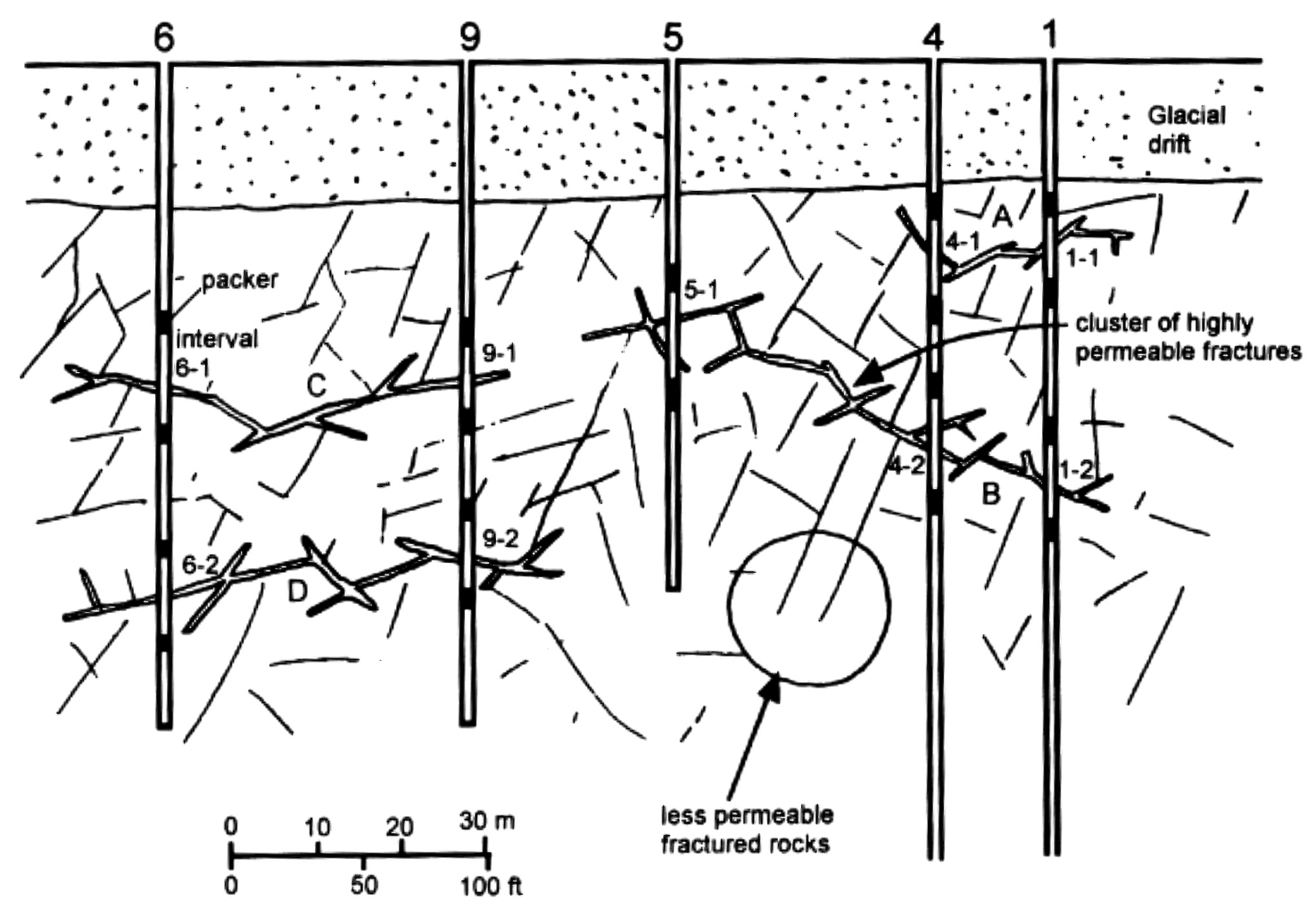

Figure 6. (a) Vertical cross section and conceptual model of the US Geological Survey's fractured rock research site near Mirror Lake, New Hampshire. Four clusters of highly permeable fractures labeled A, B, C and D occur in the less permeable fractured rocks. Borehole packers are shown in black (from National Research Council, 1996) 


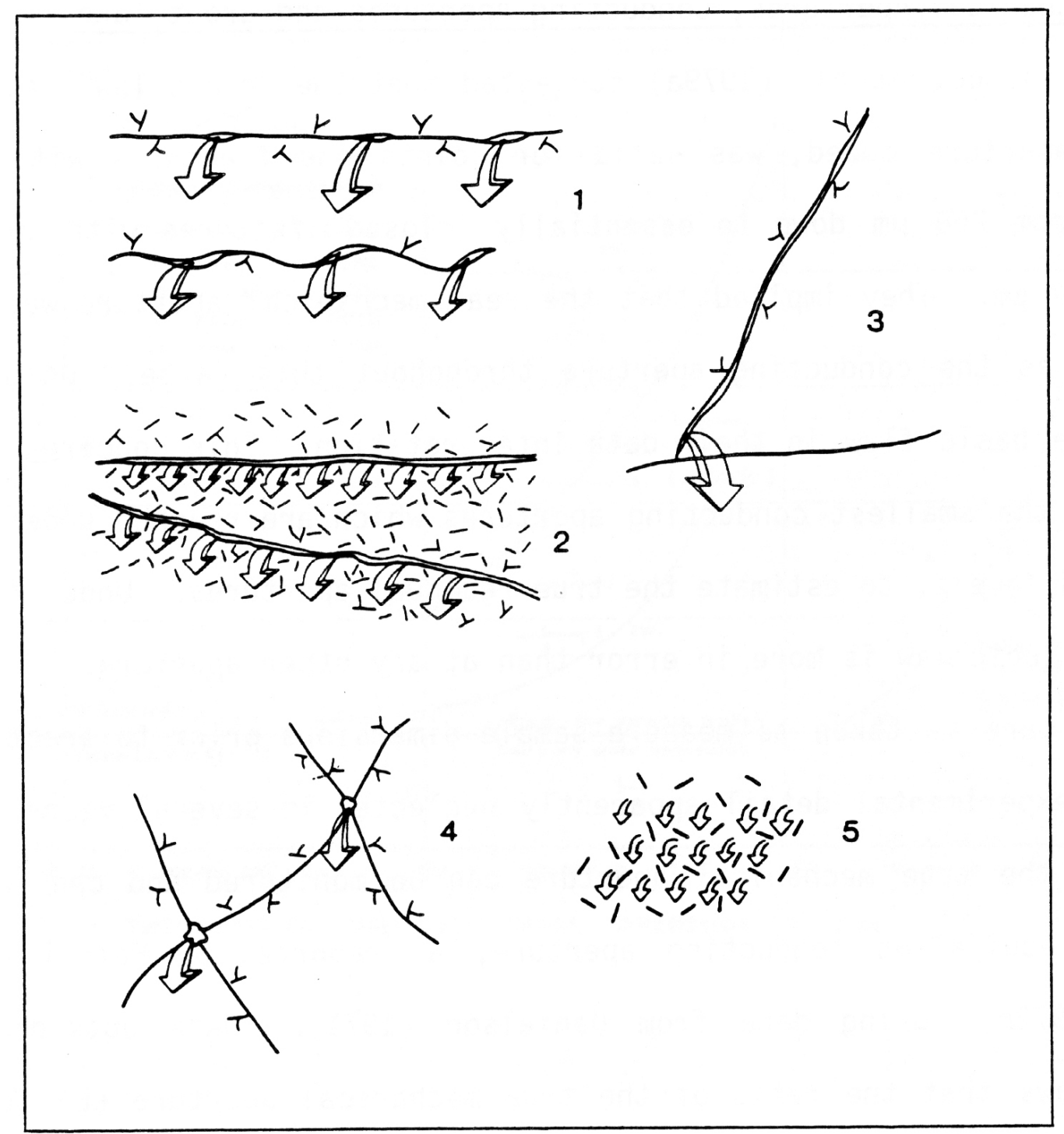

Figure 7. Inflow features in the drift system at the storage site for low- and intermediatelevel nuclear waste, Forsmark, Sweden (From Carlsson and Olsson, 1977). The depicted inflow features can be described as: (1) outflow in open channels in a major fracture, (2) evenly distributed flow along subhorizontal tension joints, (3) flow focused to the termination of a steep fracture at a subhorizontal fracture, (4) enhanced flow at fracture intersections and (5) diffuse flow through unfractured rock. 


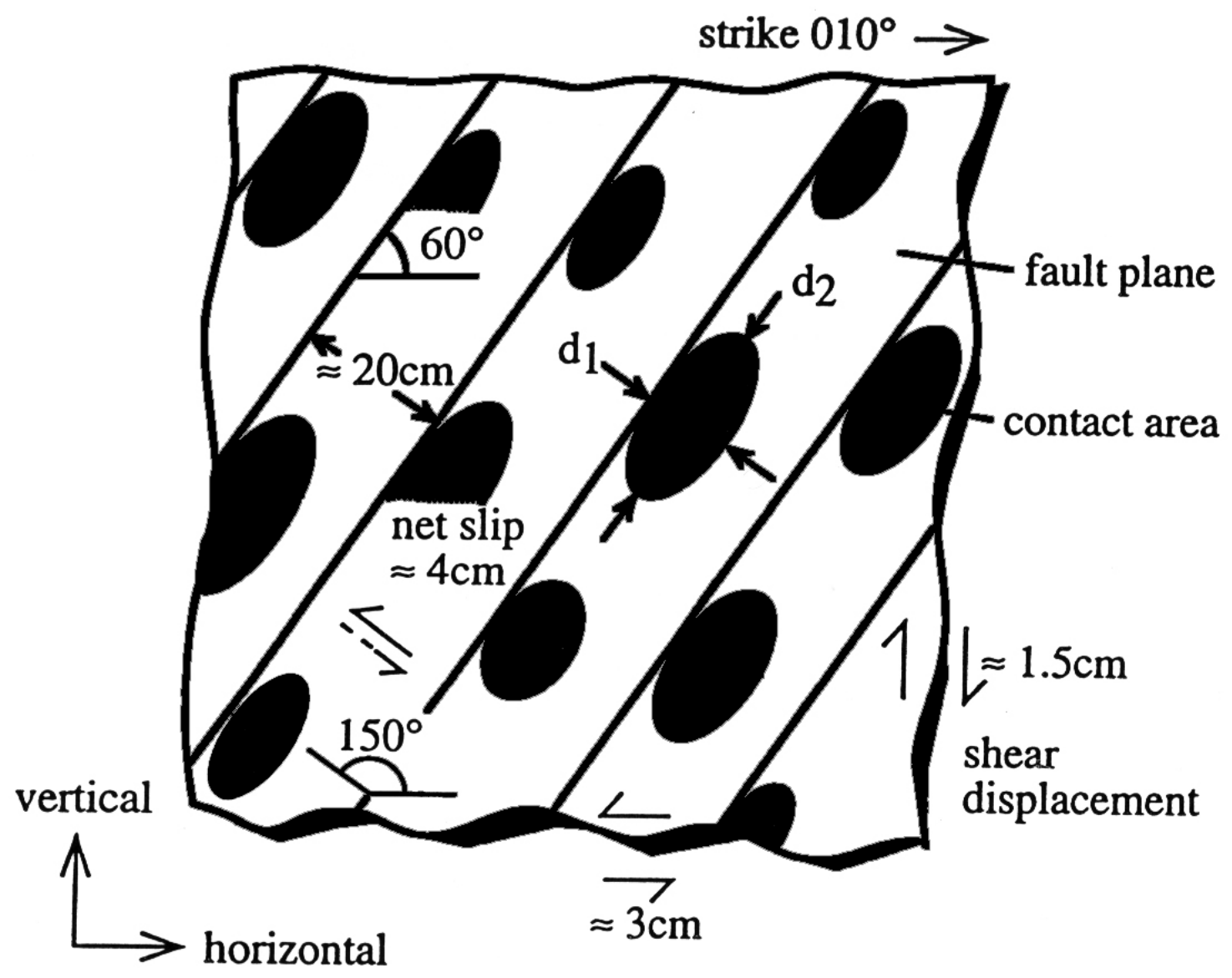

Figure 8. Conceptual three-dimensional view of a highly water conductive minor shear fault located at Äspö Hard Rock Laboratory, Sweden (Hakami, 1995). The aperture variation is simplified in the model into open areas (white; $b>0.1 \mathrm{~mm}$ ) and contact areas (shaded; $b \leq 0.1 \mathrm{~mm}$ ). The elliptical shaped contact areas reflects the anisotropy in correlation distances obtained from aperture measurements with $\mathrm{d} 1$ and $\mathrm{d} 2$ typically 8 and $16 \mathrm{~cm}$, respectively 


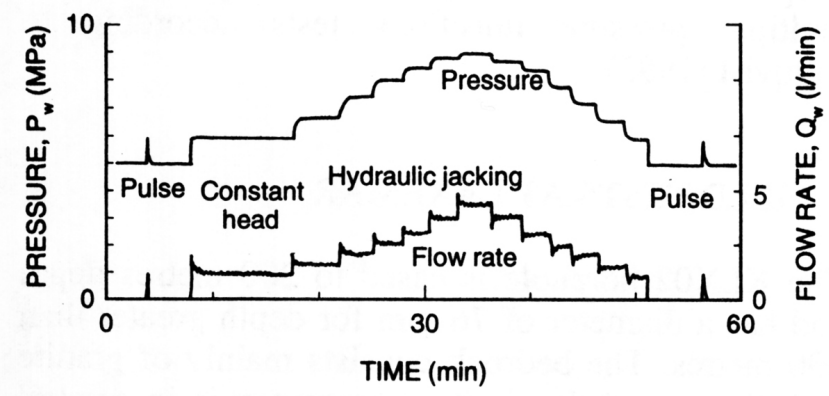

a)
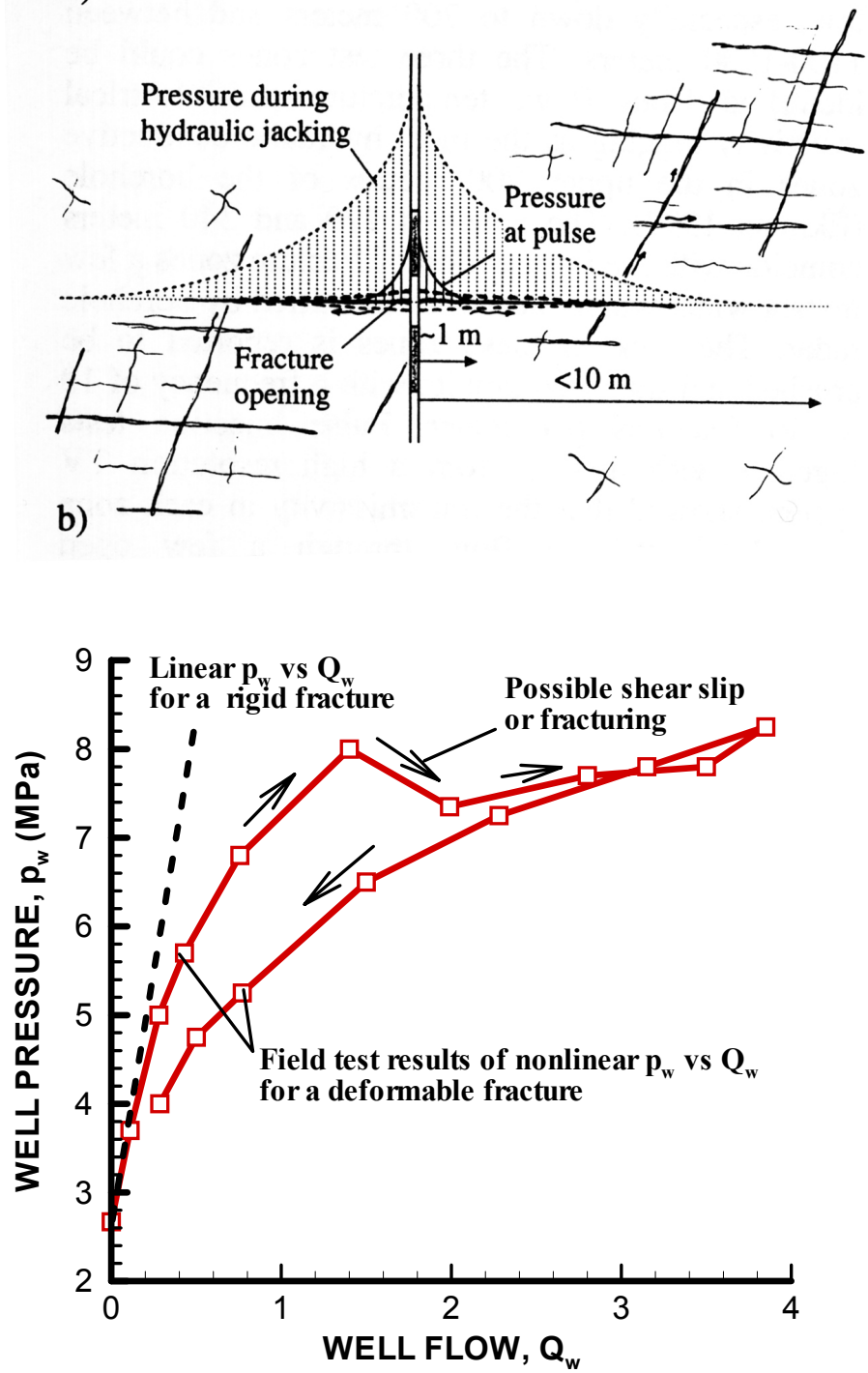

(c)

Figure 9. In situ determination of normal stress versus transmissivity relationship using a combination of pulse, constant head and hydraulic jacking tests. (a) Schematic representation of pressure and flow versus time, (b) the radius of influence in each test and (c) results of hydraulic jacking test (Rutqvist et al. 1997) 


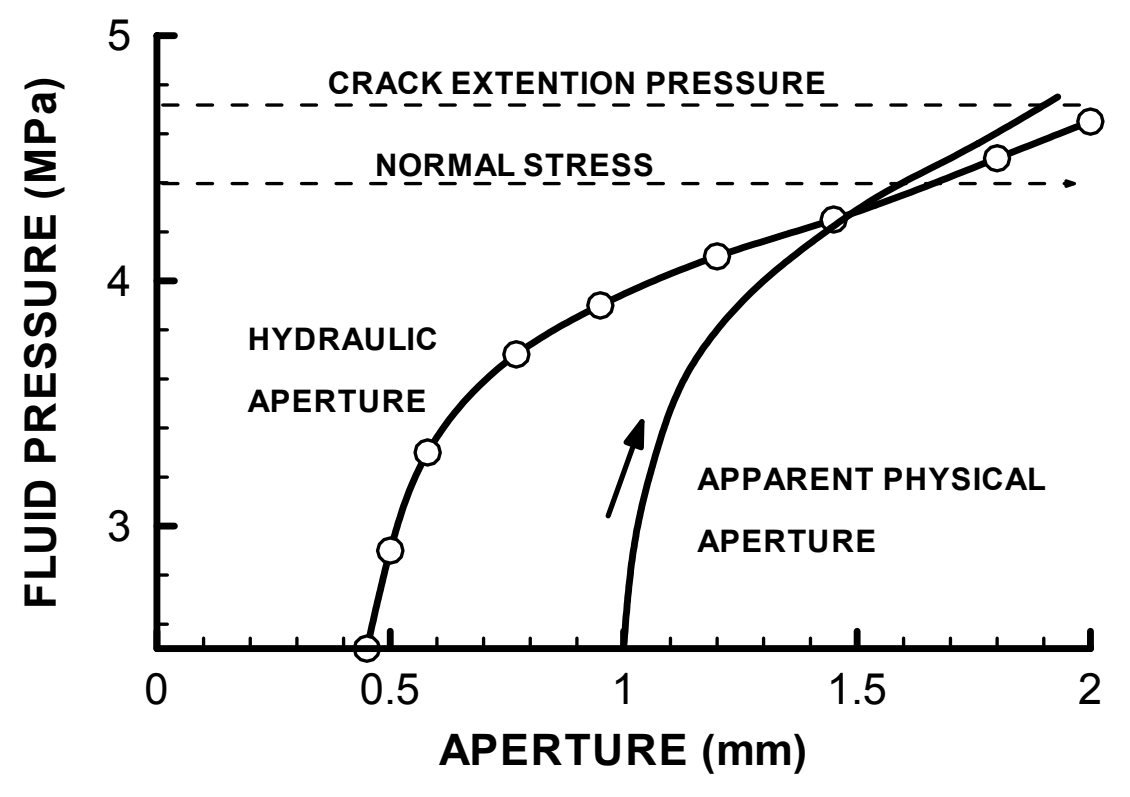

Figure 10. In situ measurements of hydraulic aperture and apparent physical aperture during hydraulic jacking tests into an artificial fracture in granite at a depth of about 250 meters (data from data of Jung 1989) 


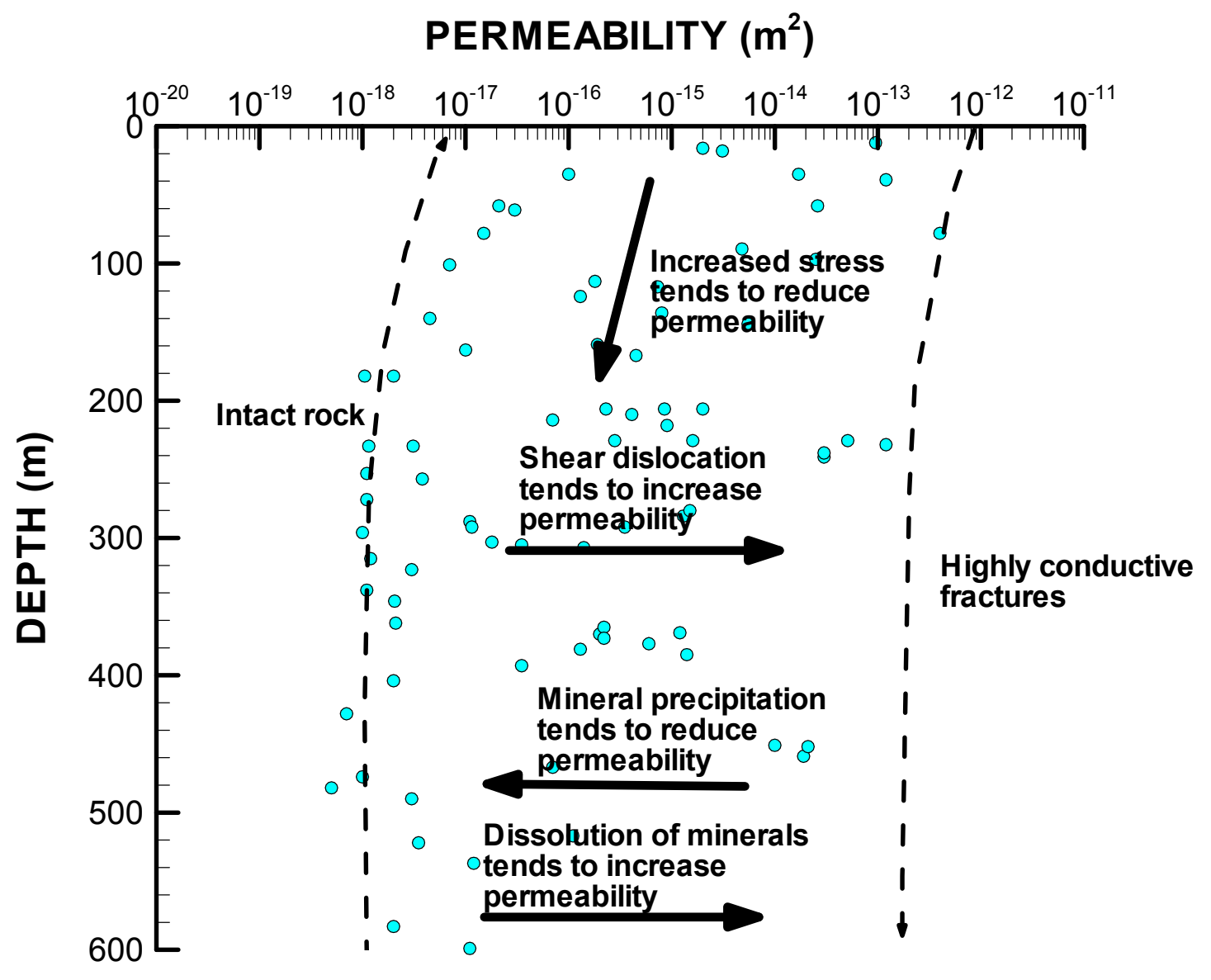

Figure 11. Permeability measured in short interval well tests in fractured crystalline rocks at Gideå, Sweden (data points from Wladis et al. 1997). Effects of shear dislocation and mineral precipitation/dissolution processes obscure the dependency of permeability on depth (stress). The permeability values on the left hand side represents intact rock granite, or flow feature 5 in Figure 7, while the permeability values on the right hand side represents highly conductive fractures, possible flow feature 1 in Figure 7 


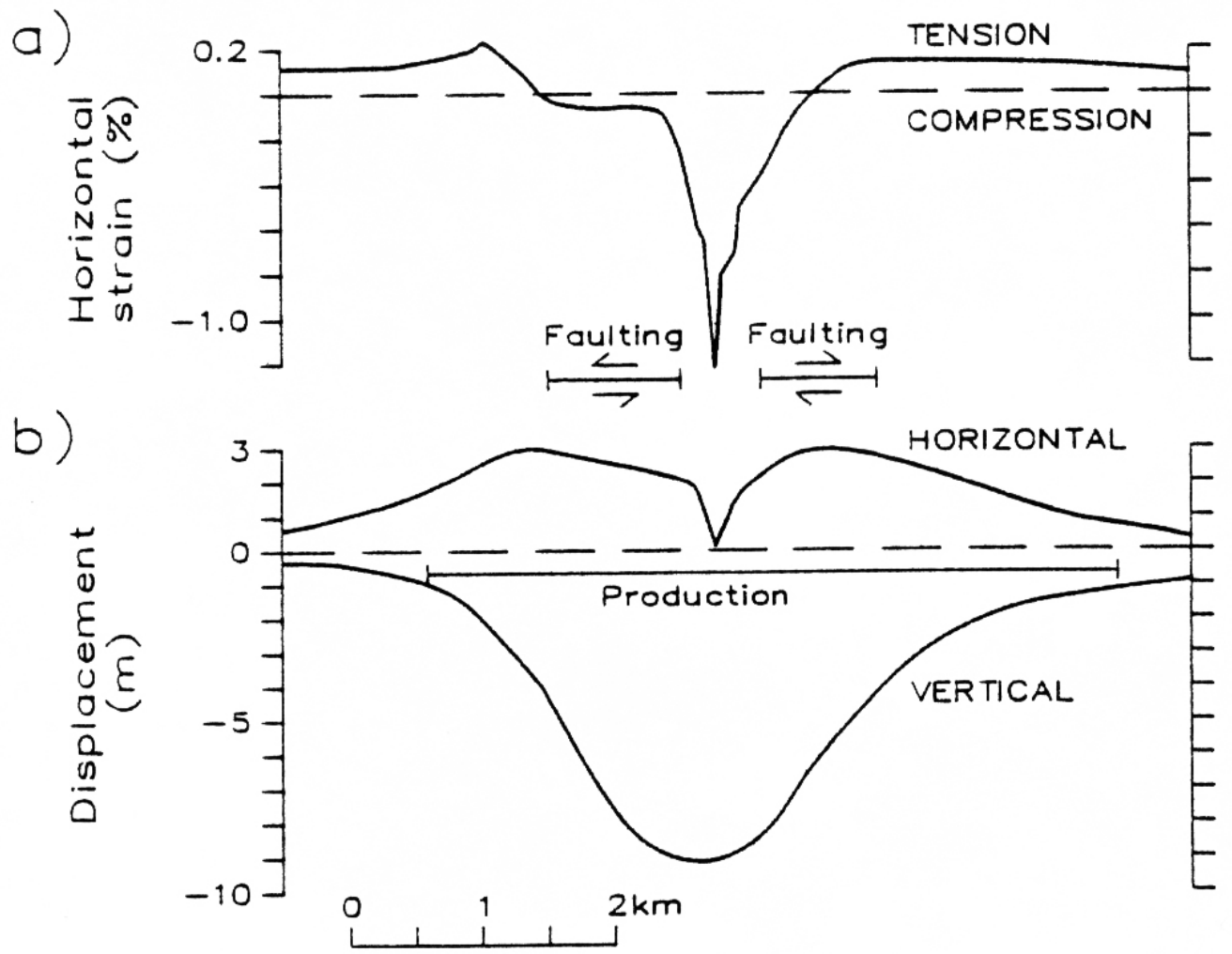

Figure 12. Schematics of land subsidence at the Wilmington Oil Field in California. (a) Horizontal strain causing horizontal faulting and (b) vertical and horizontal displacement (from Segall 1989) 


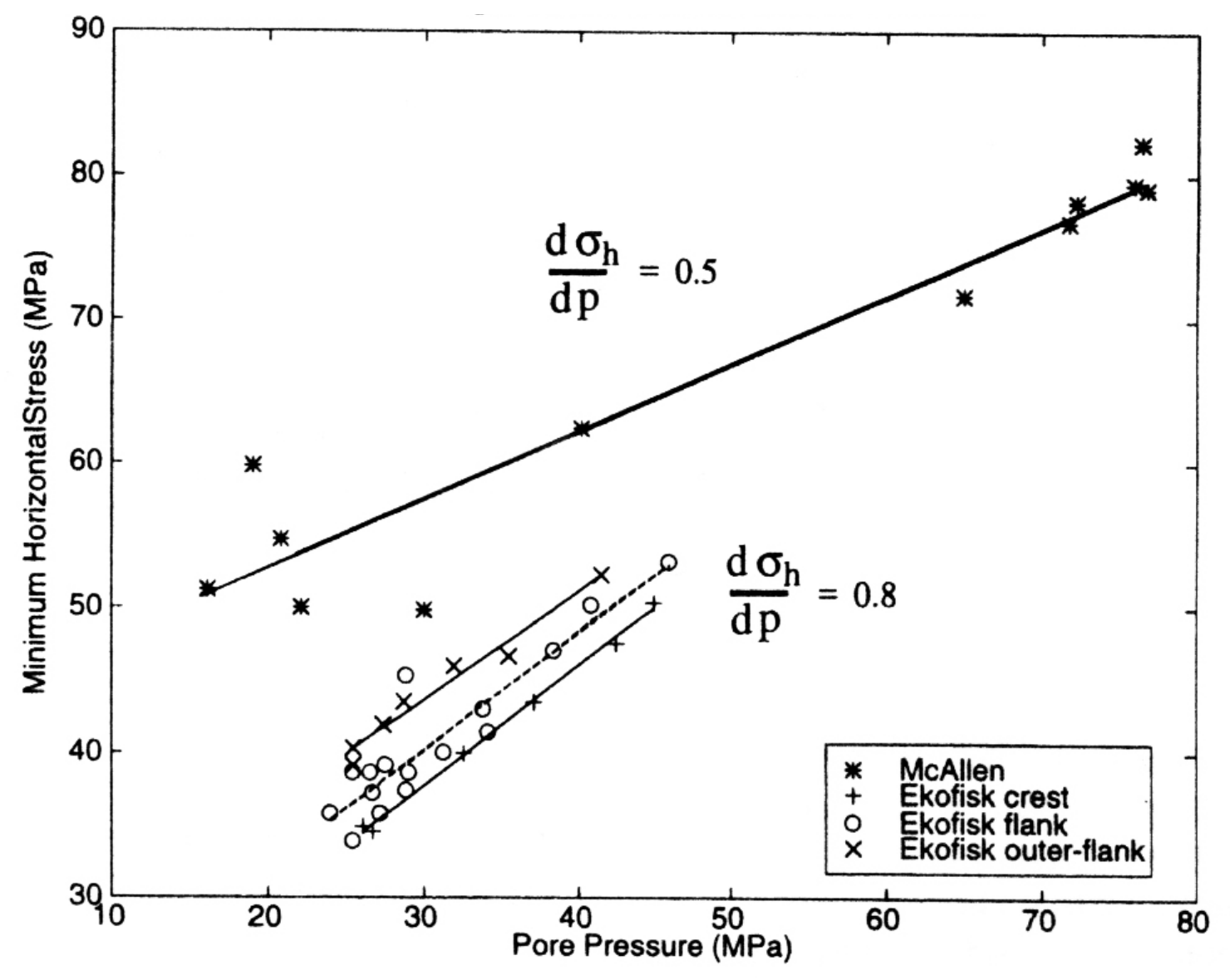

Figure 13. Stress change in reservoir caused by a decrease in reservoir pore pressure. The minimum horizontal stress was determined by hydraulic fracturing (from Segall 1998) 


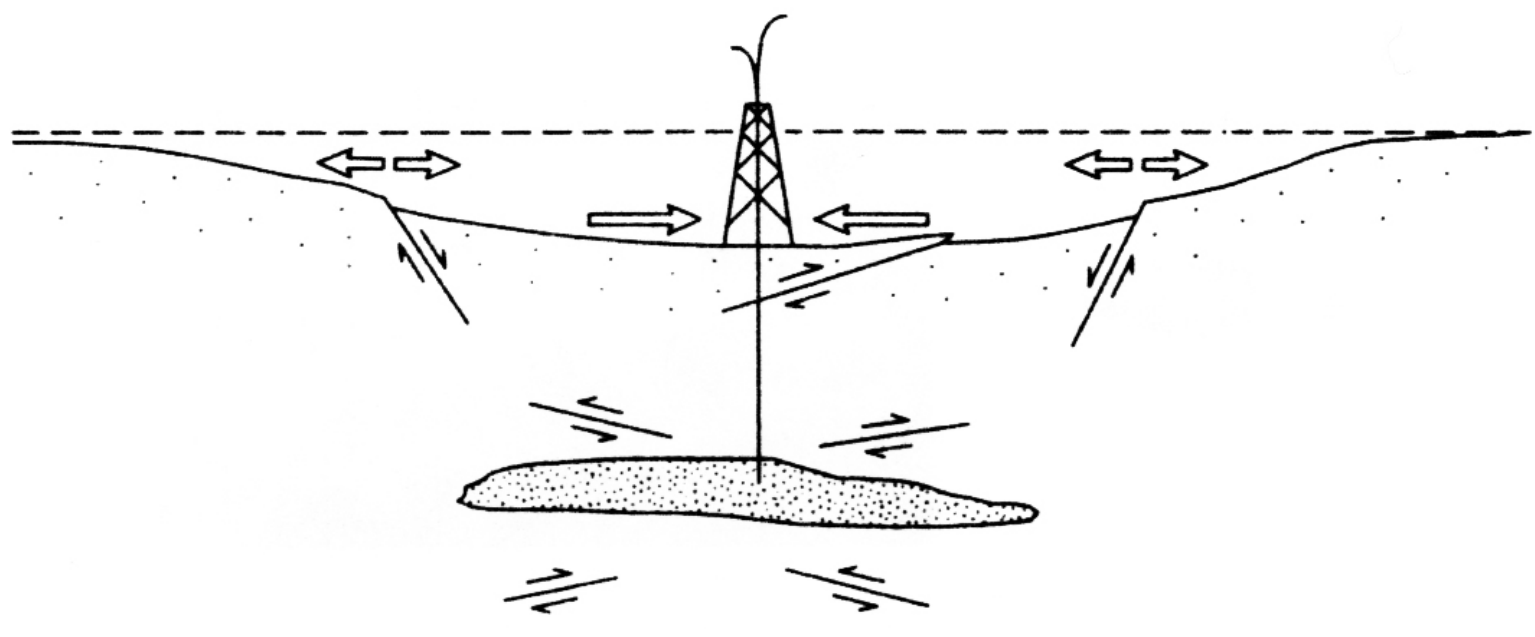

Figure 14. Schematics of faulting associated with underground extraction of oil- and gas (Segall 1989) 

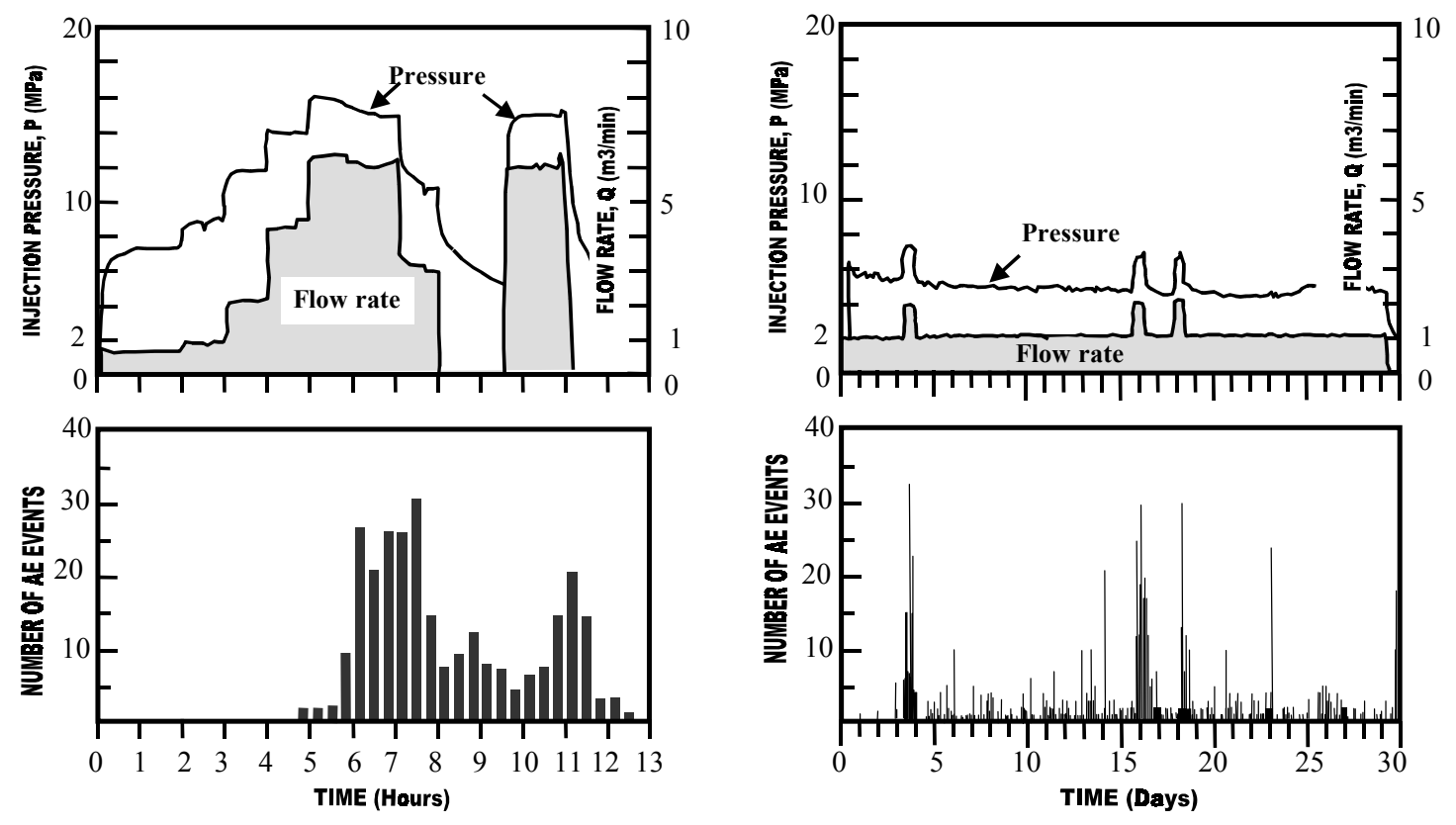

(a)

(b)

Figure 15. Injection flow rate, injection pressure and seismic activities during fluid injection at Hijiori Hot dry rock site in Japan. (a) A one day high pressure hydraulic fracturing operation and (b) a one month low pressure circulation experiment (modified from Sasaki 1998) 


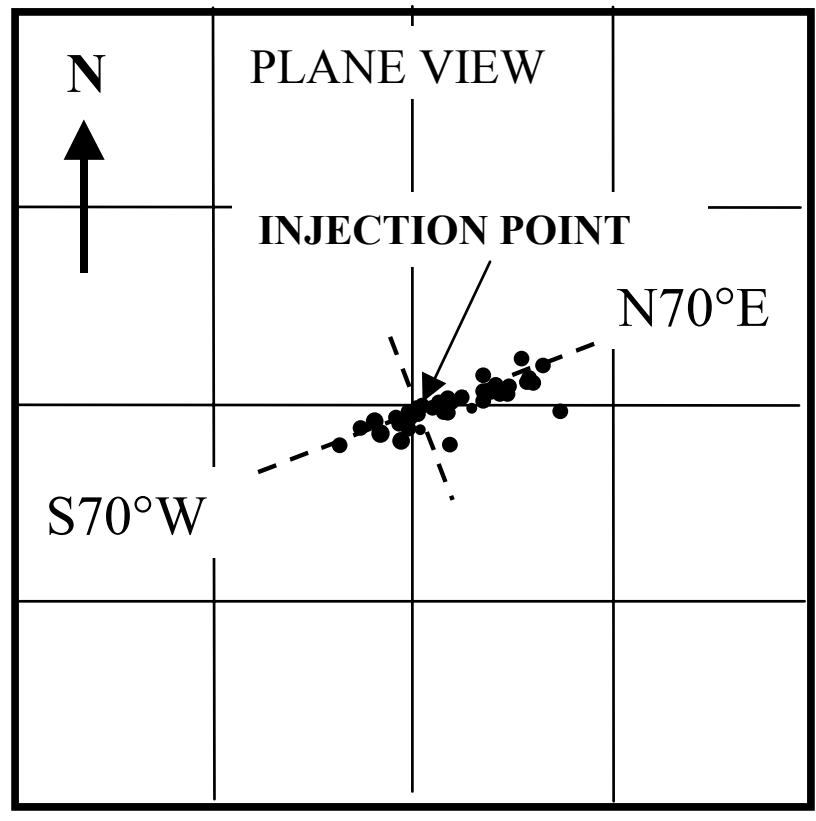

(a)

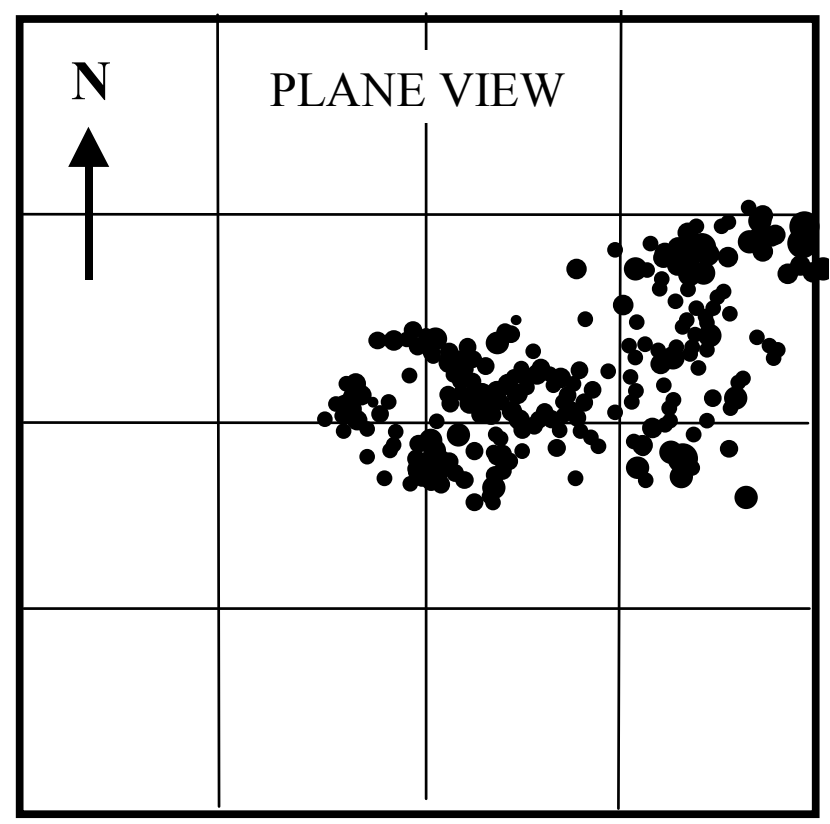

(b)

Figure 16. Spatial distribution of the micro-seismic events at Hijiori Hot dry rock site in Japan. (a) events during a one day high pressure hydraulic fracturing operation. (b) events during a one month low pressure circulation experiment (modified form Sasaki (1998)) 


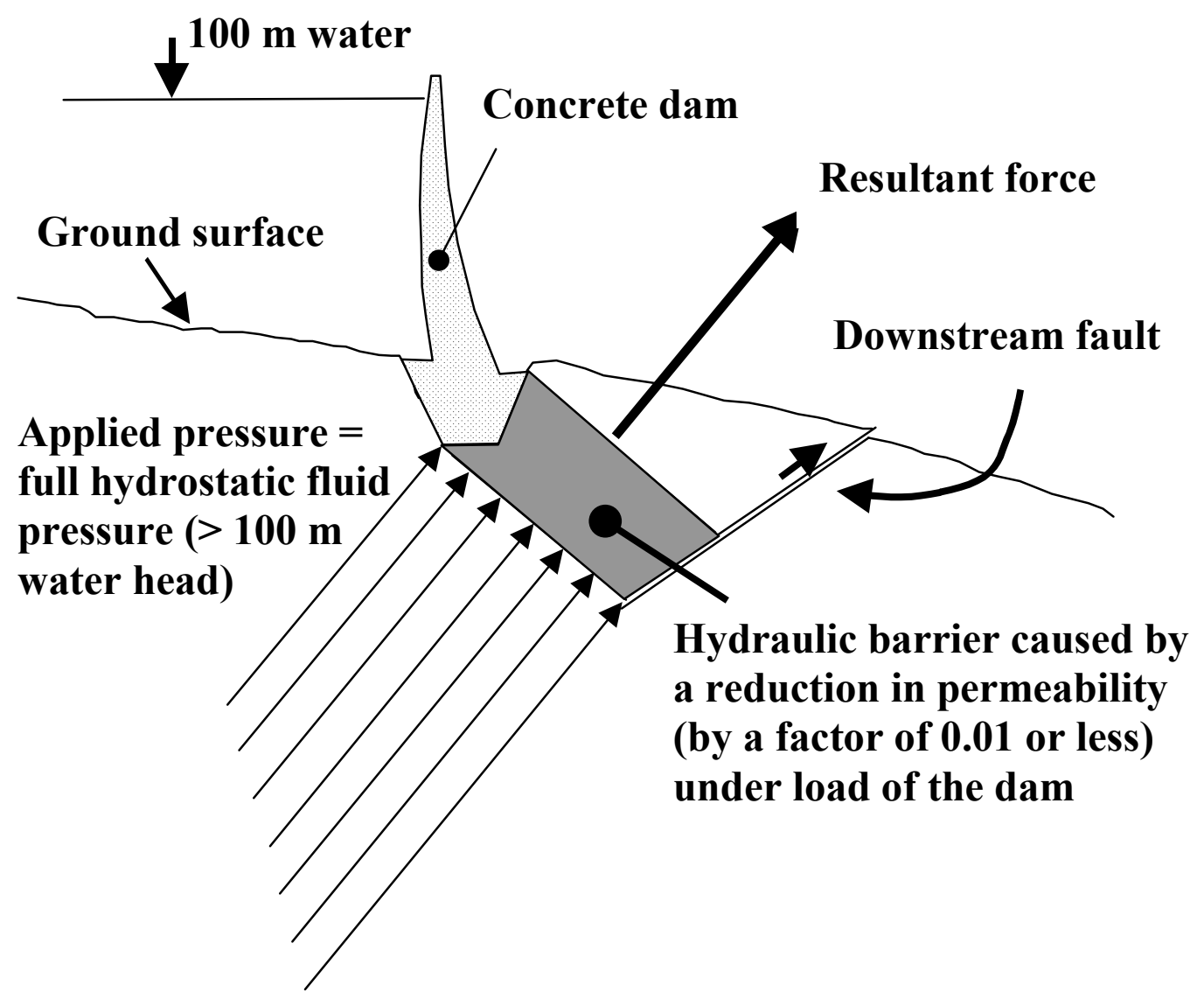

Figure 17. Londe's hypothesis for the 1959 failure at the Malpassat Dam, France. The figure shows how an underground hydraulic barrier was created under load of the dam due to extremely stress-sensitive permeability (modified from Londe (1987)) 


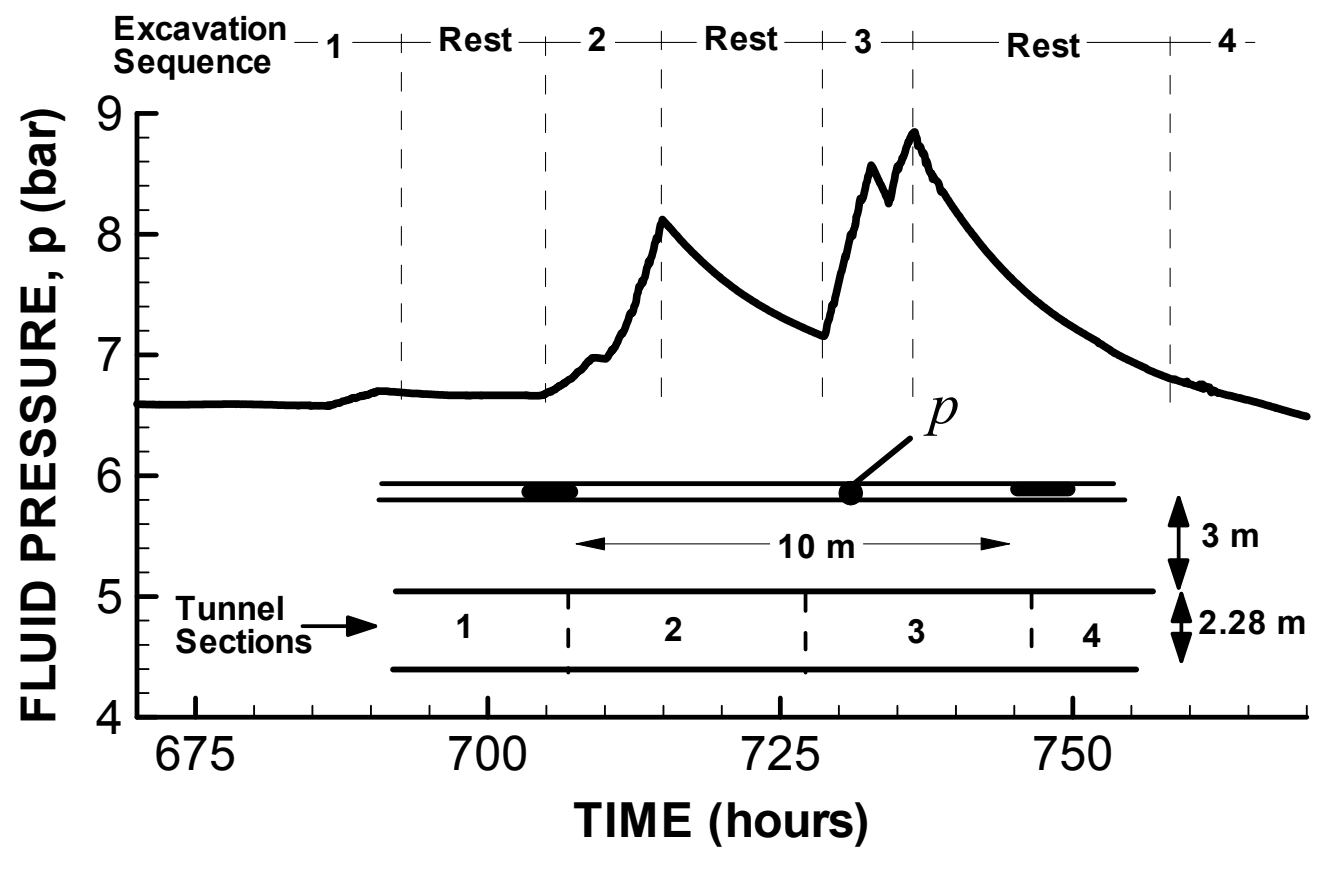

Figure 18. Excavation induced pressure changes in rocks during tunnel boring at the Grimsel Test Site in Switzerland. The pressure is measured in a 10 meter long packed-off section of a borehole located about 3 meters from the wall of the tunnel (see sketch of tunnel and monitoring section below the pressure curve) 


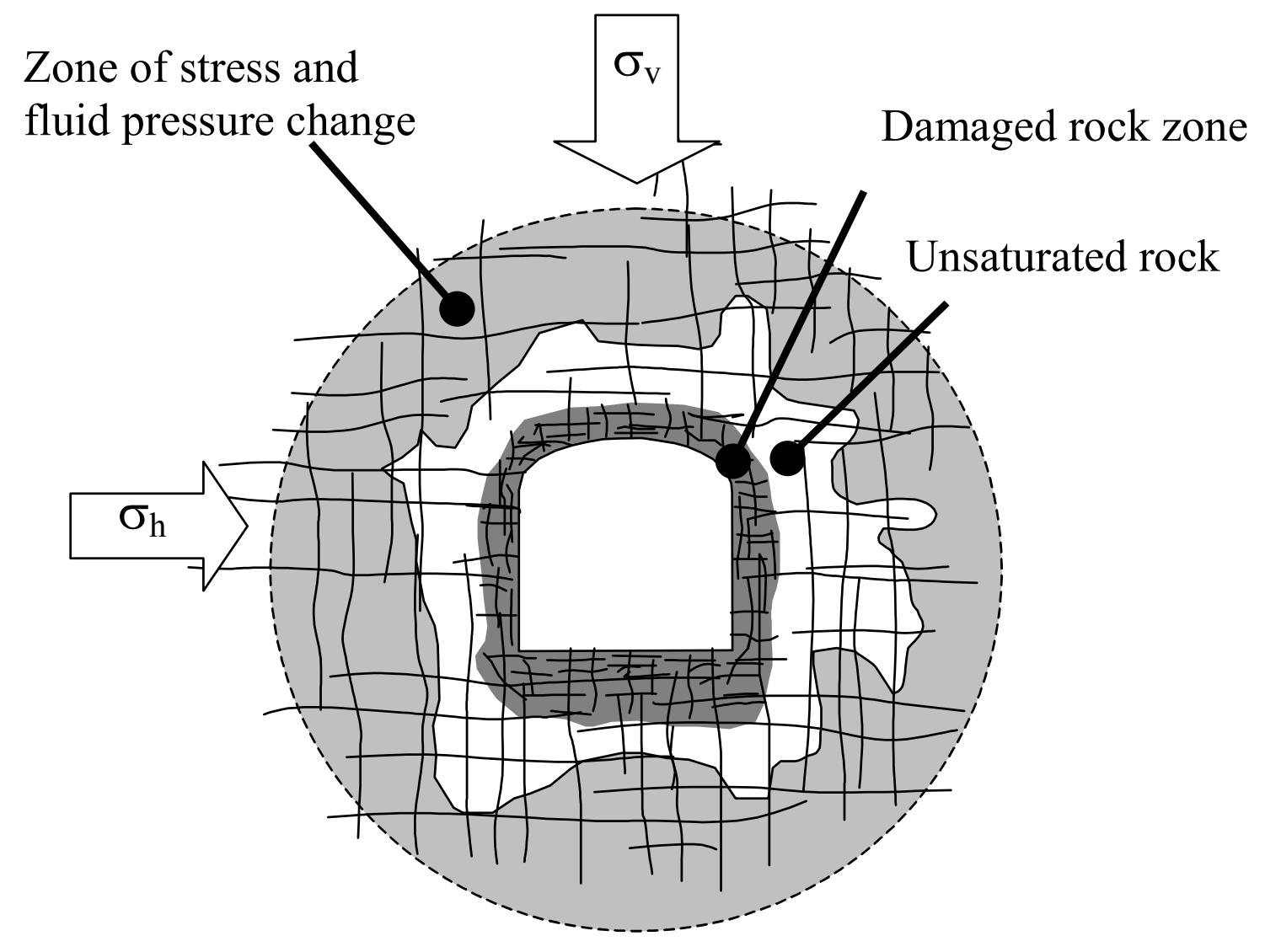

Figure 19. Schematic of the Excavation Disturbed Zone (EDZ) around a drift in fractured rock 


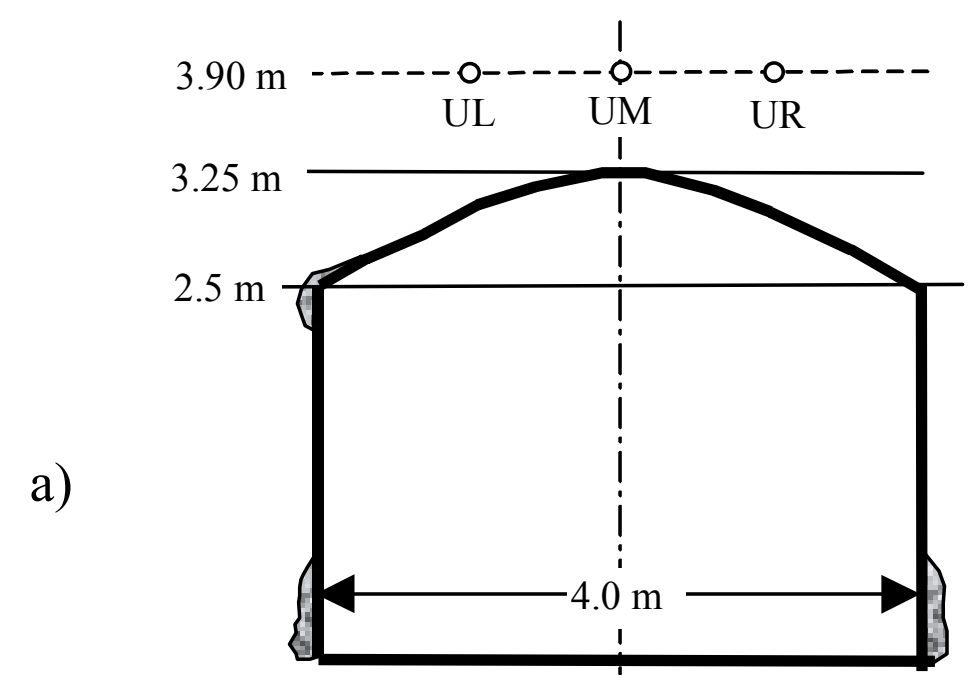

\section{$\bullet \mathrm{UL} \quad \mathrm{UM} \triangle \mathrm{UR}$}

b)

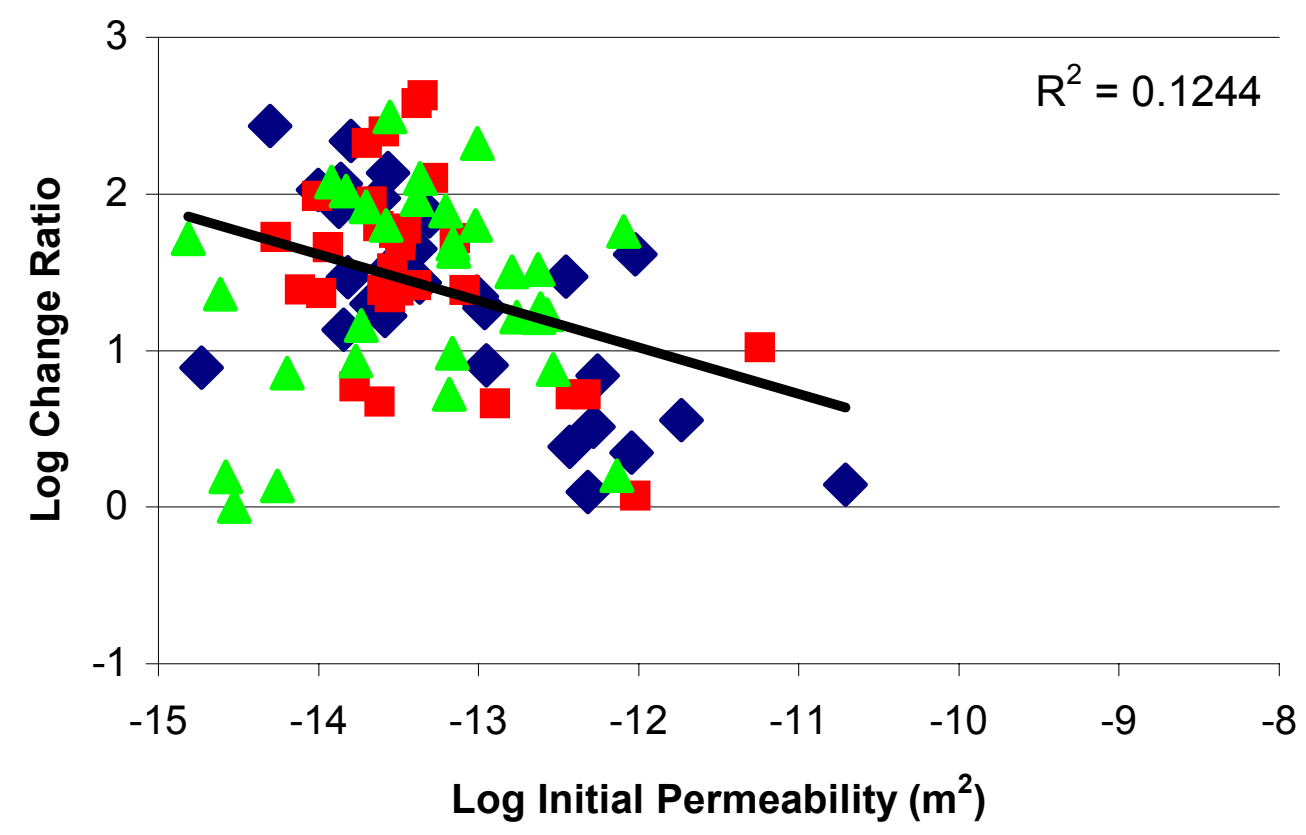

Figure 20. Pre- to post excavation air-permeability tests conducted in fractured unsaturated tuff at Yucca Mountain, Nevada. (a) Geometry of tunnel and test boreholes and (b) Ratio of Pre- and post excavation permeability plotted against pre-excavation permeability (from Wang et al 2002) 


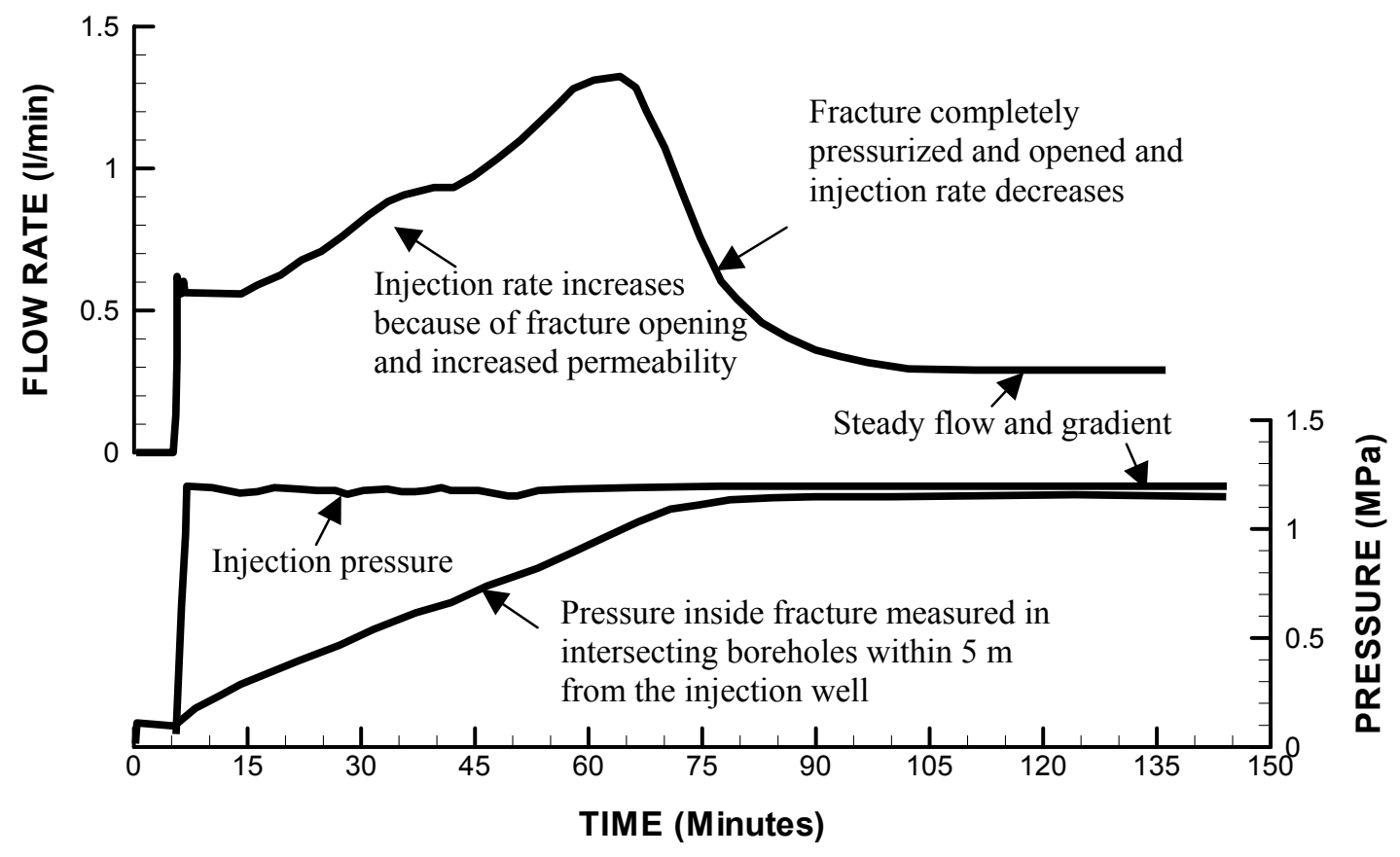

Figure 21. Flow and pressure response during a constant pressure injection into a subhorizontal fracture located at 70 meters depth. The injection pressure is $1.2 \mathrm{MPa}$ and the vertical stress normal to the fracture is estimated to $2.0 \mathrm{MPa}$. Note that the injection rate increases during the first 60 minutes despite a reducing pressure gradient. This can be explained by an increased permeability as a result of increased fluid pressure within the fracture (data from Alm 1999) 


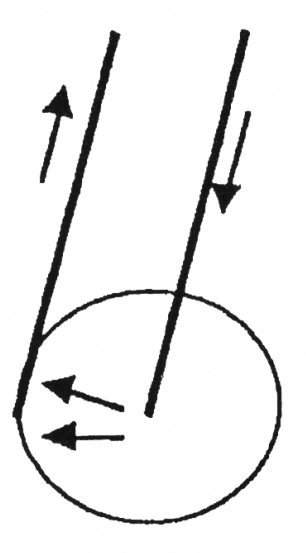

(a)

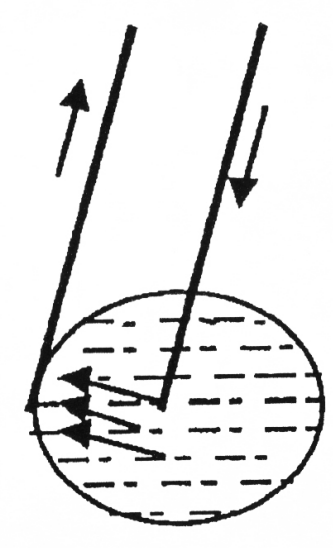

(b)

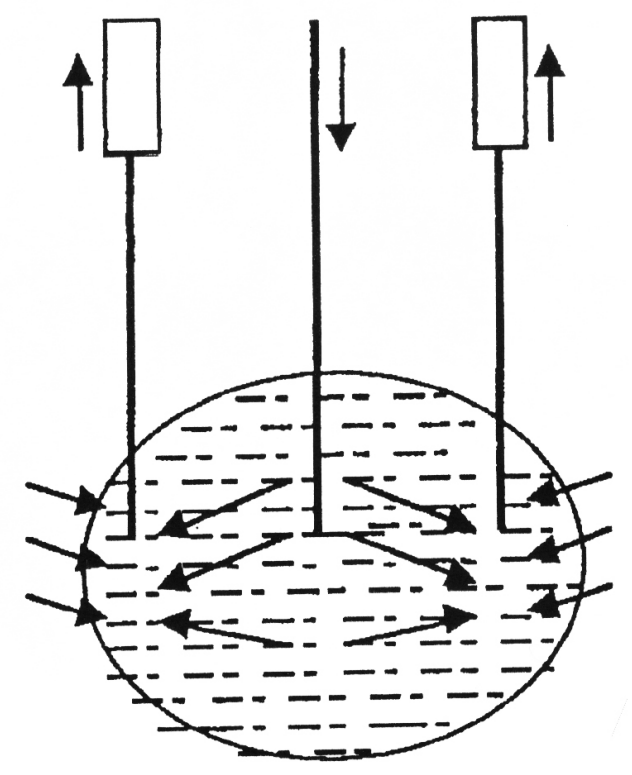

(c)

Figure 22. Development of the HDR/HWR reservoir concepts in the last 25 years. (a) Penny shaped fracture, (b) shear on natural joints and (c) graben concept. After Baria et al. (1999a) 


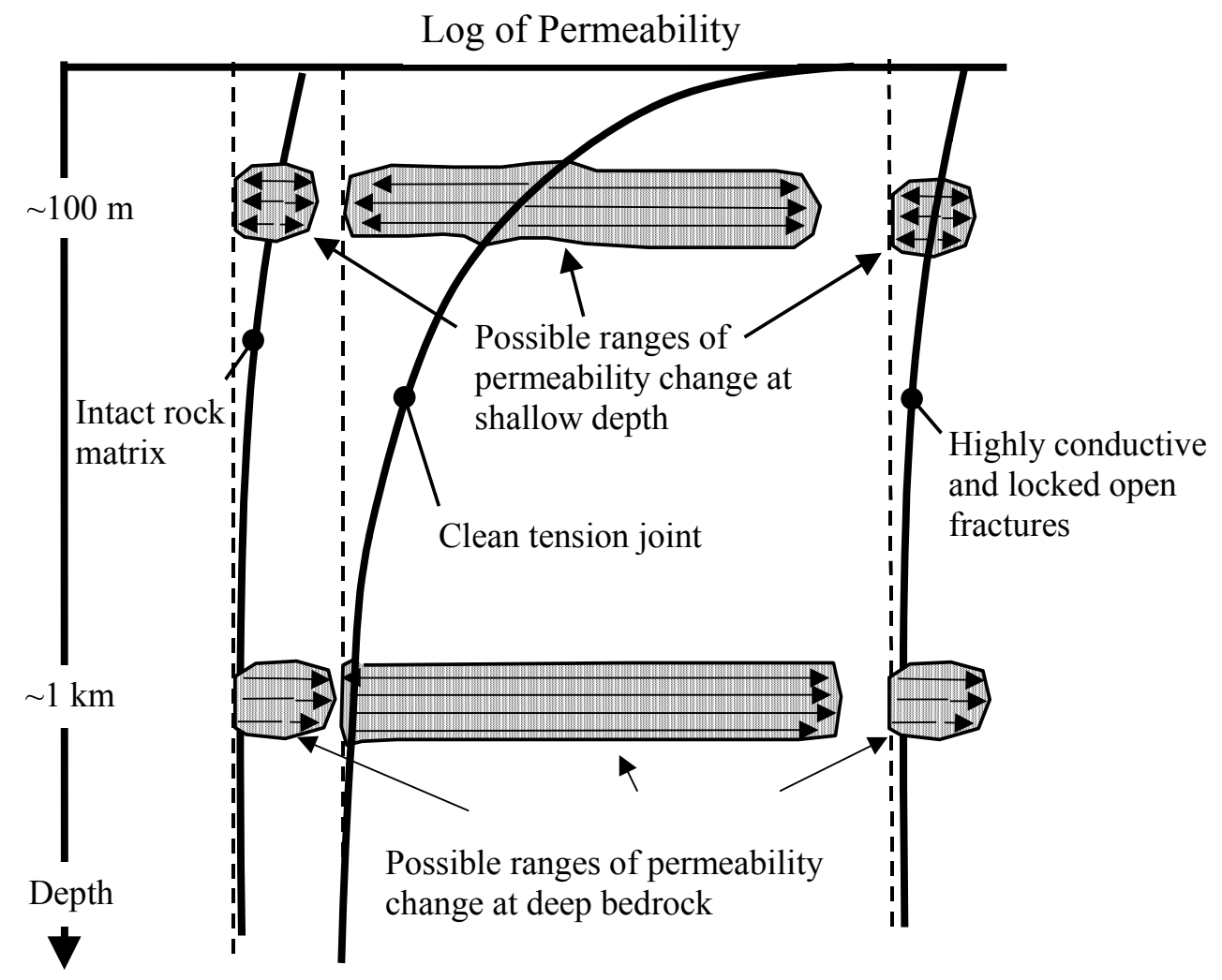

Figure 23. Schematic picture showing possible permeability changes at shallow and deep locations in fractured bedrock. The solid lines represent the depth- (or stress)permeability function for intact rock, clean tension joint and highly conductive and locked open fractures 